\title{
Investigation Into Use of Piezoelectric Sensors in a Wheeled Robot Tire For Surface Characterization
}

\author{
Elizabeth G. Armstrong \\ Thesis submitted to the Faculty of the \\ Virginia Polytechnic Institute and State University \\ in partial fulfillment of the requirements for the degree of
}

\author{
Master of Science \\ in \\ Mechanical Engineering
}

Corina Sandu, Chair

Saied Taheri

Mehdi Ahmadian

7 June, 2013

Blacksburg, Virginia

Keywords: intelligent tire, wheeled robot, terramechanics, piezoelectricity 


\title{
Investigation Into Use of Piezoelectric Sensors in a Wheeled Robot Tire For Surface Characterization
}

\author{
Elizabeth G. Armstrong
}

\section{(ABSTRACT)}

A differential steered, $13.6 \mathrm{~kg}$ robot was developed as an intelligent tire testing system and was used to investigate the potential of using piezoelectric film sensors in small tube-type pneumatic tires to characterize tire-ground interaction.

One focus of recent research in the tire industry has been on instrumenting tires with sensors to monitor the tire, vehicle, or external environment. On small robots, tire sensors that measure the forces and deflections in the contact patch could be used to improve energy efficiency and/or mobility during a mission.

The robot was assembled from a SuperDroid Robots kit and instrumented with low-cost piezoelectric film sensors from Measurement Specialties between the inner tube and the tire. An unlaminated and a laminated sensor were placed circumferentially along the tread and an unlaminated sensor was placed along the sidewall. A slip ring transferred the signals from the tire to the robot. There, the signal conditioning circuit extended the time constant of the sensors and filtered electromagnetic interference. The robot was tested with a controlled power sequence carried out on polished cement, ice, and sand at three power levels, two payload levels, and with two tire sizes.

The results suggest that the sensors were capable of detecting normal pressure, deflection, and/or longitudinal strain. Added payload increased the amplitude of the signals for all sensors. On the smaller tires, sensors generally recorded a smaller, wider signal on sand compared to cement, indicating the potential to detect contact patch pressure and length. The signals recorded by the unlaminated sensor along the tread of the smaller tire were lower on ice compared to cement, indicating possible sensitivity to tractive force. Results were less consistent for the larger tires, possibly due to the large tread pattern. 


\section{Acknowledgments}

I am grateful to Dr. Corina Sandu for her guidance and patience the past two years while serving as my advisor. She has been supportive of my academic and professional goals and has taken an interest in my success. I would like to thank Dr. Saied Taheri for developing the idea for this project and providing financial support for materials. An additional thank you to Dr. Mehdi Ahmadian for his welcoming, supportive approach to working with students and for serving on my committee.

The Mechanical Engineering Department at Virginia Tech provided me with a Graduate Teaching Assistantship for three semesters. I value this opportunity not only for the financial support received, but also for exposing me to the undergraduate engineering curriculum that I missed as a physics major. Furthermore, the teaching skills that I developed as a graduate teaching assistant will serve me well into the future.

The expectation in my family has long been that I should always 'do my best'. Throughout my life, my family has helped to reduce barriers that may stand in the way of me doing and achieving my best. For having my Mom, Dad, and sister Meghan in my life, I am grateful.

I am the product of an environment that values education, asking questions, and having thoughtful discussion. The exceptional teachers and strong math curriculum in the Ann Arbor Public Schools laid the foundation for me to succeed in college and graduate school. My undergraduate education at Wake Forest University taught me to approach new topics from the perspective of how they fit into the world. Throughout these experiences, I have been surrounded by loyal friends to challenge and support me. To the people and places that have surrounded me in my life to this point, thank you.

Finally, I would like to acknowledge three other graduate students for their direct assistance with this research project: Anudeep Bhoopalam for preparing and maintaining the ice test surface and taking the friction measurements; Rea Cauthen for patiently answering many mechanical questions while I was assembling the robot; and Thomas Ruscher for his helpful consultations regarding the signal conditioning circuit. 


\section{Contents}

Abstract $\quad$ ii

$\begin{array}{ll}\text { Acknowledgments } & \text { iii }\end{array}$

List of Figures $\quad$ vii

List of Tables $\quad$ ix

Nomenclature $\quad$ x

1 Introduction $\quad 1$

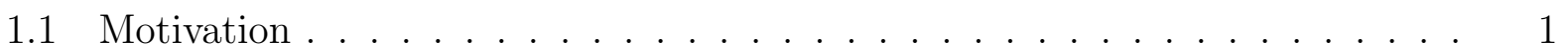

1.1.1 Intelligent Tire Systems . . . . . . . . . . . . . . . . . . . . 1

1.1.2 Small Tires and Robots . . . . . . . . . . . . . . . . . . 2

1.1.3 Applications of Intelligent Tire Technology for Small Tires . . . . . . 2

1.2 Study Objectives . . . . . . . . . . . . . . . . . . . . . . . . . . . . . . . .

1.3 Outline of Thesis . . . . . . . . . . . . . . . . 3

2 Literature Review $\quad 5$

2.1 Study of Tires . . . . . . . . . . . . . . . . . . . . . . . . . . . . . . . .

2.1.1 Tires on Hard Surfaces . . . . . . . . . . . . . . . . . . . . 6

2.1.2 Tires on Deformable Surfaces . . . . . . . . . . . . . . 7

2.2 Current State of the Art for Intelligent Tire Sensors . . . . . . . . . . . . 8

2.2.1 Sidewall Torsion Sensor . . . . . . . . . . . . . . . 8

2.2.2 Darmstadt Sensor . . . . . . . . . . . . . . . . . . 9

2.2.3 Surface Acoustic Wave Sensor . . . . . . . . . . . . . . . . . . 9

2.2.4 Piezoelectric Sensors . . . . . . . . . . . . . . . . . . . . . . . . . . . . . . . .

2.3 Review of Previous Related Studies . . . . . . . . . . . . . . . . . . . 11

2.3.1 Previous Studies Using a Wheeled Robot to Classify Terrain . . . . . 12

2.3.2 Previous Studies Using Piezoelectric Sensors in Tires . . . . . . . . . 12

3 Design of Test System 14

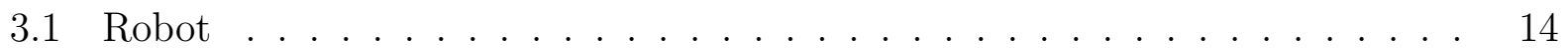

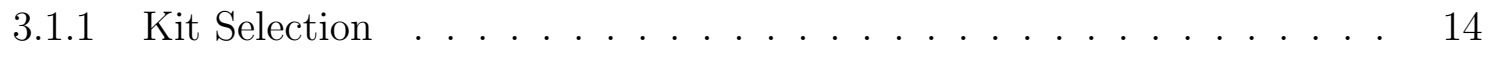


3.1 .2 Chassis . . . . . . . . . . . . . . . . . . . . . . . . . 18

3.1 .3 Motors and Encoders . . . . . . . . . . . . . . . . . . . . . . 18

3.1 .4 Motor Controller . . . . . . . . . . . . . . . . . . . . . . . . 21

3.1 .5 Wheels and Tires . . . . . . . . . . . . . . . . . . 22

3.2 Piezoelectric Sensors . . . . . . . . . . . . . . . . . . . . . . 26

3.2.1 Strains Experienced by Piezoelectric Sensors on a Tire . . . . . . . 26

3.2.2 Planned Placement of Piezoelectric Sensors . . . . . . . . . . . . . . 27

3.2.3 Investigation Into Possible Adhesives For Sensors Attachment . . . . 28

3.3 Data Transmission and Acquisition . . . . . . . . . . . . . . . 29

3.3 .1 Slip Rings . . . . . . . . . . . . . . . . . . . . . . . . 30

3.3.2 Data Acquisition Hardware . . . . . . . . . . . . . . . . . . . 32

3.3 .3 On-Board Computer . . . . . . . . . . . . . . . . . . . . . . 33

3.3.4 LabView Virtual Instrument . . . . . . . . . . . . . . . . . . . . . 33

3.4 Signal Conditioning . . . . . . . . . . . . . . . . . . . . . . . . 34

3.4.1 Design Constraints for Signal Conditioning System . . . . . . . . . 34

3.4.2 Identification and Reduction Electromagnetic Interference . . . . . . 35

3.4.3 Evaluation of Input Signal Strength and Frequency Range . . . . . . 37

3.4.4 Design of Signal Conditioning Circuit . . . . . . . . . . . . . . 38

3.4.5 Operational Amplifier Selection . . . . . . . . . . . . . . . . . . 43

3.4.6 Construction of Signal Conditioning Circuit . . . . . . . . . . . . 43

3.5 Review of Design of Test System . . . . . . . . . . . . . . . . . . . 44

4 Testing Methodology 46

4.1 Test Parameters . . . . . . . . . . . . . . . . . . . . . . . . . . 46

4.1 .1 Tire Size . . . . . . . . . . . . . . . . . . . . . 47

$4.1 .2 \quad$ Applied Power . . . . . . . . . . . . . . . . . . . . . . . 47

4.1 .3 Payload . . . . . . . . . . . . . . . . . . . . . . . . . . . 48

4.1 .4 Surfaces . . . . . . . . . . . . . . . . . . . . . . 50

4.1.5 Piezoelectric Sensors . . . . . . . . . . . . . . . . . . 50

4.2 Surface Preparation and Characterization . . . . . . . . . . . . . . . 52

4.2 .1 Hard Surface Preparation . . . . . . . . . . . . . . . . 52

4.2.2 Measurement of Static Coefficient of Friction of Hard Surfaces . . . . 52

4.2 .3 Sand Preparation . . . . . . . . . . . . . . . . . . . 52

4.2 .4 Measurement of Cone Index of Sand . . . . . . . . . . . . . . . 53

4.2 .5 Temperature of Tires . . . . . . . . . . . . . . . . . 53

4.3 Measurements Taken During Each Test . . . . . . . . . . . . . . . . . . 54

4.3.1 Motor Configuration Parameters From Motor Controller . . . . . . . 54

4.3.2 Speed and Power Levels From Motor Controller . . . . . . . . . . . 55

4.4 Data Processing . . . . . . . . . . . . . . . . . . . . . . 55

$4.4 .1 \quad$ Pre-Processing . . . . . . . . . . . . . . . . . . 56

4.4 .2 Separation of Wheel Rotations . . . . . . . . . . . . . . . 56

4.4 .3 Signal Characterization . . . . . . . . . . . . . . . . . 60 
5 Results and Discussion $\quad 63$

5.1 Surface Characterization . . . . . . . . . . . . . . . . . . . 63

5.1 .1 Coefficient of Static Friction for Hard Surfaces . . . . . . . . . . . 63

5.1 .2 Cone Index of Sand . . . . . . . . . . . . . . . . . . . . . . 63

5.1 .3 Temperature . . . . . . . . . . . . . . . . . . . 64

5.2 Measurements of the Robot . . . . . . . . . . . . . . . . . . 66

5.2 .1 Static Load on Each Tire . . . . . . . . . . . . . . . . . 66

5.2 .2 Power and Wheel Speed . . . . . . . . . . . . . . . . . . 66

5.3 Results from Piezoelectric Sensors . . . . . . . . . . . . . . . . . 70

5.3 .1 Sensor Placement . . . . . . . . . . . . . . . . . . . . 70

5.3.2 Qualitative Analysis of Measurements from Piezoelectric Sensors . . . 71

5.3 .3 Signal Amplitude . . . . . . . . . . . . . . . . . . . . . . 73

5.3 .4 Signal Width . . . . . . . . . . . . . . . . . . . . . . . 75

5.3 .5 Summary of Results . . . . . . . . . . . . . . . . . . 78

5.4 Limitations . . . . . . . . . . . . . . . . . . . . . . . . 80

6 Conclusion $\quad 81$

6.1 Evaluation of Progress Toward Objectives . . . . . . . . . . . . . 81

6.1 .1 Evaluation of Objective $1 \ldots \ldots \ldots$. . . . . . . . . . 81

6.1 .2 Evaluation of Objective $2 \ldots \ldots \ldots \ldots$. . . . . . . . . 81

6.1 .3 Evaluation of Objective $3 \ldots \ldots \ldots \ldots$

6.1 .4 Evaluation of Objective $4 \ldots \ldots \ldots$. . . . . . . . . . . . . 82

6.2 Recommendations for Future Work . . . . . . . . . . . . . . . 83

\section{Appendices}

$\begin{array}{lr}\text { A Connectors Used on Robot } & 86\end{array}$

$\begin{array}{ll}\text { B LabView Virtual Instrument } & 87\end{array}$

$\begin{array}{ll}\text { C MicroBasic Script for 6" Tires } & 89\end{array}$

D MicroBasic Script for 10" Tires 94

$\begin{array}{ll}\text { Bibliography } & 99\end{array}$ 


\section{List of Figures}

2.1 Dimensions and axes of a piezoelectric film sensor . . . . . . . . . . . . 10

3.1 Photograph of underside of Heavy Duty ATR chassis by SuperDroid. . . . . 19

3.2 Photograph of chain-sprocket mechanism connecting motors to wheel hubs. . 20

3.3 Photograph of a $24 \mathrm{~V}, 120$ RPM motor with attached two channel Hall effect

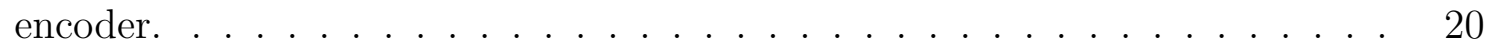

3.4 Photograph of Roboteq SDC2130 motor controller. . . . . . . . . . . . . . 22

3.5 Photographs of the two tire treads. . . . . . . . . . . . . . . . . 24

3.6 Photographs of the two tire sides with the 6" tire on the left and the 10" tire on the right. ............................ 25

3.7 Diagram of mounting locations and types of piezoelectric sensors used in testing. 28

3.8 Block diagram of the test system components and their relative connections. 30

3.9 Diagram of the tire sensor layout and connections to the on-board electronics. 31

3.10 Photograph of the slip ring mounted on the axle for the 10 " tires. . . . . . . 32

3.11 Circuit diagram of a charge amplifier, powered by a single supply op amp. $\quad$. 39

3.12 Circuit diagram of an inverting amplifier, powered by a single supply op amp. 40

3.13 Circuit diagram for the two stage signal conditioning circuit. . . . . . . . . . 41

3.14 Transfer function for signal conditioner, created in TINA-TI. . . . . . . . . . 42

3.15 Signal conditioner created on a prototype board, placed in an enclosure. . . . 44

4.1 Location of $10 \mathrm{lb}$ weight placed under the laptop computer. . . . . . . . . . 49

4.2 Photograph of robot on test surfaces . . . . . . . . . . . . . . . . 51

4.3 Tube system used to create and maintain ice testing surface. . . . . . . . . 53

4.4 Photographs of prepared sand . . . . . . . . . . . . . . . . . . . . 54

4.5 Graphs showing an example of conversion from speed vs time to speed vs angle. 58

4.6 Graphs showing an example of separation of angle rotations. . . . . . . . . . 59

4.7 Graphs illustrating the process of shifting the signal measured during each rotation of the wheel so that any slants and offsets are corrected. . . . . . . . 61

4.8 Graph demonstrating the full width at half maximum method to characterize the width of the signal for each rotation. . . . . . . . . . . . . . 62

5.1 Cone index measurements for sand on the second day (6" tires) of testing. . 64

5.2 Representative curves, taken from $10 \mathrm{lb}$ payload, $100 \%$ power, on cement. . . 72 
5.3 Bar graph of average maximum amplitude for sensor 1 (unlaminated, circumferential) for each test condition. . . . . . . . . . . . . . . . . 74

5.4 Bar graph of average maximum amplitude for sensor 2 (unlaminated, radial) for each test condition for the 6 " tires. . . . . . . . . . . . . . . . 75

5.5 Bar graph of average maximum amplitude for sensor 3 (laminated, circumferential) for each test condition. . . . . . . . . . . . . . . . 76

5.6 Bar graph of average sensor 1 FWHM for each test condition. . . . . . . . . 77

5.7 Bar graph of average sensor 2 FWHM for each test condition on 6" tires. . . 78

5.8 Bar graph of average sensor 3 FWHM for each test condition. . . . . . . . . 79

B.1 Screenshot of the front panel of the LabView VI developed for this test. . . . 88 


\section{List of Tables}

2.1 Physical and electrical characteristics of PVDF piezoelectric sensors. . . . . . 10

3.1 Criteria considered during selection of a robot or robot kit. . . . . . . . . 16

3.2 Comparison of capabilities of typical hobby cars, the SuperDroid Heavy Duty ATR and the GEARS SMP. . . . . . . . . . . . . . . . . 17

3.3 Specifications for the IG42 $24 \mathrm{~V}$ motor. . . . . . . . . . . . . . . . . 18

3.4 Description of the two pneumatic tires used in this study. The tires were acquired in the kit from SuperDroid. . . . . . . . . . . . . . . 23

3.5 Piezoelectric sensors considered from Measurement Specialties. . . . . . . . . 26

3.6 Specifications for the SRA-73683-6 slip ring. . . . . . . . . . . . . . . . 31

3.7 Measurement Computing PMD-1208LS DAQ connections. . . . . . . . . . . 33

3.8 Possible noise sources to identify how to reduce them and what frequencies the conditioner should attenuate. . . . . . . . . . . . . . 36

4.1 Details of the programmed power sequences for each tire size and applied power. 48

4.2 Piezoelectric sensors and their locations used in testing . . . . . . . . . . . 50

5.1 Range of temperature measurements for top each tire size . . . . . . . . . 65

5.2 Weight distribution across four wheels of the robot, based on tire and payload configuration. . . . . . . . . . . . . . . . . 67

5.3 Speed and power for $6 "$ tires . . . . . . . . . . . . . . . . . 67

5.4 Speed and power for 10" tires . . . . . . . . . . . . . . . . . 68

5.5 Angular differences in location between the piezoelectric sensors. . . . . . . . 70

A.1 Connectors added to the robot test system. . . . . . . . . . . 86 


\section{Nomenclature}

$\frac{\epsilon}{\epsilon_{0}} \quad$ Dielectric constant where $\epsilon_{0}=8.85 \frac{\mathrm{pF}}{\mathrm{m}}$

$\mu \quad$ Coefficient of friction

$\mu_{p} \quad$ Peak coefficient of friction

$\mu_{\text {sliding }}$ Sliding coefficient of friction

$\mu_{\text {static }}$ Static coefficient of friction

$\omega \quad$ Angular velocity of tire $\left[\frac{\mathrm{rad}}{\mathrm{s}}\right]$

$\omega_{\max }$ Maximum specified motor speed $[\mathrm{RPM}]$

$\omega_{\text {rel }} \quad$ Relative motor speed, measured by the motor controller $\left[\% \omega_{\max } \times 10\right]$

$\sigma_{m} \quad$ Applied stress, $6 \times 1$ vector $\left[\frac{\mathrm{N}}{\mathrm{m}^{2}}\right]$

$\tau \quad$ Time constant $[\mathrm{s}]$

$\theta \quad$ Angle [rad]

$\theta_{12}$ Angle by which sensor 2 leads sensor 1 [rad]

$\theta_{13}$ Angle by which sensor 3 leads sensor 1 [rad]

$A \quad$ Gain $\left[\frac{\mathrm{V}}{\mathrm{V}}\right]$ or $\left[\frac{\mathrm{V}}{\mathrm{C}}\right]$

C Capacitance $[\mathrm{F}]$

$C_{p} \quad$ Capacitance of a piezoelectric sensor $[\mathrm{F}]$

$D_{i} \quad$ Dielectric displacement, $3 \times 1$ vector $\left[\frac{\mathrm{C}}{\mathrm{m}^{2}}\right]$

$d_{i j} \quad$ Piezoelectric strain constant, $3 \times 6$ matrix $\left[\frac{\mathrm{C}}{\mathrm{N}}\right]=\left[\frac{\mathrm{m}}{\mathrm{V}}\right]$

$e_{i j} \quad$ Dielectric permittivity, $3 \times 3$ matrix $\left[\frac{\mathrm{F}}{\mathrm{m}}\right]$

$f \quad$ Frequency $[\mathrm{f}]$ 
$F_{x} \quad$ Force in the x direction, longitudinal or tractive force $[\mathrm{N}]$

$F_{z} \quad$ Force in the $\mathrm{z}$ direction, vertical load $[\mathrm{N}]$

$I_{\max } \quad$ Maximum allowable current for a motor channel $[\mathrm{A}]$

$I_{m o t}$ Current from one motor channel, measured by motor controller [A]

$l_{f} \quad$ Length of piezoelectric sensor protective film [m]

$P \quad$ Applied relative power level for a motor channel $\left[\% P_{\max } \times 10\right]$

$p \quad$ Pyroelectric coefficient $\left[\frac{\mathrm{C}}{\mathrm{m}^{2}} \mathrm{~K}\right]$

$P_{\max }$ Maximum theoretical power level for a motor channel $[\mathrm{A}]$

$P_{m o t} \quad$ Measured power level for a motor channel [A]

$P_{r e l} \quad$ Measured relative power level for a motor channel $\left[\% P_{\max } \times 10\right]$

$q \quad$ Charge $[\mathrm{C}]$

$R \quad$ Resistance $[\Omega]$

$R_{p} \quad$ Resistance of a piezoelectric sensor $[\Omega]$

$r_{\text {eff }} \quad$ Effective rolling radius of tire $[\mathrm{m}]$

$s \quad$ Slip

$T \quad$ Absolute temperature $[\mathrm{K}]$

$t_{d a q}$ Set of evenly spaced time values recorded corresponding to the DAQ measurements $[\mathrm{s}]$

$t_{m o t} \quad$ Set of time values recorded corresponding to motor measurements [ms]

$v \quad$ Linear velocity of vehicle $\left[\frac{\mathrm{m}}{\mathrm{s}}\right]$

$V_{0} \quad$ Initial voltage $[\mathrm{V}]$

$V_{p} \quad$ Voltage measured across a piezoelectric sensor $[\mathrm{V}]$

$V_{\text {batt }} \quad$ Battery voltage $[\mathrm{V}]$

$W_{\text {front }}^{*}$ Adjusted weight of the front of the robot $[\mathrm{kg}]$

$W_{\text {left }}^{*}$ Adjusted weight of the left side of the robot $[\mathrm{kg}]$

$W_{\text {rear }}^{*}$ Adjusted weight of the rear of the robot $[\mathrm{kg}]$ 
$W_{\text {right }}^{*}$ Adjusted weight of the right side of the robot $[\mathrm{kg}]$

$w_{f} \quad$ Width of piezoelectric sensor protective film $[\mathrm{m}]$

$W_{F L} \quad$ Calculated front left weight of the robot $[\mathrm{kg}]$

$W_{\text {front }}$ Measured weight of the front of the robot $[\mathrm{kg}]$

$W_{F R}$ Calculated front right weight of the robot $[\mathrm{kg}]$

$W_{\text {left }}$ Measured weight of the left side of the robot [kg]

$W_{\text {rear }}$ Measured weight of the rear of the robot [kg]

$W_{\text {right }}$ Measured weight of the right side of the robot $[\mathrm{kg}]$

$W_{R L} \quad$ Calculated rear left weight of the robot $[\mathrm{kg}]$

$W_{R R} \quad$ Calculated rear right weight of the robot $[\mathrm{kg}]$

$W_{\text {total }}$ Measured total weight of the robot $[\mathrm{kg}]$

$Z \quad$ Electrical impedance $[\Omega]$

$Z_{C} \quad$ Electrical impedance of a capacitor $[\Omega]$

$Z_{R} \quad$ Electrical impedance of a resistor $[\Omega]$

AVDL Advanced Vehicle Dynamics Laboratory, Virginia Tech

CI Cone index $[\mathrm{kPa}]$

CMS Computational Multi-Physics Systems Laboratory, Virginia Tech

CVeSS Center for Vehicle Systems and Safety, Virginia Tech

FWHM Full Width at Half Maximum

GRR Gear reduction ratio

PPR Pulses per revolution

PVDF Polyvinylidene fluoride 


\section{Chapter 1}

\section{Introduction}

In this chapter, the motivation for the present study and the specific study objectives are presented, followed by an outline of this document.

\subsection{Motivation}

This study combines research in the areas of intelligent tire systems and robotics. The goals of intelligent tire systems and a summary of uses for small wheeled mobile robots are presented in this section. Applications for implementing an intelligent tire system in a small wheeled mobile robot are presented.

\subsubsection{Intelligent Tire Systems}

In the past two decades, a focus of tire research has been on implementing sensors into tires to gather information about the tires, the vehicle, or the operating environment $[2,19]$. Tuononen [70] categorized the types of information that can be gathered from tire sensors as:

- Tire pressure

- Forces and moments acting on the tire

- Slip ratio and angle

- Tire wear

- Suspension wear

This information can be useful for both the driver and for the systems of the vehicle, such as those that improve vehicle traction or stability. Tires with embedded sensors are known as 'smart' or 'intelligent' tires. 


\subsubsection{Small Tires and Robots}

While a large amount of research has been done on development of 'smart' car tires, less work has been conducted on instrumentation of small tires, like those that are used in small wheeled robots. Small wheeled robots have uses across many fields in which tire sensors may have the potential to be useful.

In the military, wheeled robots can be used to explore hazardous terrain or areas that are unsafe for humans. The size of small robots allows them to explore areas that may be too confined for larger vehicles. In agricultural applications, it is important that vehicles traversing a terrain do not affect the properties of the terrain itself. Small robots are advantageous in this regard because they can be lightweight and designed to not damage the underlying terrain. This feature can also be useful for covert exploration. Robots have also been used for extraterrestrial applications, like the Mars rover [13, 39].

In many of the applications for small robots, the robot is used on deformable terrain like sand or grass rather than hard terrain like cement and asphalt. The tire-ground interaction is different on hard surfaces compared to soft surfaces and is therefore a subject of interest for many of the uses for robots discussed above. In the following section, possible applications for implementing sensors into the tires of small robotic tires are discussed.

\subsubsection{Applications of Intelligent Tire Technology for Small Tires}

Instrumenting tires of small wheeled robots with sensors to detect the forces or moments acting on a tire has potential to both improve robot mobility and enable the robot to sense its external environment. Sensors in a small robotic tire could be used to detect:

- Terrain type (ex. terrain roughness, properties of soil)

- Geometry of terrain and any obstacles

- Contact patch pressure [56]

- Tire sinkage

This type of information gathered by 'smart' robot tires could be used to:

- Improve traction, handling, and steering [11, 25]

- Improve vehicle durability for military missions [50]

- Develop terrain mobility map to plan the best path for future missions [71]

- Modify a mission plan to

minimize power consumption [25]

reduce terrain compaction

avoid unexpected immobilization [27] 
Study of small tires may also offer insights into how the properties of tires scale between different sizes. Many theoretical tire models produce results consistent with experimental data for car tires but not for tires of much larger size like those in agriculture and mining. Attempts to develop and validate models for these very large tires are complicated by logistical challenges caused by their size. Small tires on the other hand are inexpensive and simple to test. Instrumenting small tires with sensors may lead to improved understanding of the kinematics and dynamics of these tires compared to car tires. By evaluating the scaling ability of various car tire models relative the small robot tires, a scaling model has the potential to be extended to apply to larger tires that are difficult to study directly.

There is a great deal that can be gained by instrumenting tires on small wheeled robots with sensors capable of characterizing the tire-ground interaction. The measurements for these sensors can be used across a variety of fields to improve robot missions or assist in model development.

\section{$1.2 \quad$ Study Objectives}

The four objectives of the study presented in this document are:

1. Develop a low cost small wheeled mobile robot for intelligent tire testing.

2. Instrument the tires of the robot with sensors to characterize the tire-ground interaction.

3. Develop and implement a test procedure for using the robot with the sensors on off-road surfaces.

4. Investigate the possible information that can be gathered from these sensors regarding the tire-ground interaction.

The long term vision for this project is to implement the tires measurements into a control system that will improve robot mobility.

\subsection{Outline of Thesis}

Chapter 2 introduces the fundamental concepts in the study of tires on both hard and deformable surfaces. Existing tire sensor technology is presented, including an introduction to piezoelectric sensors like those used in this study. A review of related previous research is conducted. This research is fit into two categories: (1) using small wheeled mobile robots to characterize terrain and (2) using piezoelectric films as sensors in tires.

An in depth analysis of the design of the robot is presented Chapter 3. The selection of the small wheeled robot kit is presented, followed by information about the piezoelectric sensors used in the study. To obtain useful information from the sensors, a method to 
transfer a signal from the sensor in the tire to the computer on the robot using slip rings was developed. The signal conditioning circuit used with the piezoelectric sensors is explained.

In Chapter 4, the methodology used for testing the robot and piezoelectric sensors is presented. Five test parameters are examined: tire size, applied power, payload, surface type, and sensor location and type. The testing process is explained, starting with surface preparation and characterization, followed by a description of the measurements taken during each test. The steps of the data processing and analysis are then outlined.

In Chapter 5, the results from the tests are presented. The measurements taken to characterize the surfaces are provided along with the measured speed, power, and load. The processed signals from the piezoelectric sensors are presented and discussed. Limitations to the present study are then discussed.

Chapter 6 provides a conclusion to the study. Here, the progress toward each objective is addressed and suggestions are made for future studies. 


\section{Chapter 2}

\section{Literature Review}

This chapter begins with a general introduction to the study of tires including slip and different forms of motion resistance. The influence of parameters such as load, inflation pressure, and velocity on the tire-ground contact patch is reviewed for hard and soft surfaces. In Section 2.2, current tire sensors are introduced and in Section 2.3, previous similar studies are discussed.

\subsection{Study of Tires}

In this study, the three dimensional stress and deformation of the tire tread and sidewall during motion are of interest. In order to understand these stresses and deformation, it is important to consider how the energy put into a tire through applied torque is transferred to vehicle motion.

When a torque is applied to the tire, a tractive force is generated at the tire-ground contact patch. This force propels the vehicle forward. In an ideal scenario, all of the input energy would be transferred to vehicle motion. Losses of energy, however, can occur if there is inadequate friction available at the tire-ground interface, resulting in slip. Energy is also lost through various forms of motion resistance. Examples of motion resistance include aerodynamic losses, hysteresis of the tire, and resistance due to deformable terrains. This energy transfer can be summarized as

$$
\text { input energy - slip energy - motion resistance = output energy. }
$$

After all energy losses are considered, the resulting output energy is used to move the vehicle. This output energy can be measured by drawbar pull [75].

As previously stated, wheel slip occurs at the tire-ground contact patch and is dependent on the amount of friction available at the interface. The slip $s$ of a wheel moving at an angular velocity $\omega$ on a vehicle moving with a linear velocity $v$ can be defined as

$$
s=\frac{r_{e f f} \omega-v}{v}
$$


where $r_{\text {eff }}$ is the effective rolling radius of the tire $[24,28,71]$.

Changes can be made to tires to minimize the various forms of energy loss. Unfortunately, changes that reduce slip will often lead to increases in motion resistance and increases in wear. An example of this relationship is the type of rubber used on tires. Trucks tires are typically composed of natural rubber which provides increased durability. The drawback to natural rubber is that it provides less friction to the road. Car tires on the other hand are typically made of synthetic rubber. Synthetic rubber has better road adhesion but is less durable [71].

Throughout this paper, the focus is only on pneumatic tires. The dynamics of rigid wheels are not considered. Pneumatic tires are air-filled and are used for cars and trucks. The kinematics and dynamics of a pneumatic tires can very depending on the size, materials, manufacturing techniques, structure, and other parameters.

There are two primary methods used for sealing the air in the tire. On car and truck tires, a 'bead' is embedded in the rubber to seal the wheel to the rim. To prevent air loss through the rubber of the tire, a thin layer of rubber that is impermeable to air lines the inside of the tire [71]. A second method for sealing air is commonly used in bicycle tires and is also the method used for the tires in this study. In this method, a rubber tube is placed inside the tire that holds the air.

In the following sections, differences in the forces and deflections for pneumatic tires on hard and deformable surfaces are discussed.

\subsubsection{Tires on Hard Surfaces}

The ability of a rotating tire to generate vehicle motion depends on the ability to generate a tractive force $F_{x}$ at the tire-ground interface. The maximum or peak coefficient of friction available from a certain tire-ground combination is $\mu_{p}$.

Several theoretical models exist to describe the relationship between wheel slip and $F_{x}$. In all of the models, $F_{x}$ initially increases with increasing slip before reaching a maximum value around $15-20 \%$ slip. At higher levels of slip, $F_{x}$ decreases and levels off to a relatively constant value.

One of these theoretical models developed by Wong is presented here [71]. For a surface with maximum coefficient of friction $\mu_{p}$, the maximum theoretical longitudinal or tractive force on the tire at the tire-ground interface is

$$
F_{\text {xpeak }}=\mu_{p} F_{z}
$$

Tires will typically begin to experience slip before reaching this value. In the theory presented in Wong [71], the relationship between $F_{x}$ and slip for small levels of slip can be approximated as

$$
F_{x}=C_{i} s
$$

where $C_{i}$ is a characteristic of the tire. It is important to note here that the amount of slip experienced at a given $F_{x}$ is not dependent on $F_{z}$ at small slip values. 
During motion, the tire contact patch has two regions: a 'slip' (or 'sliding') region and a 'stick' (or 'static') region. The maximum longitudinal strain occurs at the boundary of the slip and stick regions and is directly proportional to the slip [28, 75]. At low slip, this boundary is located toward the front of the contact patch. For most driving on dry rigid surfaces, the amount of slip is typically small, less than 0.1 [28]. During driving, the strain occurs such that the outside of the tread is deflected in the direction of motion.

As $F_{x}$ increases with applied torque, it will reach a critical level, $F_{x c r i t i c a l}$ where

$$
F_{\text {xcritical }}=\frac{1}{2} \mu_{p} F_{z} .
$$

As $F_{x}$ increases, the boundary between the slip and stick regions moves rearward. In the theory presented in Wong [71], the relationship between $F_{x}$ and slip values of $F_{x}$ larger than $F_{x c r i t i c a l}$ but less than $F_{x p e a k}$ is

$$
F_{x}=\mu_{p} F_{z}\left(1-\frac{\mu_{p} F_{z}}{4 C_{i} s}\right) .
$$

This relationship is such that $F_{x}$ will continue to increase with slip, but at a lower rate until $F_{\text {xpeak }}$ is reached. Beyond $F_{\text {xpeak }}, F_{x}$ falls to

$$
F_{x s l i d i n g}=\mu_{s} F_{z}
$$

where $\mu_{s}$ is the sliding coefficient of friction.

The theory described above reveals some insights into the complicated relationship between $F_{x}, F_{z}, \mu_{p}$ and $s$. At larger $F_{z}$ or at larger $\mu_{p}, F_{x p e a k}$ is higher.

The peak friction available at the tire-ground interface depends not only on the surface, but also on the tire and operating conditions. Some tire designs and materials will have better adhesion to the road surface. Additionally, as speed increases, peak tire-road friction typically decreases, however the strength of this relationship depends on the properties of the tire [71].

Due to its relationship to rolling resistance, the deflection of the tire tread is also a parameter of interest in the study of tires. Deflection can be defined as the difference between the deformed and undeformed radii of a tire [32]. The deflection of a given tire depends greatly on the load and inflation pressure. As inflation pressure increases, tire deformation decreases. On rigid surfaces, as load increases, deformation increases [71]. These relationships can be different on deformable surfaces.

\subsubsection{Tires on Deformable Surfaces}

In the study of tires on rigid surfaces, the emphasis is primarily on the effects of the ground on the tire rather than the effects of the tire on the ground. In the study of tires on deformable surfaces, some focus is also directed toward the effect that tires have on the ground. For example, researchers with agricultural interests may be concerned about the volume of compacted soil and level of soil compaction caused by a tire. 
The stress distribution along the contact patch is more uniform on deformable surfaces compared to hard surfaces [75]. Like on rigid surfaces, the characteristics of the stresses in the tire-ground contact patch for a deformable surface are affected by the normal load on the tire and the inflation pressure. The average contact pressure typically increases with increases in wheel load [71]. This was seen in a study by Raper and Bailey [52] on radial tractor tires on agriculture surfaces. It was found that as the dynamic load was increased, in general, the soil-tire interface stresses increased and concentrated closer to the center of the contact patch. The same changes were seen for increases in inflation pressure.

The deformations at the tire-ground interface are dependent on the load on the tire. As load on a tire increases, the deformation of the tire increases [43] which leads to a larger tire-ground contact area and contact length $[52,58,71]$. The result on the deformable soil is a larger rut width and larger cross sectional area of deformed soil [52].

We have seen above that increasing load on a pneumatic tire on deformable surfaces can increase tire deformation. Decreasing inflation pressure of a pneumatic tire has a similar effect. Smaller inflation pressures will lead to larger contact area, contact length, and rut width $[43,52,58]$.

\subsection{Current State of the Art for Intelligent Tire Sen- sors}

The focus of this review will be on tire sensors that can measure forces or moments. During traction and braking, longitudinal forces on the tire are of interest. During turning maneuvers, lateral forces are of interest. Together, the longitudinal, lateral, and vertical forces can indicate the amount of unused friction available at the tire-ground interface [1] and has potential to provide information about the surface itself.

In 2002, a multi-year project was undertaken in the European Union to advance intelligent tire systems. In the published report for Deliverable D7, "Intelligent Tire Systems - State of the Art and Potential Technologies" [1] an overview of tire sensing technologies available at the time were provided. Those technologies are summarized in the following sections, along with more recent developments.

\subsubsection{Sidewall Torsion Sensor}

The Sidewall Torsion Sensor (SWT) was developed at Continental Tire in 1999 to measure the longitudinal and lateral forces and the torque acting on the tire [1,21]. This system uses a series of magnets embedded in the sidewall of the tire that are in alternating polarity. A sensor mounted to the car sensors the magnets as the tire rotates. A neural network system processes the data to predict forces and toque. The SWT is proprietary. 


\subsubsection{Darmstadt Sensor}

The Darmstadt Tire Sensor was developed by researchers at the University of Darmstadt in Germany $[1,2,15,74]$. Like the SWT, the Darmstadt sensor uses magnets embedded into the tire, but into the tread rather than the sidewall. The changes in the magnetic field are detected by a series of wires also embedded in the tread. These wires act as Hall effect sensors that register changes in signal as the tire deforms. The post-processing is based on the brush model of a tire. While useful for some applications, this sensor is likely too large for implementing in small robotic tires.

\subsubsection{Surface Acoustic Wave Sensor}

Surface Acoustic Wave (SAW) sensors can be used in many different ways to to monitor a tire $[1,51]$. These sensors consist of a small piece of metal on a piezoelectric film. The sensor measures electromagnetic waves and reflections. The limitations of these sensors are due to their durability in harsh conditions and challenges with integrating it into the tire [1]. These sensors may also change the dynamics of the tire due to their rigidity [34].

\subsubsection{Piezoelectric Sensors}

Sensors comprised of piezoelectric materials have also been used to measure deflection of tires $[2,17,18,42,54,72,73]$. Several systems have been patented to use piezoelectric sensors to measure tire deflections either to monitor tire pressure or tire-ground interaction $[10,47]$. Piezoelectric materials accumulate charge on their surface due to mechanical stress. By measuring the amount of charge accumulated, the stress can be inferred. Piezoelectric materials also have potential to be used to harvest energy from mechanical inputs, however they will not be used for that purpose in this study. Instead, the focus of this study is on using piezoelectric sensors to measure 'dynamic strain'.

The reaction of a piezoelectric material to mechanical stress $\sigma_{m}$ in the absence of an external magnetic field is

$$
D_{i}=d_{i j} \sigma_{j}
$$

where $D_{i}$ is the dielectric displacement and $d_{i m}$ is a matrix of the the piezoelectric strain coefficients $[40,62,69]$.

Typical values for these parameters are given in Table 2.1. The directions for the indices in the above equations are labeled in Figure 2.1 along with the dimensions of a film. Typically, piezoelectric films are used in the 1 direction for high frequency sensing and the 3 direction for low frequency sensing.

The total charge $q$ generated on the surface of the piezoelectric material is related to the dielectric displacement by

$$
q=\iint D_{i} \mathrm{~d} A_{i} .
$$

A piezoelectric film consisting of piezoelectric material with length $l_{p}$, width $w_{p}$, and thickness $t_{p}$ (as seen in Figure 2.1) with electrodes across the top and bottom surfaces can be treated 


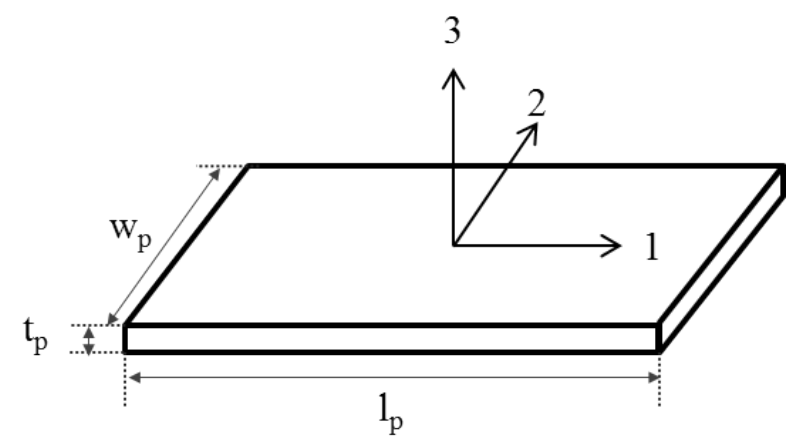

Figure 2.1: Dimensions and axes of a piezoelectric film sensor

Table 2.1: Physical and electrical characteristics of PVDF piezoelectric sensors.

\begin{tabular}{|c|c|c|c|}
\hline Symbol & Parameter & Value & Unit \\
\hline$Y$ & Young's modulus & typically $4-6$ & $\mathrm{GPa}$ \\
\hline$d_{31}$ & Piezoelectric strain coefficient & $23 \times 10^{-23}$ & $\frac{\mathrm{C}}{\mathrm{N}}$ \\
\hline$d_{32}$ & Piezoelectric strain coefficient & typically $2.5-3 \times 10^{-12}$ & $\frac{\mathrm{C}}{\mathrm{N}}$ \\
\hline$d_{33}$ & Piezoelectric strain coefficient & $-33 \times 10^{-23}$ & $\frac{\mathrm{C}}{\mathrm{N}}$ \\
\hline$R_{p}$ & Impedance at $10 \mathrm{kHz}$ & $12^{*}$ & $\mathrm{k} \Omega$ \\
\hline$p$ & Pyroelectric coefficient & $30 \times 10^{-6}$ & $\frac{\mathrm{C}}{\mathrm{m}^{2}} \mathrm{~K}$ \\
\hline$\frac{\epsilon}{\epsilon_{0}}$ & Dielectric constant & 10.7 & where $\epsilon_{0}=8.85 \frac{\mathrm{pF}}{\mathrm{m}}$ \\
\hline
\end{tabular}
Source: Measurement Specialties Inc [37], Sirohi and Chorpa [62]

* this value only applies to the DT1 sensing elements described in Section 3.2.

like a parallel plate capacitor with capacitance

$$
C_{p}=\frac{e_{33} l_{p} w_{p}}{t_{p}}
$$

where $e_{i j}$ is the dielectric permittivity or the piezoelectric material.

The voltage $V_{p}$ generated across a piezoelectric sensor with capacitance $C_{p}$ by the induced charge $q$ is

$$
V_{p}=\frac{q}{C_{p}}
$$

While a voltage can be detected across these sensors when there is a change in stress, this initial voltage signal $V_{0}$ will decay exponentially with time $t$

$$
V(t)=V_{0} e^{\frac{-t}{R_{p} C_{p}}}
$$

where $R_{p}$ is the electric resistance of the piezoelectric material. Because of this decay, static strain cannot be measured directly. Only changes in strain can be detected and will decay with time constant $\tau=R_{p} C_{p}$. It is for this reason that these sensors are called 'dynamic 
strain' sensors or 'strain rate' sensors. Signal conditioning circuits can be implemented with the sensors to increase the time constant, and therefore slow decay of the signal, as is demonstrated in Section 3.4.

From the voltage output of a piezoelectric film undergoing uniaxial strain, the amount of strain can be determined, as is clearly outlined in Sirohi and Chorpa [62]. When the film is undergoing multi-directional strain, the strain in each direction cannot be decoupled from the single combined signal.

The electromechanical properties of PVDF are dependent on the temperature of the sensor. PVDF is a pyroelectric material which means that charge will generate on its surface in response to changes in temperature [37,61]. The amount of charge generated for a piezoelectric film per unit change in temperature is given by the pyroelectric coefficient $p$ in Table 2.1. For a PVDF piezoelectric film with dimensions as in Figure 2.1, the amount of charge $q$ generated from a change in temperature $\Delta T$ is

$$
q=p \Delta T l_{p} w_{p}
$$

and the resulting voltage is

$$
V_{p}=\frac{p t_{p} \Delta T}{\epsilon} .
$$

This charge and voltage is subject to the same decay as was previously described.

Changes in temperature will have permanent and reversible and changes to the piezoelectric constants. Long term (days, weeks and years) exposure to elevated temperatures can cause a permanent decay of the piezoelectric strain constants. The piezoelectric strain constants, which govern the charge generation per unit of mechanical strain, will change based on temperature. This change is reversible. For a PVDF film, as temperature increases, the piezoelectric strain constants will increase. As temperature decreases, the piezoelectric strain constants will also decrease [37].

Piezoelectric film sensors are small, lightweight, and inexpensive. Because of their strong electrical sensitivity to mechanical strain, piezoelectric film sensors have potential to be used in tires to measure the forces acting on the tire. The charge generated by these sensors will decay with time. While the time constant of the decay can be extended with addition of an appropriate signal conditioning circuit, purely static strains cannot be measured with these sensors. The primary disadvantages to using piezoelectric film sensors are difficulties in decoupling strains in different directions and a strong sensitivity to temperature.

\subsection{Review of Previous Related Studies}

In this study, piezoelectric sensors were used on small tires on a wheeled mobile robot to evaluate the ground-terrain interaction. A review of similar previous studies is provided in this section, divided in two categories. In Section 2.3.1, studies that use non-tire-based sensors to characterize terrain are reviewed. In Section 2.3.2, studies that use piezoelectric sensors in car or small robot tires are reviewed. 


\subsubsection{Previous Studies Using a Wheeled Robot to Classify Terrain}

Several published studies demonstrate the potential of using a small wheeled robot to estimate soil parameters [14]. Nagatani et al. [44] used a $12 \mathrm{~kg}$ robot equipped with four rigid wheels with grousers. The wheels had normal force sensors embedded on the outside of the tires. Tests were conducted by traveling $1 \mathrm{~m}$ on Mars-like sand at different inclination angles. Through the sensor readings, the soil shear deformation modulus and the drawbar pull at different slip values were successfully estimated. The estimation required prior knowledge of the cohesion stress and the internal friction angle of the soil. While Iagnemma et al. [25] was able to estimate the cohesion stress and the internal friction angle from a force hub on the axle of a Mars rover-like wheel, this estimation required knowledge of the shear deformation modulus of the soil. From these studies, we see that some soil parameters can be identified if others are already known. In the field under unknown conditions, it is not expected that any soil parameters would be known.

In Ojeda [48], a Pioneer 2AT robot was instrumented with motor current sensors to determine terrain trafficability characteristics using Motor Current vs Rate of Turn (MCR) curves. This was expanded in Ojeda and Borenstein [49], where the robot was instrumented with inertial, motion (torque and moment), range, and other sensors. The measurements from the sensors were analyzed as part of a neural network to characterize terrain. Tests were conducted on gravel, sand, asphalt, grass, and dirt.

Ray et al. [54] used a custom-built, $13 \mathrm{~kg}$ robot instrumented with a variety of inertial, current, and other sensors to estimate wheel traction and torque using an extended KalmanBucy filter. The method was successfully demonstrated with a simulation and physical testing of the robot on a hard and a deformable terrain using 3 tread types with 2 foam inserts.

In Iagnemma et al. [25], there is a brief discussion of the challenges of measuring linear velocity in slow moving vehicle. Inertial measurement units (IMUs) are often used in larger vehicles to calculate velocity. The measurements from these systems can be degraded from noise at low velocities like those tested on a small robot. Instead, it is suggested to use visual odometry to measure velocity. This method is used in several other studies, however its applicability is limited to controlled laboratory applications.

\subsubsection{Previous Studies Using Piezoelectric Sensors in Tires}

In the study presented in this document, pneumatic tires are instrumented with sensors capable of measuring tire deflection. Several previous studies exist that use piezoelectric sensors in tires to characterize the surface.

In Moon et al. [42], a small semi-pneumatic tire was instrumented with a piezoelectric sensor clamped to the sidewall and another sensor glued to the tread. The tire was then attached to a test instrument that cyclically loaded the tire in the vertical direction. Results showed that the sensors were able to measure buckling of the sidewall and tread deflection. The tire used in this study was semi-pneumatic, meaning that it is air filled but that air is not compressed. The tire supports weight from the vehicle by the stiffness of its own 
material. It is expected that this type of tire will experience much greater deformation than a fully pneumatic tire of the same size.

The work from Moon et al. [42] was continued through implementing these sensors in a $10.9 \mathrm{~kg}$ robot. Two small piezoelectric sensors from Measurement Specialties were glued to the inside of the tire tread. The study authors predicted that the characteristic curve for each rotation would first have a negative charge dip, then a positive charge peak. The authors asserted that the negative dip occurs in the 'stick' region and is therefore caused by bending and stretching due to stick. As the sensor moves into the 'slip' region of the contact patch, the resulting positive peak is due only to bending. Therefore, by comparing the amplitudes of the negative and positive peaks, the amount of stretching of the sensors can be predicted. The stretching of the sensor is due to the tractive force which can indicate characteristics of the friction of the surface. The test sequence consisted of a series of spinning maneuvers to achieve a range of slip values. Tests were conducted on sand and concrete. Overall, the researchers had more success with characterizing the friction coefficient than with characterizing the slip. The friction coefficient estimation was required knowledge of the tire stiffness.

Data transmission from the inside to the outside of the tires was accomplished with the use of a wireless data transmission unit placed inside the semi-pneumatic tires. The robot was equipped with other sensors, including a 3 axis IMU, wheel encoders, and vision based location system. The vision system and wheel encoder were used to validate the slip predictions.

This paper is valuable because it demonstrates the use of piezoelectric sensors in a similar manner to what is proposed in this study. Several lessons can be learned from this study. The analysis was complicated by difficulties in characterizing the sensitivity of the signal conditioning circuit and by a low sampling rate. Additionally, the two sensors had slightly different results due to differences in attachment, placement and amplification sensitivity.

In Erdogan [16], Erdogan et al. [18], a test rig was developed to allow a car tire to move across a deformable surface as it would in driving conditions. The test tire was instrumented with a piezoelectric sensor in a cantilever beam configuration to directly measure lateral sidewall deflection. The signal were transmitted wirelessly to a DAQ board, then processed to measure the slip angle. The sidewall deflection measurements were verified using a camera mounted inside the tire and an LED on the moving sensor. The advantage to this sensor configuration was that it allowed for the deflections experienced by the to be decoupled, only measuring the sidewall deflection. 


\section{Chapter 3}

\section{Design of Test System}

The first objective of this study, described in Section 1.2, was to design a low cost small wheeled mobile robot for intelligent tire testing. In the following sections, I will describe the design process for the robot and the sensors, signal conditioning, and data acquisition system on the robot.

\subsection{Robot}

In order to test small tires, a robotic test system had to be developed. The following sections will describe the semi-custom kit from SuperDroid Robots (Fuquay-Varina, NC) and the reasoning behind the selection of this kit. I will describe the chassis of the robot, the motors, the motor controller, and the wheels and tires.

\subsubsection{Kit Selection}

Small wheeled robots can be described in three categories regarding their level of customization:

1. Off-the-shelf robots that are fully assembled and do not have options for customization

2. Semi-custom robots that come in kits with various options, similar to buying a new automobile from a dealership

3. Fully custom robots, fabricated for your specific needs.

One of the goals of this project is to design a robot that is be simple to reproduce to be used in the field. Fabricating a custom robot would not be consistent with this goal. A fully custom robot would be cost prohibitive and time prohibitive, due to the limited experience that this research group has with robotics. With this in mind, a list of the most basic requirements for the robotic test system was developed. These basic requirements were:

- four pneumatic tires, like a car 
- ability to acquire necessary data

- less than $\$ 2000$

- available off-the-shelf or semi-custom

Many other characteristics of candidate test systems were taken as considerations, but not requirements. A listing of all of the characteristics considered is provided in Table 3.1. Some of these items are related to the second basic requirement, the "ability to acquire necessary data".

The sources used to explore options of robotics companies were: search of the internet, consultation with student researchers in the Computational Multiphysics Systems (CMS) Laboratory at Virginia Tech, and a review of articles published about similar studies. The basic requirements listed above eliminated several broad categories of robots.

Hobby robots, while very affordable, were generally found to be too small to hold the necessary equipment due to size and load limits. The hobby robots considered before eliminating this category included Lynxmotion (Swanton, VT) Aluminum 4WD1 Rover Kit, DFRobot (Shanghai, China) 4WD Outdoor Mobile Platform, and the DFRobot 4WD Arduino Mobile Platform.

Many larger off-the-shelf robot kits were eliminated after they were found to be cost prohibitive. These companies along with the robot that best fit our needs are: Jaguar-4x4wheel Mobile Robotic Platform by Dr. Robot (Markham, ON) (over \$3000), the Pioneer 3-AT by Adept Mobile Robots (Amherst, NH), MMP-30 by the Machine Lab (Fort Collins, $\mathrm{CO}$ ), and everything at Applied Research Associates (Albuquerque, NM). The Pioneer 2-AT and 3-AT is popular and is used in other scientific studies [12, 48, 49].

Several other robotics companies investigated do not currently make robots with four wheels. These companies include Mekatronix (Gainesville, FL), Arrick Robotics (Tyler, TX), Parallax (Rocklin, CA), K-Team Corporation (Vallorbe, Switzerland), and AcroName (Boulder, CO). VEX (Greenville, TX) and Nexus (Guangdong, China) do not offer robots with pneumatic tires.

Two companies were identified that create semi-custom robot kits that fit our basic requirements: the All-Terrain Robot kits from SuperDroid Robots (Fuquay-Varina, NC) and the Surface Mobility Platform by Gears Educational Systems (Pittsburg, KS). Both of these systems have been previously used in the CMS laboratory at Virginia Tech. These two systems are compared in Table 3.2, along with typical hobby cars for reference. GEARS has other four wheeled kits that were eliminated for other reasons.

Because of the variety of pneumatic tire types available and ability to add slip rings, as described in Section 3.3.1, the Heavy Duty All Terrain Mobile Robot Platform from SuperDroid Robots (Fuquay-Varina, NC) was selected. The small wheeled robot used was developed from a semi-customized kit. This brand appears to have been used in other related studies [54, 72]. The four wheel construction allows the dynamics of the vehicle to be similar to that of an automobile. The cost was low and the company, located in nearby North Carolina, was found to be responsive to our needs to modify certain components. The 
Table 3.1: Criteria considered during selection of a robot or robot kit.

\begin{tabular}{|c|c|}
\hline Characteristic & Comment \\
\hline \multicolumn{2}{|l|}{ Structural } \\
\hline $\begin{array}{l}\text { Off-road } \\
\text { capability }\end{array}$ & Yes \\
\hline Durability & Durable \\
\hline Size & $\begin{array}{l}\text { Able to be lifted by a single adult without additional equipment, } \\
\text { space to fit a laptop }\end{array}$ \\
\hline $\begin{array}{l}\text { Axle size and } \\
\text { layout }\end{array}$ & Able to add slip rings \\
\hline \multicolumn{2}{|l|}{ Mechanical } \\
\hline Number of tires* & Four pneumatic tires \\
\hline Rated load & $\begin{array}{l}\text { Able to hold a laptop, DAQ, hard drive and allow future } \\
\text { expansion of test system, including possible ballast weight }\end{array}$ \\
\hline Tire options & Multiple tire options available, able to easily change tires \\
\hline Braking & Available electronic braking \\
\hline Steering method & Turning wheels preferred to differential or skid steered \\
\hline Maximum torque & Able to drive with high slip on deformable off-road surfaces \\
\hline Maximum speed & Able to drive with high slip on deformable off-road surfaces \\
\hline \multicolumn{2}{|l|}{ Electronics } \\
\hline Speed control & Programmatically and by handheld radio \\
\hline Sensors & Prefer built in encoders, inertial sensors, motor current sensors \\
\hline $\begin{array}{l}\text { Signal } \\
\text { conditioning }\end{array}$ & Preferred to be built in \\
\hline DAQ & Preferred to be built in \\
\hline \multicolumn{2}{|l|}{ Other } \\
\hline Cost* & Less than $\$ 2000$ \\
\hline $\begin{array}{l}\text { Level of } \\
\text { customization* }\end{array}$ & Off-the-shelf or semi-custom \\
\hline $\begin{array}{l}\text { Ability to acquire } \\
\text { necessary data* }\end{array}$ & Yes \\
\hline Customer support & Helpful and accessible \\
\hline Delivery time & Less than two weeks \\
\hline
\end{tabular}


Table 3.2: Comparison of capabilities of typical hobby cars, the SuperDroid Heavy Duty ATR and the GEARS SMP.

\begin{tabular}{|c|c|c|c|}
\hline Characteristic & $\begin{array}{l}\text { Typical } \\
\text { Hobby } \\
\text { Cars }\end{array}$ & $\begin{array}{l}\text { SuperDroid Heavy } \\
\text { Duty ATR }\end{array}$ & GEARS SMP \\
\hline \multicolumn{4}{|l|}{ Structural } \\
\hline Off-road capable & varies & yes & yes \\
\hline Durable & no & yes & yes \\
\hline Size and weight (can be lifted) & yes & yes $(13.2 \mathrm{~kg})$ & yes $(8.2 \mathrm{~kg})$ \\
\hline Size (can hold computer) & no & yes, wide platform & $\begin{array}{l}\text { maybe, small } \\
\text { platform }\end{array}$ \\
\hline Ability to add slip rings & limited & $\begin{array}{l}\text { requires slight } \\
\text { modification }\end{array}$ & $\begin{array}{l}\text { requires extensive } \\
\text { modification }\end{array}$ \\
\hline \multicolumn{4}{|l|}{ Mechanical } \\
\hline Four wheels* & yes & yes & yes \\
\hline Pneumatic tires & most no & yes & $\begin{array}{l}\text { only foam insert } \\
\text { tires }\end{array}$ \\
\hline Rated load (lb) & $\begin{array}{c}\text { about } \\
10\end{array}$ & $25-40$ & 18 \\
\hline Tire options & varies & $\begin{array}{l}6 " \text { or } 10 " \text { diameter } \\
\text { pneumatic, easy to } \\
\text { change }\end{array}$ & $\begin{array}{l}4.5 ", 6 ", \text { or } 8 " \\
\text { foam insert, } \\
\text { easy to change }\end{array}$ \\
\hline Braking & limited & no & no \\
\hline Steering method & varies & differential & differential \\
\hline \multicolumn{4}{|l|}{ Electronics } \\
\hline Speed control & $\begin{array}{l}\text { radio, } \\
\text { lim- } \\
\text { ited }\end{array}$ & $\begin{array}{l}\text { radio or } \\
\quad \text { programmatically }\end{array}$ & not specified \\
\hline Sensors built in & no & $\begin{array}{l}\text { encoders optional, } \\
\text { motor controller } \\
\text { measures current }\end{array}$ & not standard \\
\hline Ability to add sensors & yes & yes & yes \\
\hline Signal cond or DAQ built in & no & no & no \\
\hline Ability to add signal cond & yes & yes & yes \\
\hline \multicolumn{4}{|l|}{ Other } \\
\hline Cost* & $\$ 50-500$ & about $\$ 1500$ & about $\$ 1500$ \\
\hline Customizable* & varies & semi-custom & semi-custom \\
\hline Customer support & $\begin{array}{l}\text { yes, } \\
\text { varies }\end{array}$ & yes, responsive & unknown \\
\hline Delivery time & varies & $\begin{array}{l}\text { ships in } 3-5 \text { days from } \\
\text { about } 200 \mathrm{mi}\end{array}$ & not specified \\
\hline
\end{tabular}

Source: Gears Educational Systems [20], SuperDroid Robots Inc. [63]. 
robot was ordered as a kit that I later assembled. The parts comprising the kit and other components added to the system are described in the following subsections.

\subsubsection{Chassis}

The standard chassis on the Heavy Duty ATR from SuperDroid was used. It is constructed of welded high strength steel that forms a $16 " \times 12$ " $\times 3$ " box that can be seen in Figure 3.1a. The thickness of the steel is 0.25 ". The chassis has four motor mounts at offset positions. After receiving the chassis from SuperDroid, a hole was drilled through the top surface to route the wires from the motor mounts on the underside to the motor controller on the top. On the top side of the chassis, a metal box was added to house the motor controller, DAQ, and radio receiver.

\subsubsection{Motors and Encoders}

SuperDroid offered several motor options for the Heavy Duty ATR kit. Our requirement was that the motors have enough strength to create a high slip condition and to drive the robot on deformable surfaces.

Four 24 V D.C. carbon-brush planetary gear motors from Shayang Ye Industrial Company (Taoyuan, Taiwan) were supplied by SuperDroid. The specifications provided on the product datasheet [60] are shown in Table 3.3. Due to space constraints, the motors were coupled to the wheels by a sprocket-chain mechanism, shown in Figures 3.1b and 3.2.

Table 3.3: Specifications for the IG42 $24 \mathrm{~V}$ motor.

\begin{tabular}{lrl}
\hline Parameter & Value & Unit \\
\hline Supply voltage & 24 & $\mathrm{~V}$ \\
Max torque & 570 & g-cm \\
Max speed & 5900 & $\mathrm{rpm}$ \\
Current & $\leq 2100 \mathrm{~mA}$ \\
No load speed & $7000 \mathrm{rpm}$ \\
No load current & $\leq 500 \mathrm{~mA}$ \\
Power output & 34.7 & $\mathrm{~W}$ \\
\hline
\end{tabular}

Source: Shayang Ye Industrial Co. Ltd. [60]

Each motor has an attached two channel Hall effect encoder that give 5 pulses per revolution (PPR) of the encoder. A photograph of a motor and the attached encoder is show in Figure 3.3. 


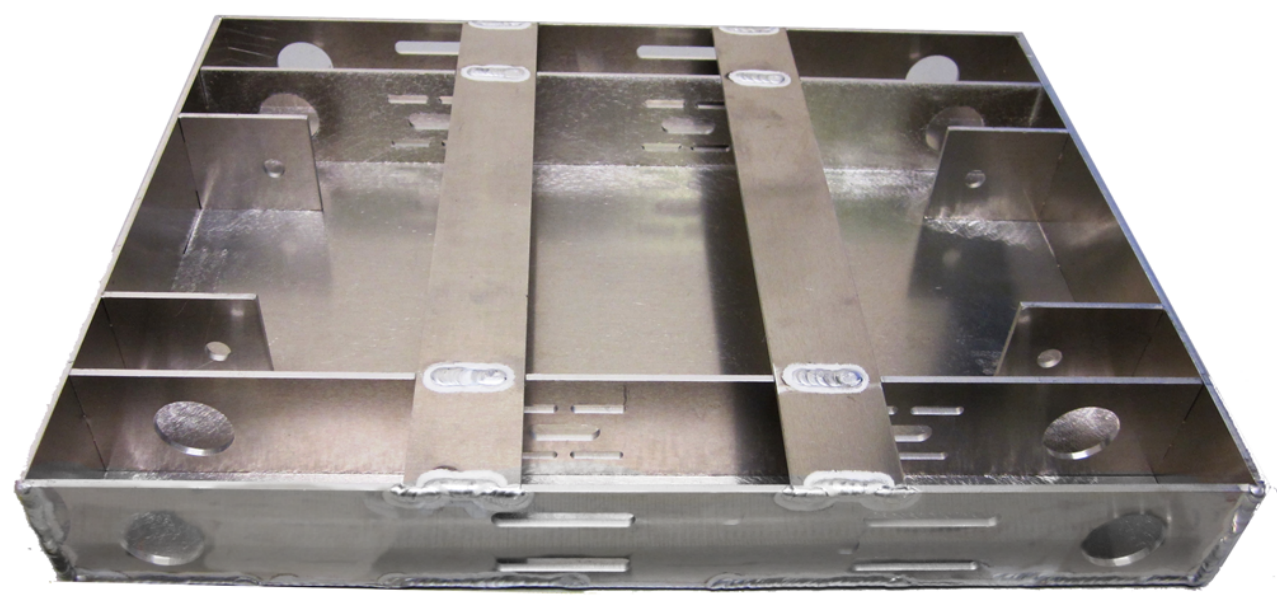

(a)

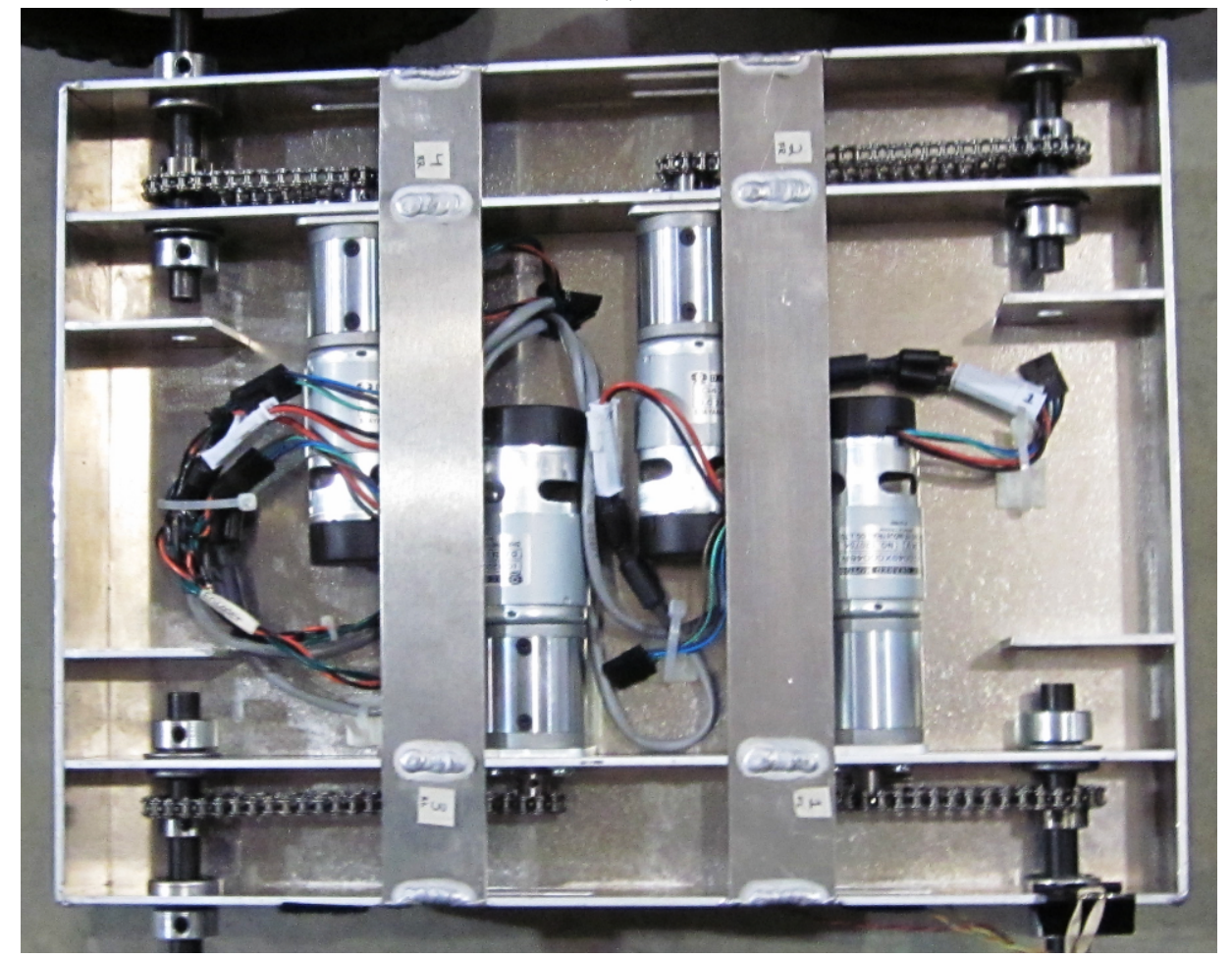

(b)

Figure 3.1: Photograph of underside of Heavy Duty ATR chassis by SuperDroid. (a) Unassembled, as it was sent in the kit and (b) With motors and chains mounted 


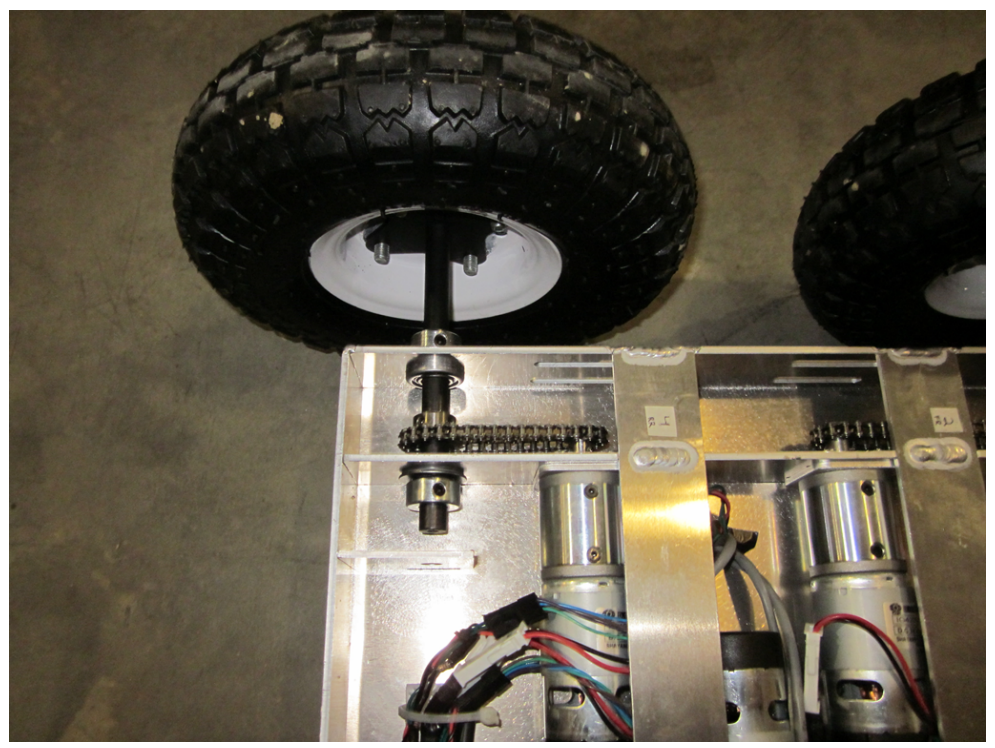

Figure 3.2: Photograph of chain-sprocket mechanism connecting motors to wheel hubs.

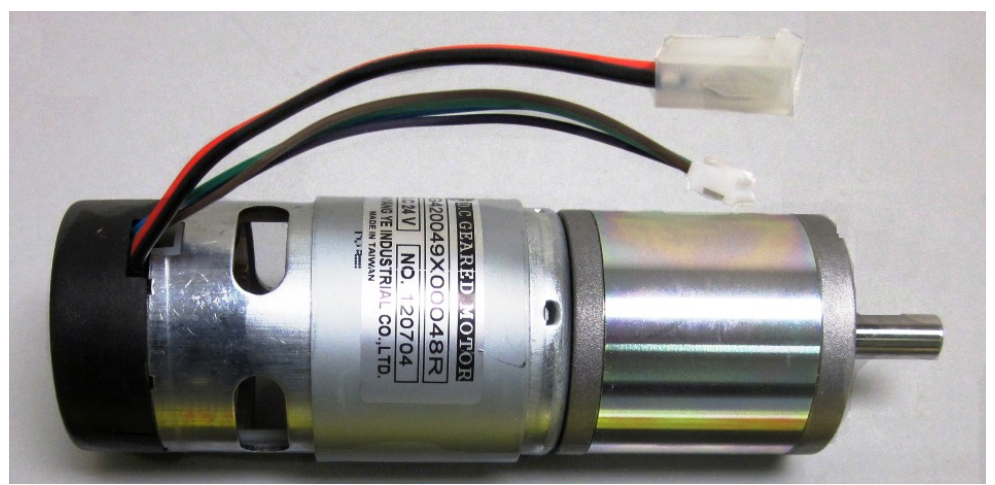

Figure 3.3: Photograph of a 24 V, 120 RPM motor with attached two channel Hall effect encoder. 


\subsubsection{Motor Controller}

The purpose of the motor controller in the robotic system is to interpret command inputs from a computer, pre-loaded script, or handheld radio controller and use those inputs to distribute power from the battery to the motors. SuperDroid offers several brands and models of motor controllers that vary based on the following features:

- allowed size of supply voltage

- number of output channels (1 or 2$)$

- presence of a $5 \mathrm{~V}$ analog output to power encoders or other devices

- presence of pulse width modulated radio input channel

- available USB connection to computer

- ability to interpret encoders to operate in a closed loop mode

The SDC2130 motor controller from Roboteq (Scottsdale, AZ), shown in Figure 3.4, was selected from SuperDroid. The Roboteq SDC2130 is a two channel motor controller. One channel controls the two right side wheels together and the other channel controls the two left side wheels together. The available inputs for the controller are a USB connection and a serial connection via a DB15 female port [55].

The motor controller allowed the robot to be controlled in a closed loop speed or open loop power mode. Power mode was selected due to certain limitations of the speed mode with the wiring configuration of the robot. The motor controller was wired to power four wheels, wired as pairs in parallel. In this configuration, one power level is applied to both right side tires and another power level is applied to both left side tires. The power distribution applied to the front and the rear tires on a given side cannot be differentiated. The motor controller only has two inputs available for encoders. This means that while controlling four tires in closed loop speed mode, the controller would only be getting feedback from one tire per side. Due to this limitation, I chose to operate in open loop power mode.

The Roboteq SDC2130 was programmed to take inputs from a radio receiver connected to the DB15 port and from a computer on-board the robot via a USB cable. The controller was set to receive one command for speed and another command for direction. The motor controller has the ability to hold compiled MicroBasic code which it will execute at a command from the serial or USB. This is how the robot was given a controlled signal.

The two motor encoders were connected to the serial port. This was necessary because the DAQ, described in Section 3.3.2 had only one event counter input, which is the type of input needed for an encoder. While this controller has the capability to operate in a closed loop mode with an encoder feedback, this feature was not used. The number of pulses of the motor encoders per revolution of the tire was

$$
\mathrm{PPR}=4 \mathrm{PPR}_{\text {encoder }} \mathrm{GPR}_{\text {motor }} \mathrm{GPR}_{\text {chains }}
$$




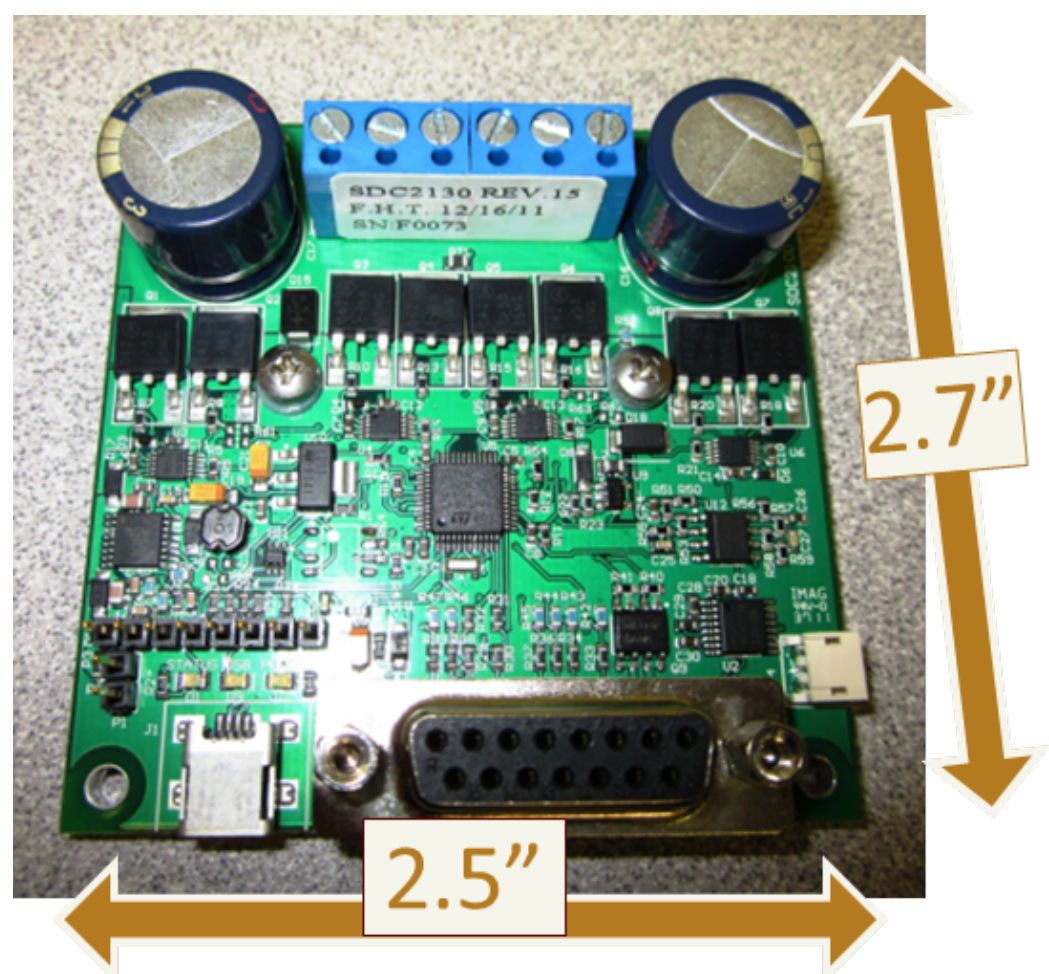

Figure 3.4: Photograph of Roboteq SDC2130 motor controller.

where $\mathrm{PPR}_{\text {encoder }}$ is the pulses of the encoder per revolution (5, provided in the datasheet [59]), GPR $\mathrm{Gotor}_{\text {is }}$ the gear reduction ratio of the motor (49, provided in the datasheet [60]), and $\mathrm{GPR}_{\text {chains }}$ is the reduction ratio of the chains (counted, 14/10). The result is $343 \mathrm{PPR}$.

The user manual for the motor controller contains a recommendation that the encoder consumes less than $50 \mathrm{~mA}$ of current. The maximum pulse frequency that the controller can handle is $100 \mathrm{MHz}$ which we are well within. The connectors purchased and used for the encoders are listed in Appendix A.

\subsubsection{Wheels and Tires}

One of the advantages of using the SuperDroid ATR was that there were multiple tire types available. The SuperDroid Heavy Duty ATR has four wheels. Each wheel consists of a 0.5 in wheel shaft that is driven via a chain mechanism by a motor. There were two pneumatic tire sizes available from SuperDroid: 6" diameter and 10" diameter. A comparison of the tires is provided in Table 3.4. The two types of tires use different types of shafts. The only customization made to the order was that the shafts be fabricated 1" longer than usual to allow space for the slip rings. Figure 3.5 shows the two types of treads and Figure 3.6 gives a side view of the tires. The tread patterns are very different between the two tires. The 6" tires have a small parallel zig-zag pattern that suggests it may be more designed for indoor applications. The 10" tires appear to be more rugged, with a deep 'knobby' tread. This 
Table 3.4: Description of the two pneumatic tires used in this study. The tires were acquired in the kit from SuperDroid.

\begin{tabular}{lll}
\hline Parameter & Small tire & Large tire \\
\hline Diameter & 6 in & $10 \mathrm{in}$ \\
Width & 2 in & 4 in \\
Tire type & pneumatic, tube & pneumatic, tube \\
Load capacity & $300 \mathrm{lb}$ & $300 \mathrm{lb}$ \\
Rated pressure & $30 \mathrm{psi}$ & $30 \mathrm{psi}$ \\
\hline
\end{tabular}

Source: Northern Tool and Equipment [46]

tread may be designed for outdoor applications.

The tires used in this study were acquired from SuperDroid but are also available from the retailer Northern Tool + Equipment (Burnsville, MN). They are described on the Northern Tool website as "industrial tires" and are used in the field for everything from hand carts to personal walkers. Both tire types are pneumatic with an inflatable inner tube. The writing on one of the tires indicates that it was made in China and that it is a "nylon tube type", although it is unclear if this means that the tubes are nylon or the tire is nylon. Both tires appear to be constructed from a mold. The tires are held to the hubs by two metal plates that sandwich them from both sides. A hole was drilled in one of the plates for the sensor leads. 


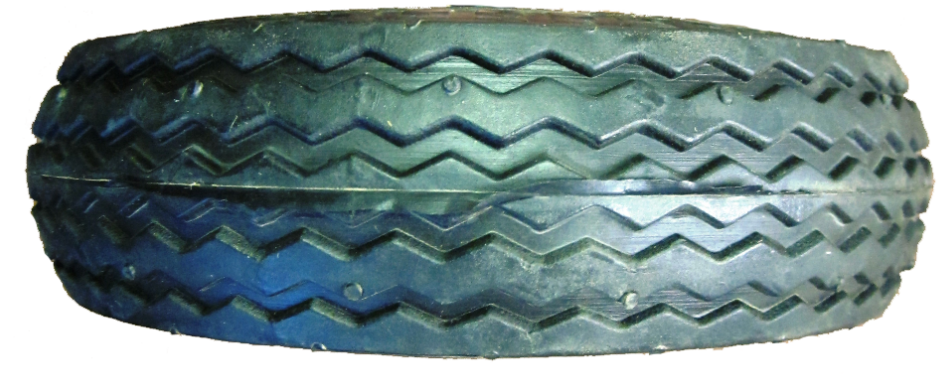

(a)

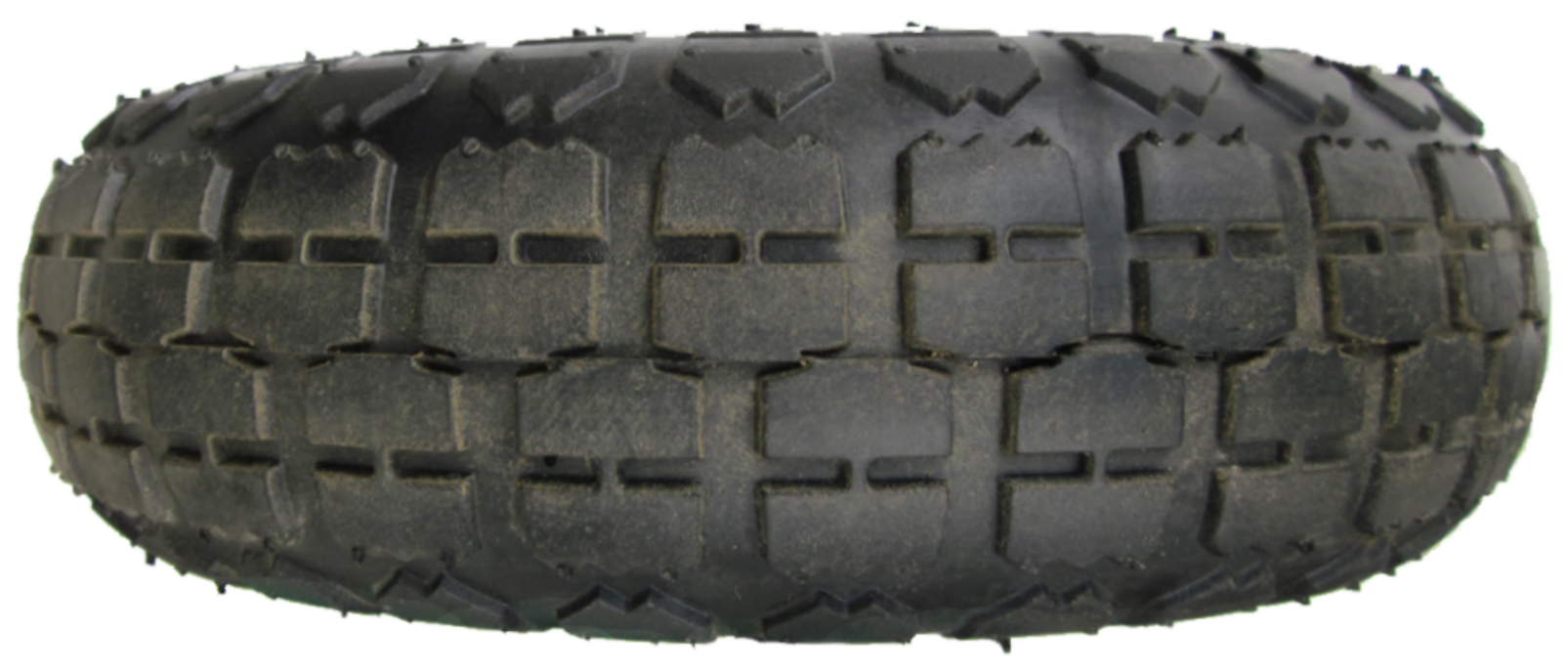

(b)

Figure 3.5: Photographs of the two tire treads.

a) 6", b) $10 "$ 


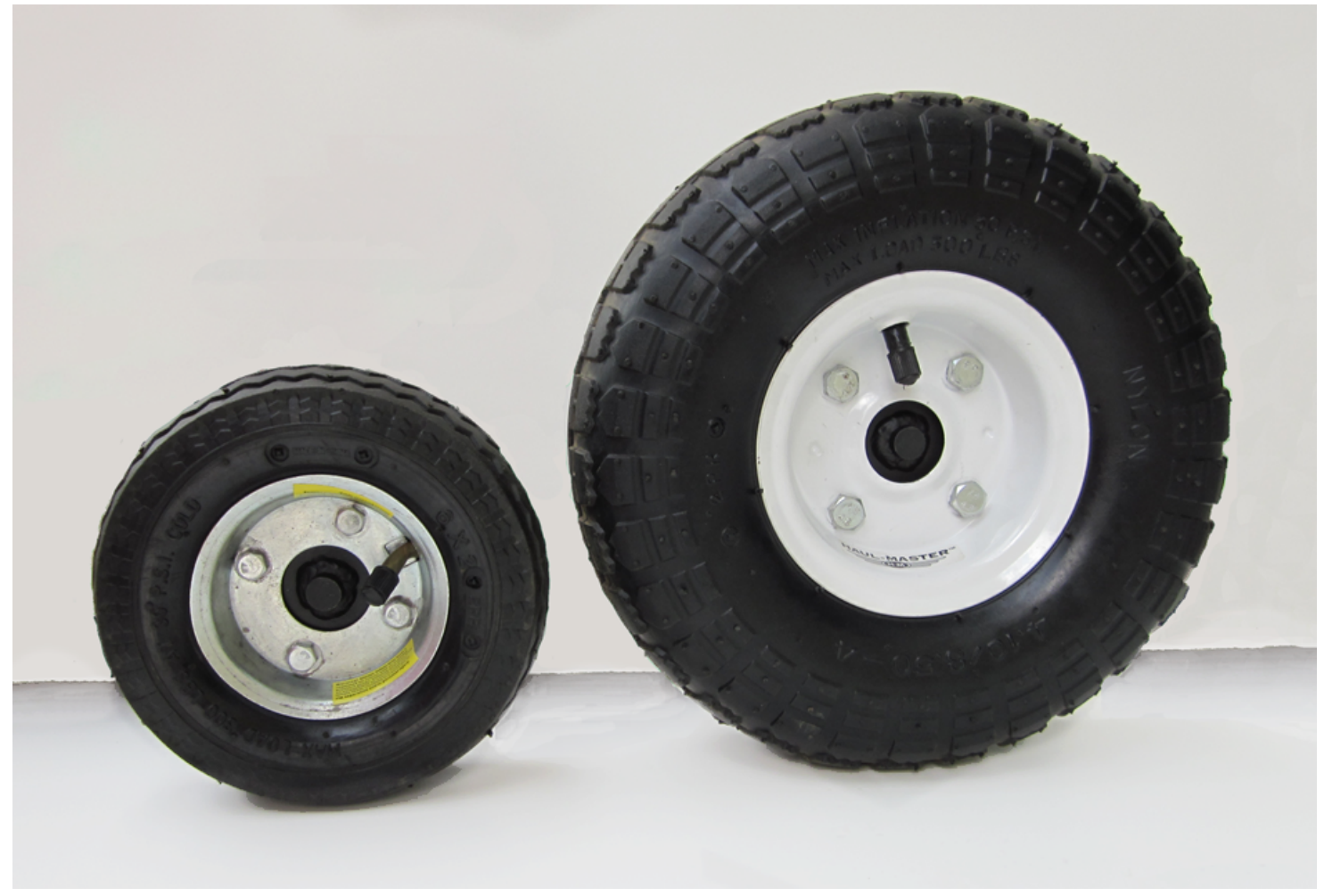

Figure 3.6: Photographs of the two tire sides with the 6" tire on the left and the 10" tire on the right. 


\subsection{Piezoelectric Sensors}

The second objective of this study is to instrument the tires of the robot with sensors to characterize the tire-ground interaction. Piezoelectric sensors from Measurement Specialties (Hampton, VA) were selected due to their low cost, commercial availability, and ability to generate an electrical signal in response to mechanical strain. Sensors from this company have been used in other studies [73]. A discussion of piezoelectric sensors was included in Section 2.2.4. Piezoelectric sensors use the concept of piezoelectricity, which is charge accumulating on the surface of a material as a result of mechanical stress [40,62]. There are several different variations in sizes and characteristics of the piezoelectric films that may be more sensitive to different measurement parameters. The film sensors from Measurement Specialties have a layer of PVDF piezoelectric material sandwiched between two silver electrodes to collect any charge generated. The particular products chosen for further examination are described in Table 3.5.

Table 3.5: Piezoelectric sensors considered from Measurement Specialties.

\begin{tabular}{lccccccc}
\hline product number & $\begin{array}{c}t_{s} \\
(\mu \mathrm{m})\end{array}$ & $\begin{array}{c}w_{f} \\
(\mathrm{~mm})\end{array}$ & $\begin{array}{c}w_{p} \\
(\mathrm{~mm})\end{array}$ & $\begin{array}{c}l_{f} \\
(\mathrm{~mm})\end{array}$ & $\begin{array}{c}l_{p} \\
(\mathrm{~mm})\end{array}$ & $\begin{array}{c}\mathrm{C} \\
(\mathrm{nF})\end{array}$ & laminated \\
\hline DT1-028K/L w/rivets & 40 & 16 & 12 & 41 & 30 & 1.38 & no \\
DT1-052K/L w/rivets & $\mathbf{6 4}$ & 16 & 12 & 41 & 30 & $\mathbf{0 . 7 4}$ & no \\
DT2-028K/L w/rivets & 40 & 16 & 12 & $\mathbf{7 3}$ & $\mathbf{6 2}$ & $\mathbf{2 . 7 8}$ & no \\
LDT1-028K/L w/rivets & $\mathbf{2 0 5}$ & 16 & 12 & 41 & 30 & 1.38 & yes \\
\hline
\end{tabular}

Source: Measurement Specialties Inc [38]

Note: Items in bold are those characteristics that differ from the'DT1-028K/L w/rivets' model. See Figure 2.1 for a diagram showing the length $l_{p}$ and width $w_{p}$ of the active sensor area. The dimensions of the thin coating surrounding the electrode are given with 'f' subscripts. Thickness $t_{s}$ is of the entire sensor outside the coating and/or lamination.

\subsubsection{Strains Experienced by Piezoelectric Sensors on a Tire}

As was explained in Section 2.2.4, one of the challenges to using piezoelectric film sensors is the difficulty of decoupling a multi-directional strain into its uni-directional components. A review of the strains that may be experienced by sensors in different configurations on a tire is presented here.

In this study, the piezoelectric films were placed outside of the inner tube of the tire, inside of the tire material itself. The compressed air in the inner tube would effectively sandwich the sensor between the tube and the tire. This would cause the sensor to experience normal pressure in the 3 direction due to the compressive force. The sensor will generate a signal while the tire is being inflated, but this signal will decay due to the time constant of the sensor and signal conditioner. 
First we will consider the strain experienced by a sensor placed circumferentially or laterally along the tread of the tire. The forces at the tire-ground interface may transfer through the tire material and result in strains on the sensor. As the sensors on the tire tread rotate through the contact patch, they will experience an increase in normal pressure in the 3 direction. This normal pressure is converted into longitudinal extension of the sensor in the 1 direction which is more electrically sensitive to strain (see the piezoelectric strain constants in Table 2.1). This longitudinal extension will cause charge to accumulate on the surface of the electrodes. As the sensors rotate through the contact patch, the longitudinal tractive force $F_{x}$ may transfer through the tire material resulting in a strain on the sensor in the 1 or 2 directions (for circumferential or lateral sensor orientations, respectively). During turning, a lateral force $F_{y}$ is generated at the tire-ground interface. The piezoelectric film sensor will experience strain in the 2 or 1 directions (for circumferential or lateral sensor orientations, respectively). The amount of strain in the longitudinal and lateral directions experienced for a given $F_{x}$ and $F_{y}$ will depend on the properties of the tire and the adhesive between the sensor and the tire. Piezoelectric films are most sensitive electrically in the 1 direction. Therefore, for applications where the longitudinal strain is of interest, the sensor should be placed in the circumferential direction. For applications where the lateral strain is of interest (such as during turning maneuvers), the sensor should be placed in the lateral direction.

Sensors could also be placed along the sidewall of the tires. As the tire rotates through the contact patch, the sidewall will deflect, causing strains within the sensor and therefore charge to accumulate on the sensor. While there will be some sidewall deflection caused by tractive or turning forces, it is expected that these would be small compared to the sidewall deflection caused by forces normal to the tread.

As the tire tread flattens both longitudinally and laterally in the contact patch, a sensor placed circumferentially or laterally in the tread will experience bending as it flattens. By adding a sheet of lamination to one side of the sensor, the neutral axis is moved out of the sensor and into the lamination. This causes the strains in the piezoelectric film to be greater during bending than without the lamination. It is therefore expected that a laminated sensor placed on the tire tread will be sensitive to deflection of the tread. As was stated before, the amount that a lateral or longitudinal shear force transfers from the tire-ground interface to the sensor depends on the properties of the tire and the adhesive. It is possible that the lamination may also affect the ability of these shear forces to transfer to the sensor.

\subsubsection{Planned Placement of Piezoelectric Sensors}

The differences in sensitivities of the laminated and unlaminated sensors may point toward a way to isolate the forces acting on the tire tread. If the amount of tread deflection is related to the normal pressure, then a laminated sensor placed along the tread will have signal related to the normal pressure. An unlaminated sensor placed along the tread will have a signal strength related to both the normal pressure and the shear forces caused by $F_{x}$ and $F_{y}$.

Based on this idea, three sensor configurations were selected for further testing. The 
'DT1-028K/L w/rivets' piezoelectric sensor was seen as the baseline for all of the sensor options that were available. It is the smallest sensor available in this series of sensors, based both on surface area and thickness. This sensor was selected for a comparison between an unlaminated and laminated sensor. The laminated version of the 'DT1-028K/L w/rivets' is the, 'LDT1-028K/L w/rivets'. Because there were no plans to test any turning maneuvers, it was decided to place the sensors so that their 1 direction aligned with the longitudinal direction of the tire, rather than the lateral direction. Another 'DT1-028K/L w/rivets' was placed on the inner sidewall of the tire. The intent of this placement is to measure sidewall bending. A diagram with the types and locations of the sensors is shown in Figure 3.7.

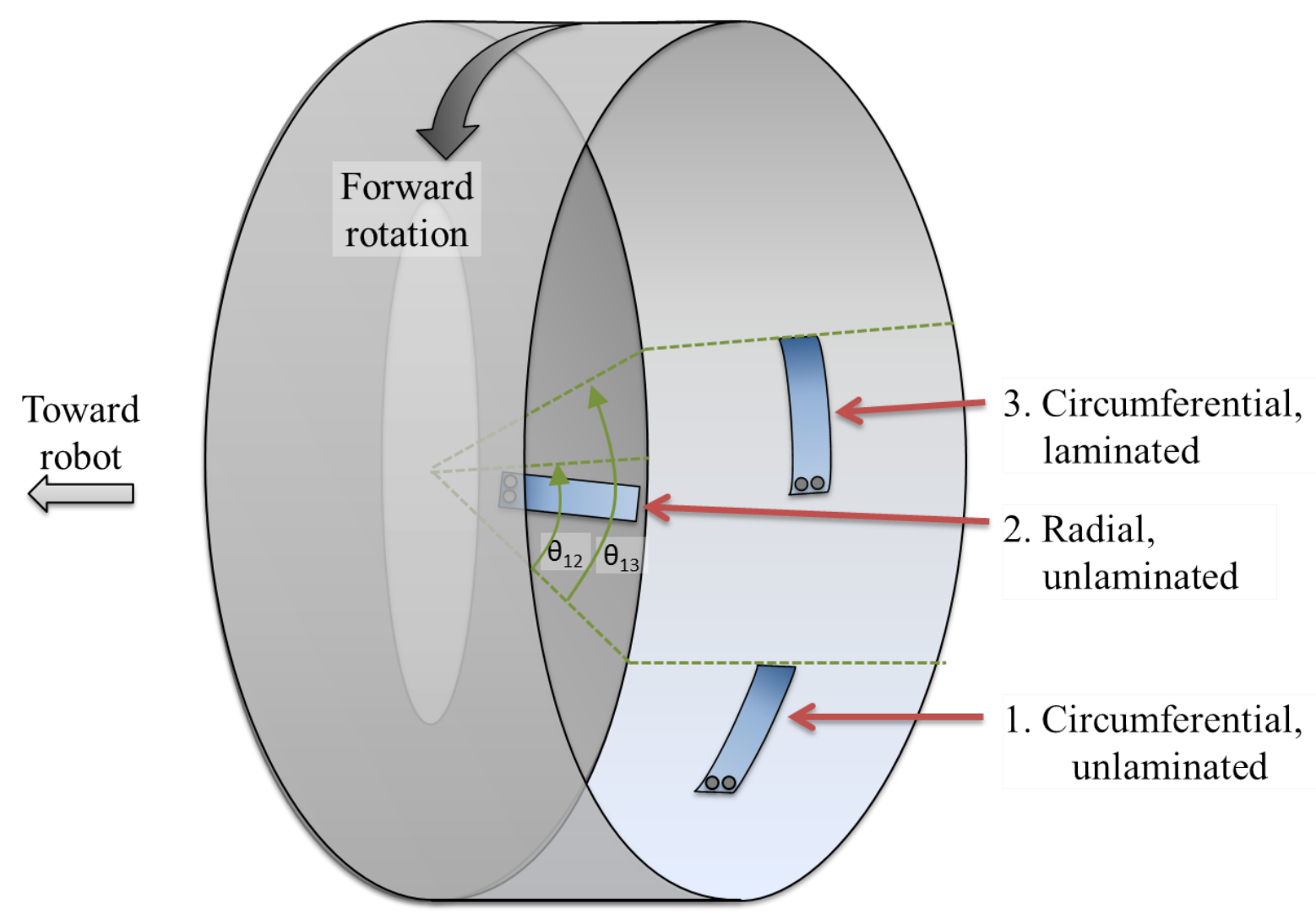

Figure 3.7: Diagram of mounting locations and types of piezoelectric sensors used in testing.

Note: Small circles on each sensor indicate locations of rivets for wire connections.

\subsubsection{Investigation Into Possible Adhesives For Sensors Attach- ment}

The method of adhering the piezoelectric film to the inside of the tire is important because the transfer of forces to sensor goes through the adhesive. In previous work with similar sensors, a clamping mechanism is used to attach the sensor the the substrate. This is not suitable for the present application. In Yi and Liang [73], glue was used to adhere similar, 
smaller sensors to the inside of a tire. One concern with using glue is that it is difficult to characterize and to adhere with a uniform thickness. Instead, I decided to use double sided tape, which would provide a uniform thickness and known physical properties. Measurement Specialties provides a list to customers of possible adhesives. This list was created in 2006 and was found to be outdated. I conducted an investigation into other types of double sided tape currently available.

Some double sided tapes consist of a carrier film that has adhesive on either side with a removable liner around that. Other double sided tapes are simply a single layer of adhesive, stored between two liners that are removed when the tape is used. The type with a single layer of adhesive, known as transfer tape, will always have the same adhesive strength on both sides of the tape. For tapes with a carrier film, it is possible to have different adhesive strength on each side. These are called differential tapes. The ability to have varying adhesive strengths is something not possible with glue.

The adhesive strength that will be required to bond an adhesive to a certain substrate depends on the surface energy of the substrate. Low surface energy (LSE) substrates are difficult to bond whereas high surface energy (HSE) substrates are easy to bond. Tapes are marketed as being able to bond to "low", "medium", or "high" surface energy substrates. The Nylon construction of our tires is probably a low surface energy material and the piezoelectric sensors are probably low to medium surface energy.

When selecting a tape, it is important to identify whether the intent is for the adhesive to be permanent or removable. For this study, the sensors should be removable from the inside of the tires. This will allow for testing of different configurations of the sensors and to be able to use the same tires for future studies that may not require the same sensors.

3M (St. Paul, Minnesota) has a wide selection of double sided tapes, so I chose to investigate options from this company more thoroughly. 3M Repositional Tape 665 and 666 were identified as candidates. These tapes have low tack on one side and high tack on the other and has medium bonding strength to both HSE and LSE [4]. I could only find these tapes in large industrial size quantities. Instead, a similar tape, 3M 667 was chosen. Tape 667 is readily available at commercial outlets as an office supply product. This tape is 2.4 mils thick [3].

\subsection{Data Transmission and Acquisition}

In order to make use of the signals from the piezoelectric sensors, the signal had to be transmitted from the tire to the robot body to be read by a DAQ. The signal from the DAQ is then converted from analog to digital and sent to an on-board computer by a USB cable. Two figures are provided to improve understanding of the data connections that are explained throughout this section. Figure 3.8 contains a block diagram of the components of the system. Figure 3.9 contains a diagram of the sensor layout within the tire and its connections to the on-board electronics. 


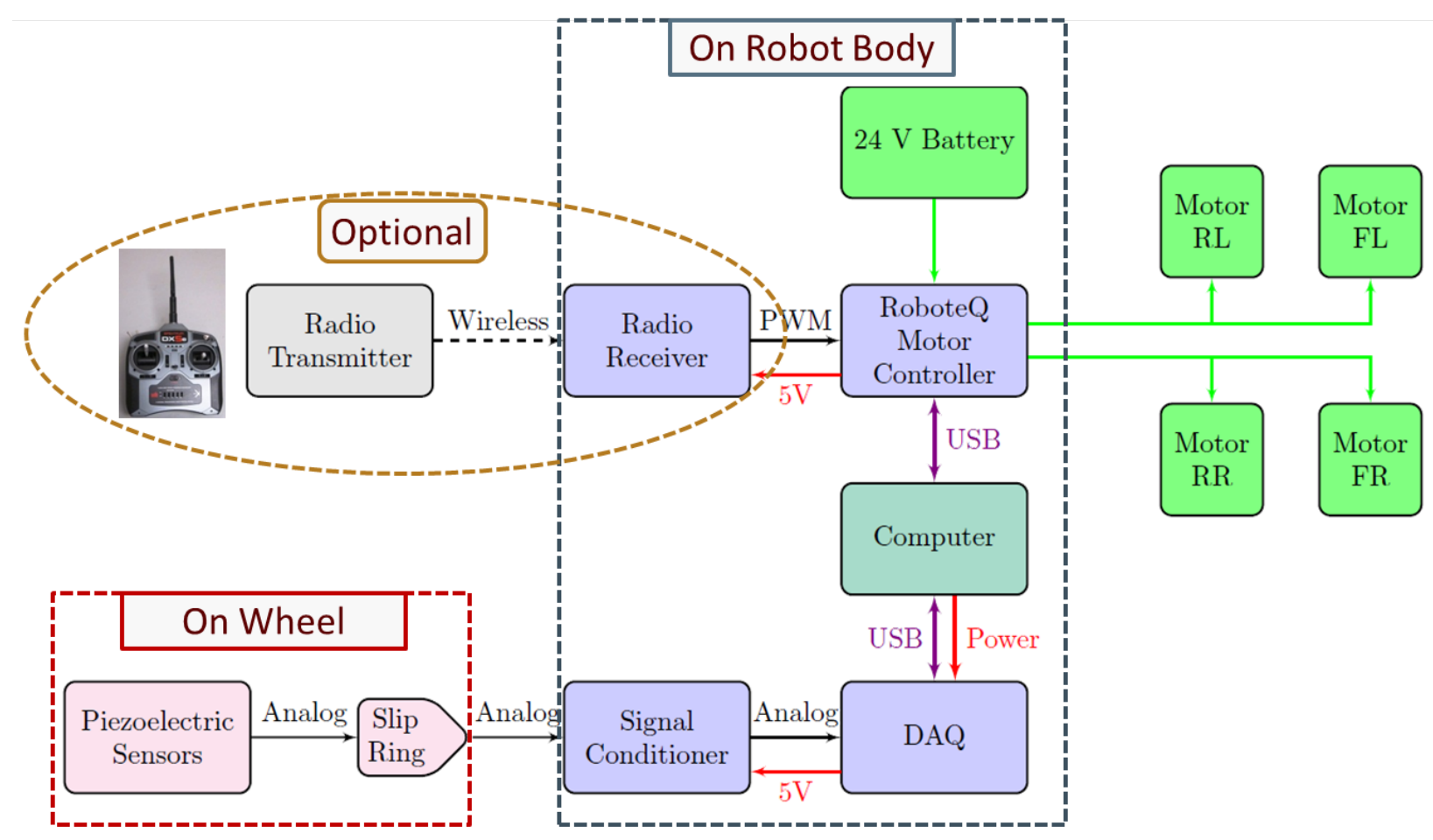

Figure 3.8: Block diagram of the test system components and their relative connections.

\subsubsection{Slip Rings}

An inherent challenge that arises when using sensors inside of tires is the need to transmit data from a rotating tire or wheel to non-rotating components. In some studies, this is done using wireless data transmission [18, 22, 23, 72]. Wireless transmitters require a power source, meaning that there must also be a battery on the wheel or tire. Instead, the use of a slip ring to transmit data through wired connections without conversion to wireless was investigated. A slip ring is a electromechanical unit that allows for wires to rotate and maintain an electrical connection without getting twisted or tangled. Slip rings with through bores can be mounted on the axle of a rotating part. The wires coming out of one end of the slip ring rotate with the axle while the wires on the other end remain fixed. The wires on either side maintain an electrical connection with one another.

Four SRA-73683-6 slip rings were provided by Moog (Blacksburg, VA). The SRA-73683-6 features a 0.5 in through bore enabling it to be securely mounted on both of the standard diameter shafts from SuperDroid. Recall from Section 3.1.5 that the wheel shafts were ordered to be longer in order to accommodate the slip rings. A picture of the slip ring is provided in Figure 3.10 and the specifications for the Moog SRA-73683-6 are given in Table 3.6.

The wires were twisted into three sets of pairs on either side. The connectors selected for connections from slip ring to sensor and slip ring to signal conditioner are indicated in Appendix A. 


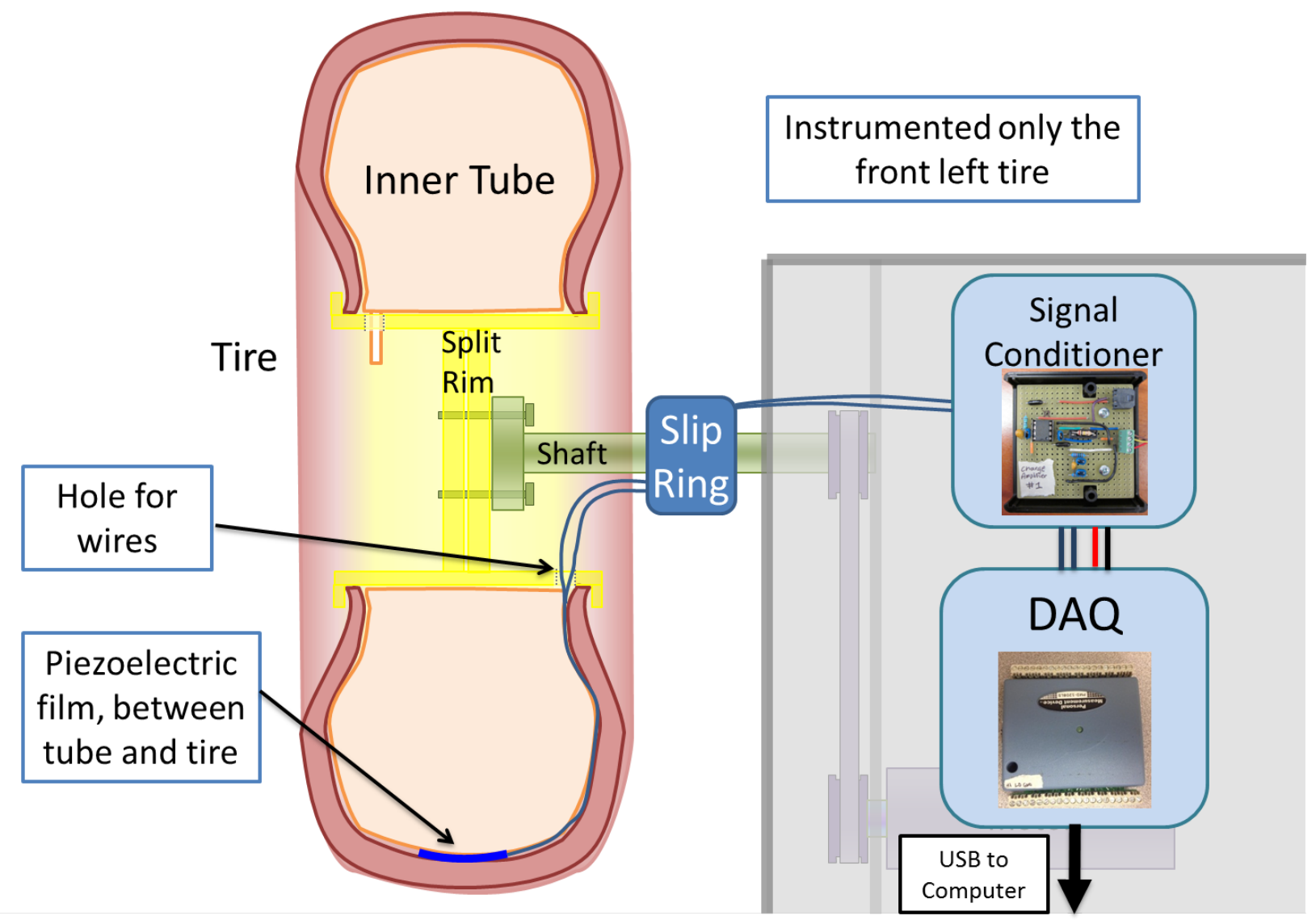

Figure 3.9: Diagram of the tire sensor layout and connections to the on-board electronics.

Table 3.6: Specifications for the SRA-73683-6 slip ring.

\begin{tabular}{lrl}
\hline Parameter & Value & Unit \\
\hline Max speed & 120 & $\mathrm{RPM}$ \\
Max electrical noise at max speed & 50 & $\mathrm{~m} \Omega$ \\
Length & 0.82 & $\mathrm{in}$ \\
Outside diameter & 1.375 & $\mathrm{in}$ \\
Through-bore diameter & 0.5 & in \\
Number of circuits & 6 & \\
Length of leads & 12 & in \\
Voltage & 210 & $\mathrm{~V}$ \\
Current rating, per ring & 2 & $\mathrm{~A}$ \\
Operating temperature & -40 to +80 & ${ }^{\circ} \mathrm{C}$ \\
\hline
\end{tabular}

Source: Moog Components Group [41] 


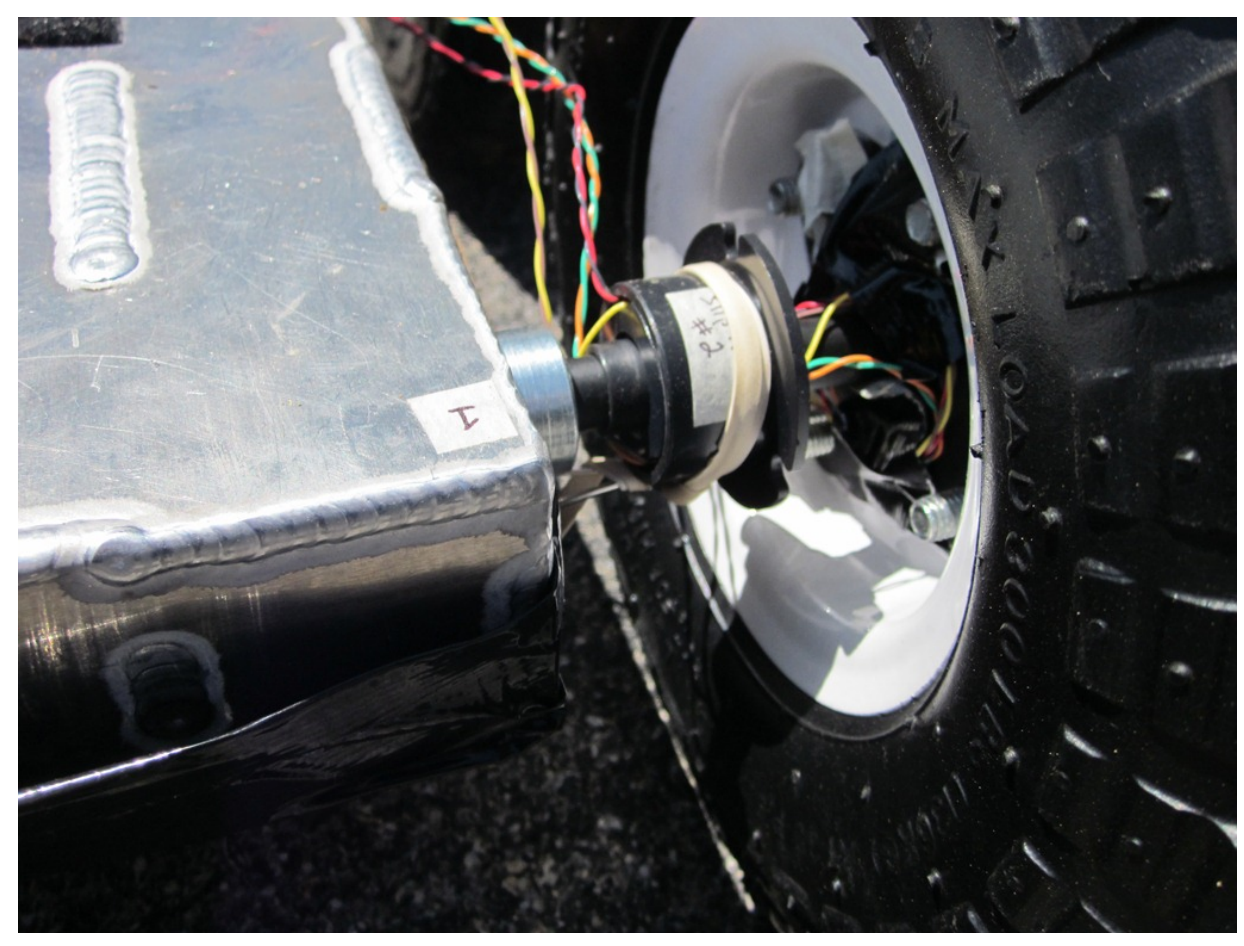

Figure 3.10: Photograph of the slip ring mounted on the axle for the 10" tires.

\subsubsection{Data Acquisition Hardware}

A PMD-1208LS data acquisition (DAQ) unit by Measurement Computing (Norton, MA) was used to acquire signals. The unit used is owned by the Center for Vehicle Systems and Safety (CVeSS) and was lent for this project. The available connections on the PMD-1208LS are shown in Table 3.7.

The $5 \mathrm{~V}$ analog output was used to power the signal conditioning circuit. The signal conditioning circuit used single supply op amps, which mean that that output of the circuit will have a voltage swing centered around $2.5 \mathrm{~V}$. The output voltage could only range from 0 to $5 \mathrm{~V}$, as will be lated explained in Section 3.4.4. The signal of interest was not the voltage level relative to ground, but the difference between $2.5 \mathrm{~V}$ and the output voltage. This meant that the differential input had to be used on the DAQ. When set to operate in differential mode, the allowable input ranges that are within $2.5 \mathrm{~V}$ are $\pm 1 \mathrm{~V}, \pm 1.25 \mathrm{~V}, \pm$ 2 , and $\pm 2.5 \mathrm{~V}[35]$.

To achieve the highest level of resolution relative to the signal size, an input voltage range should be selected so that the signal spans as much of the available voltage as possible. The least significant bit LSB can be used to describe the smallest change of amplitude that can be detected by a DAQ. The LSB can be found from

$$
\mathrm{LSB}=\frac{V_{\max }-V_{\min }}{2^{n}}-1
$$

where $V_{\max }$ and $V_{\max }$ are the maximum and minimum voltage levels that can be read by the 
Table 3.7: Measurement Computing PMD-1208LS DAQ connections.

\begin{aligned} & \hline Quantity Port type \\ & \hline $8^{*}$ Analog input \\ & 2 Analog output \\ & 16 Digital input/output \\ & 1 Power connection $(5 \mathrm{~V}) \\ & 2$ External trigger source \\ & 1 External trigger event counter \\ & 10 Ground connections \\ & 1 Calibration \\ & Source: Measurement Computing Corporation $[35] \\ &$ * can be configured as 4 differential pairs or 8 singles \end{aligned}

DAQ and $n$ is the number of bits. The differential inputs of the PMD-1208LS had 12 bits [35]. Therefore, the LSB for the $\pm 1 \mathrm{~V}$ configuration is $0.00049 \mathrm{~V}$ compared to the $\pm 2.5 \mathrm{~V}$ configuration which has a LSB of $0.00122 \mathrm{~V}$. If the signal of interest had a $0.75 \mathrm{~V}$ voltage swing around $2.5 \mathrm{~V}$, the best resolution would be achieved by using a $\pm 1 \mathrm{~V}$ configuration.

\subsubsection{On-Board Computer}

A computer was necessary to collect data recorded by the DAQ and eventually to implement a feedback loop. While this could be accomplished with a microcomputer, I believed that it would be more straightforward and user friendly to use a laptop. A Fujitsu Lifebook T4215 owned by the Advanced Vehicle Dynamics Lab (AVDL) at Virginia Tech (Blacksburg, VA) was selected because it was available and fit on the robot. The computer features a touch screen that can lay flat on the keyboard, providing stability during motion of the robot. The computer has three USB ports which were used for connections to the motor controller and the DAQ.

\subsubsection{LabView Virtual Instrument}

I designed and created a custom LabView Virtual Instrument (VI) to conduct the tests [45]. The VI is very robust, allowing it to work for many different sensors and settings configurable from the front panel. Through LabView, serial channels are created to communicate with both the DAQ and the motor controller. These connections are shown in Figure 3.8.

After both DAQ and motor controller communication channels are established, a signal is sent to both channels indicating to start the test. The end of data acquisition can be signaled manually by the user or pre-configured after a specified duration of time. Alternatively, if a script is running on the motor controller, the VI will end data acquisition once the script on the motor controller has been completed. The VI front panel is provided in Appendix B. 


\subsection{Signal Conditioning}

Before connecting the leads from the piezoelectric sensors to the DAQ, the signal is passed through a conditioning circuit. There are three purposes of the signal conditioning circuit:

1. convert the charge generated by the piezoelectric sensors to analog voltage to be read by the DAQ

2. ensure that the voltage signal is within the preconfigured input limits of the DAQ

3. filter out noise and unwanted electromagnetic interference

Throughout the design of the signal conditioning circuit, it is important to understand each of the components selected and how the output signal relates to the input signal. In Yi [72], the researchers had a poor understanding of the sensitivity of their signal conditioning circuit which presented difficulties for interpreting their results.

In order to develop a signal conditioning system, the constraints placed on the system by the expected inputs and desired outputs must be established. Then, an analysis of the unconditioned signal must be performed in order to identify the types of conditioning that may be necessary. Those steps are outlined in the following sections.

\subsubsection{Design Constraints for Signal Conditioning System}

Before designing the signal conditioning circuit, the parameters that limit the input and output of the signal conditioner were established.

The $+5 \mathrm{~V}$ analog output and ground connection on the DAQ, described in Section 3.3.2, were used to power the circuit. The user manual for the DAQ recommends that the current drawn from the $+5 \mathrm{~V}$ source be less than 300-320 mA[35]. Therefore, one requirement is that the signal conditioner must operate with connections to only $+5 \mathrm{~V}$ and ground and must draw less than $300 \mathrm{~mA}$ of current.

As was explained in Section 3.3.2, the size of the analog voltage output of the signal conditioner should correspond to the allowable input range of the DAQ. The signal that is output by the signal conditioner must be within an allowable differential voltage input range for the DAQ. In order to maximize the resolution relative to the signal strength, the signal should span as much of the input range possible without exceeding the range. The signal strength from the piezoelectric sensors is expected to be different for different sensor types and for the same sensors at different locations and under different conditions. In order for all sensors to span the voltage input range, the gain of the signal conditioner should be adjustable.

The signal conditioner should be able to be located on the non-rotating robot chassis. Recall that the signal from the sensors, located on the rotating tires, is transferred through the slip ring to the DAQ, located on the non-rotating robot. Due to the small size of the tires, there may be limited space on the tire for a signal conditioning circuit and even a small printed circuit board may disturb the inertial balance of the wheel and affect the system 
dynamics. Placing the signal conditioner on the robot will require longer wires between the sensors and the conditioner. This introduces the requirement that the conditioner should not be affected by the capacitance of these wires or electromagnetic interference picked up by these wires.

The intent of this project is not to create a one-time-only prototype that cannot be replicated. Instead, the intent is to create a system that can be widely used and replicated. Therefore, any components selected for the system should be affordable and readily accessible to the general public. Furthermore, any circuit components should be available in both surface mount and through hole configurations so that the same circuit can be prototyped on a breadboard or prototype board and later be implemented as a printed circuit board.

The signal conditioner should amplify the frequency range that contains the signals of interest from the piezoelectric sensors and attenuate noise and unwanted signals from the motors and other on-board electronics. An analysis of the expected frequency and strength of the input signal is presented in Section 3.4.3. In Section 3.4.2, sources of noise and electromagnetic interference are investigated. The frequency ranges of the interference should be attenuated by the signal conditioner.

\subsubsection{Identification and Reduction Electromagnetic Interference}

When developing a signal conditioning circuit for a piezoelectric sensor, it is important to identify and reduce or eliminate sources of noise and unwanted electromagnetic interference. To assist with this process, the frequency spectrum of the signal can be examined.

\section{Types of Electromagnetic Interference}

Noise and unwanted interference typically falls into one of three categories:

- white (broadband) noise

- 1 /f noise

- specific frequency

White noise is typically from two sources: thermal/Johnson noise and shot noise. Thermal/Johnson noise comes from a resister and can be minimized by using smaller resistances in a signal conditioning circuit $[9,31,64]$. Shot noise is typically much smaller than Johnson noise. It originates from small variations in electric current across vacuum tubes and transistors. Shot noise is minimized by minimizing the number of these voltage potential barriers [64].

Other noise sources have a spectral density that decreases with increasing frequency. One name for this is contact noise. Contact noise occurs across joints in electrical circuits, like solder joints. The magnitude of the noise is proportional to the DC current across any contact [64]. 
While white noise and 1/f noise are from the internal workings of the circuit, unwanted frequencies can also occur due to interference from an external electromagnetic field. This interference appears as a specific frequency in the power spectrum. This happens when either a conductor moves in a constant magnetic field or there is a changing magnetic field near a conductor. There are many physical ways in which electromagnetic noise can be reduced, including using shielded wires, twisting the wires into pairs, or placing the circuit in a Faraday cage [64]. Electromagnetic interference can also be filtered out of the circuit, as long as the frequency of the interference is not the same as the signal of interest. Ground loops can also cause noise in a circuit.

\section{Examination of Noise In Test System}

In order to maximize the output signal amplitude and minimize noise and electrical interference, the sources and characteristics of the noise and interference in the robot test system were identified. Using different configurations of the components, plots of the power spectral density of the voltage signal(s) measured by the DAQ were examined for frequency characteristics. The results from these tests are summarized in Table 3.8. In this section, the various sources of interference and methods to reduce or eliminate them are examined.

Table 3.8: Possible noise sources to identify how to reduce them and what frequencies the conditioner should attenuate.

\begin{tabular}{llll}
\hline Source & White noise & $1 /$ f noise & Specific frequency \\
\hline $\begin{array}{l}\text { Computer connected to wall } \\
\quad \text { no }\end{array}$ & no & $60 \mathrm{~Hz}$ \\
Motors running & no & no & $120 \mathrm{~Hz}$ multiples \\
USB to motor controller & no & yes & $30 \mathrm{~Hz}$, decreases motor noise \\
Slip ring & no & no & picks up more motor noise \\
\hline
\end{tabular}

It was found that connecting the computer to the wall power outlet introduced a 60 $\mathrm{Hz}$ signal, which is the power line frequency in North America. This can be remedied by ensuring that the computer is not connected to an electrical outlet during testing.

When the USB connection between the computer and the motor controller was disconnected, $1 /$ f noise appeared in the spectral density plot as well as a signal at approximately 30 Hz. The source of this noise is not known, but I can speculate that connecting another USB port to the motor controller, which is connected to a battery, may eliminate some sort of ground loop. Nonetheless, this phenomena is a benefit because during testing, the computer will always be connected to the motor controller. If any future studies with this equipment do not use a wired connection between the computer and the motor controller, this $30 \mathrm{~Hz}$ signal will need to be addressed.

When the motors were running, a strong signal was seen at integer multiples of $120 \mathrm{~Hz}$. This $120 \mathrm{~Hz}$ signal was stronger when the slip ring was attached to the DAQ compared 
to no wire connections in the DAQ probably because the wires of the slip ring were also picking up the signal. In a study examining noise sources in measurements by a strain gauge, Yüksekkaya et al. [76] found that noise from the small electric motor was reaching the measurement system through attached wires. In our test system, the wired route from the motors to the computer is via the motor controller. I do not believe that this connection is causing the $120 \mathrm{~Hz}$ signal because it was found that connecting the USB cable from the computer to the motor controller reduced the $120 \mathrm{~Hz}$ signal rather than increased it. This suggests that the signal is due to electromagnetic interference. While twisting wires in pairs and placing them in a shield may help to reduce this interference, I do not expect that it can be eliminated by mechanical measures alone. The DAQ itself picked up this interference even when no wires were attached. In order to reduce this effect, the signal conditioning circuit should attenuate $120 \mathrm{~Hz}$ signals and the signal conditioner should be placed as close as possible to the DAQ.

There was some concern that the slip rings may introduce noise while rotating. The presence of noise from the rotation of the slip rings was not seen. Because any tests with the rotating slip ring also had the motors running, any noise, if present, may have been overshadowed by the interference from the motor.

\subsubsection{Evaluation of Input Signal Strength and Frequency Range}

To determine the expected size of the signal to be conditioned, unconditioned signals from the piezoelectric sensors were measured directly by the DAQ. Because of the mismatch between the impedance of the DAQ and the impedance of the piezoelectric sensors, the low frequency signal was attenuated. The analysis performed to determine the original signal strength is provided here.

When the voltage drop over a capacitor is measured with a voltmeter or DAQ, the circuit acts as a voltage divider with output voltage

$$
V_{\text {out }}=V_{C} \frac{Z_{R}}{Z_{R}+Z_{C}}
$$

where $V_{C}$ is the actual voltage drop over the capacitor, $Z_{r}$ is the internal impedance of the measuring device, and $Z_{C}$ is the impedance of the capacitor. Voltmeters are designed for situations where $Z_{R} \gg Z_{C}$ so $V_{\text {out }} \approx V_{C}$. As $Z_{C}$ approaches $Z_{R}$, the measured signal is attenuated.

The concept illustrated above applies to this study because the piezoelectric film sensors act as capacitors as described in Section 2.2.4. The electrical impedance $Z_{C}$ of a piezoelectric sensor with capacitance $C_{p}$ is

$$
Z_{C}=\frac{1}{j \omega C_{p}}
$$

where $\omega$ is the angular frequency and $j$ is imaginary. The relation between frequency $\omega$ $(\mathrm{rad} / \mathrm{s})$ and frequency $f(\mathrm{~Hz})$ is

$$
f=2 \pi \omega .
$$


The electrical impedance of the measuring device depends on its internal resistance. The electrical impedance $Z_{R}$ of a DAQ with resistance $R$ is

$$
Z_{R}=R_{\text {meter }}
$$

Substituting Equations 3.4-3.6 into Equation 3.3 gives

$$
V_{\text {out }}=V_{p} \frac{R_{\text {meter }}}{R_{\text {meter }}+\frac{1}{2 \pi f C_{p}}}
$$

where $V_{p}$ is the voltage across the piezoelectric sensor, and $R_{\text {meter }}$ is the internal resistance of the measuring device.

Because the capacitance of the piezoelectric sensors is so small ( 0.74 to $2.78 \mathrm{nF}$, as given in Table 3.5), the internal resistance of the measuring device must be considered. According to Measurement Computing Corporation [36], the internal impedance of most DAQ input channels is $1 \mathrm{M} \Omega$ or higher. This means that as the frequency increases, the measured voltage output approaches the true voltage output of the piezo.

After conducting a preliminary analysis, it was determined that the target gain should be on the order of $60-70 \mathrm{~V} / \mu \mathrm{C}$ or approximately $0.1 \mathrm{~V} / \mathrm{V}$ for $C_{p}=1.38 \mathrm{nF}$.

The slip rings allow a maximum rotational frequency of 120 RPM (Table 3.6). This is similar to the rated speed of our motors which is 122 RPM (Table 3.3). If our piezo films pick up a signal every rotation of the wheel, then we can expect a signal to appear at a maximum frequency of $2 \mathrm{~Hz}$.

\subsubsection{Design of Signal Conditioning Circuit}

In the Sections 3.4.1-3.4.3 the constraints and goals of the signal conditioning circuit were defined. In this section, the design of the signal conditioning circuit itself is discussed.

The first purpose of the signal conditioning circuit is to convert from charge to voltage. This can be accomplished with a charge amplifier circuit, like that shown in Figure 3.11 $[9,30,37]$. The charge amplifier functions to remove charge accumulated on the faces on the electrodes surrounding the piezoelectric material and convert it into a voltage signal. The output seen by the measuring device has a low impedance compared to the high impedance of the sensor. This prevents the signal from being attenuated due to impedance mismatch with the measuring device.

The charge amplifier acts as a high pass filter with cutoff frequency

$$
f_{h p f 1}=\frac{1}{2 \pi R_{f b} C_{f b}}
$$

where $R_{f b}$ and $C_{f b}$ correspond to the resistance and capacitance shown in Figure 3.11. The resistor $R_{\text {in }}$ is not necessary for the charge amplification, however it was added in order to create a low pass filter to reduce the interference from the motors. The resulting low pass filter created has a cutoff frequency of

$$
f_{l p f 1}=\frac{1}{2 \pi R_{i n} C_{p}}
$$




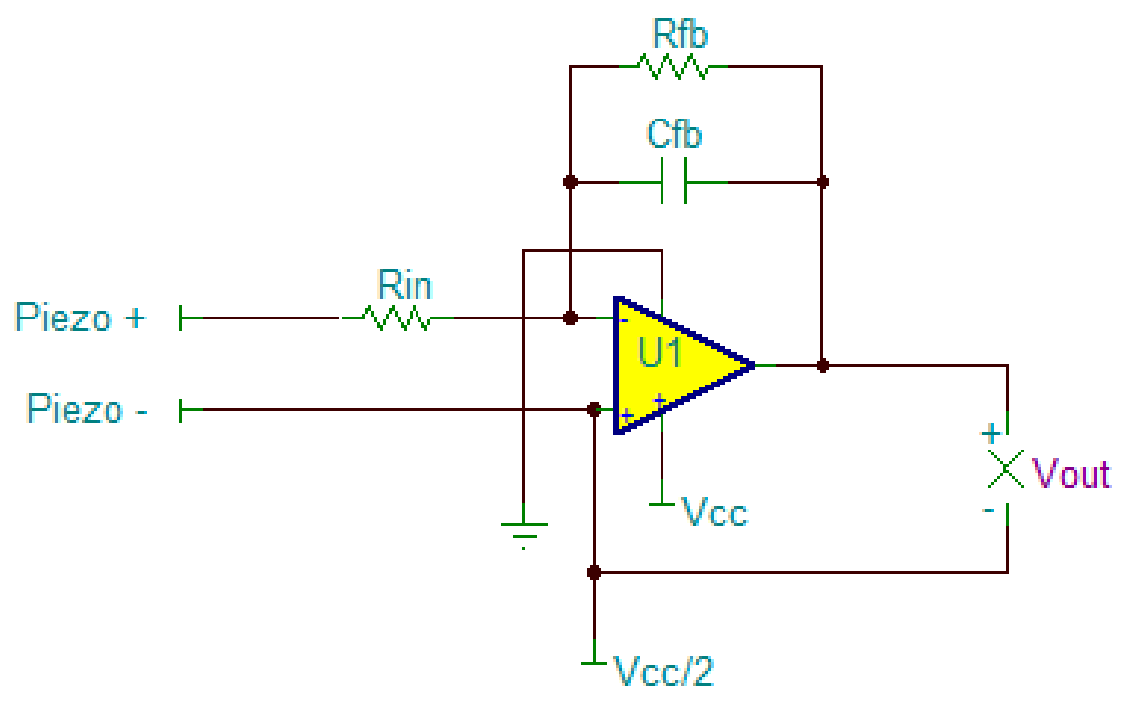

Figure 3.11: Circuit diagram of a charge amplifier, powered by a single supply op amp.

where $C_{p}$ is the capacitance of the piezoelectric sensor. The gain of the charge amplifier is characterized as the output voltage as a fraction of charge on the piezoelectric sensor. For the basic charge amplifier in Figure 3.11, the gain is

$$
A_{1}=\frac{1}{C_{f b}}\left[\frac{\mathrm{V}}{\mathrm{C}}\right] .
$$

The charge amplifier in Figure 3.11 is operated with a single supply operational amplifier (op amp). For this type of op amp, the output voltage can only exist between ground and the supply voltage. In order to measure both negative and positive signals, the non-inverting input is connected to a voltage level of half the supply voltage. The result is that the output signal swings around the half-supply level.

Many challenges arise when selecting resistor and capacitor values for a charge amplifier [9] for a piezoelectric film sensor. In order to get the required gain shown in Equation 3.10, the feedback capacitor, $C_{f b}$ must be small. As $C_{f b}$ is decreased, the cutoff frequency of the effective high pass filter described in Equation 3.8 increases. In order to use the piezo film to measure strain that changes slowly with time, $f_{h p f 1}$ must be kept low. To keep $f_{h p f 1}$ low, even when $C_{f b}$ is very small, $R_{f b}$ must be very large. When very large resisters are used with an op-amp, the intrinsic noise of the op amp can be amplified. Furthermore, very large resistors (hundreds of $\mathrm{M} \Omega$ ) can be expensive to purchase and are often low-precision.

One of the design constraints explained in Section 3.4.1 was that the gain of the signal conditioner should be adjustable. Using just a charge amplifier, $C_{f b}$ would be adjusted to change the gain. This would also result in a change to $f_{h p f 1}$, which may complicate an analysis.To address this design challenges introduced by use of a charge amplifier, the signal conditioning was divided into two stages [57]. 


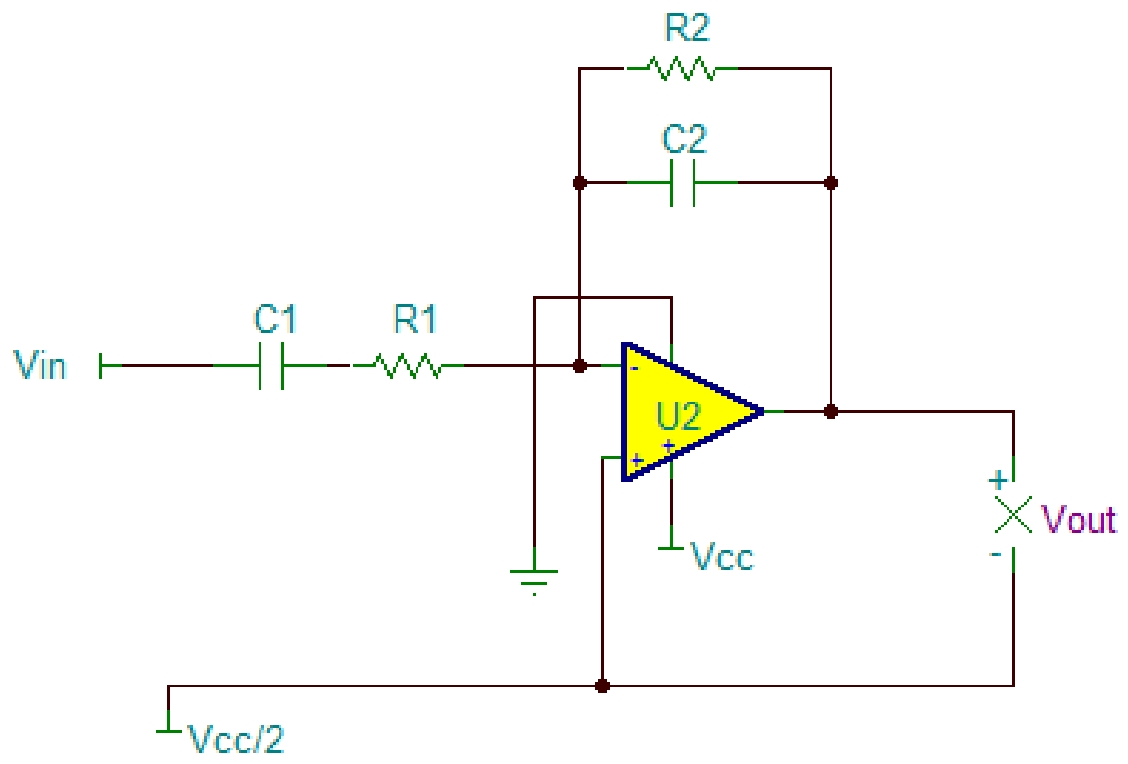

Figure 3.12: Circuit diagram of an inverting amplifier, powered by a single supply op amp.

In the first stage, the charge was transferred to voltage, using a charge amplifier as seen in Figure 3.11. The second stage of the signal conditioner consisted of an inverting amplifier, shown in 3.12. The combined circuit with the component values is shown in 3.13. The low pass frequency for the inverting amplifier is

$$
f_{l p f 2}=\frac{1}{2 \pi R_{2} C_{2}}
$$

while the high pass frequency is

$$
f_{h p f 2}=\frac{1}{2 \pi R_{1} C_{1}}
$$

The gain of the amplifier is

$$
A_{2}=\frac{-R_{2}}{R_{1}}\left[\frac{\mathrm{V}}{\mathrm{V}}\right]
$$

In order to modify the gain of the circuit without changing $f_{h p f 2}, R_{2}$ can be adjusted. While changes to $R_{2}$ will affect $f_{l p f}$, the purpose of the low pass filter is to reduce higher frequency interference. It is not expected that changes to $f_{l p f 2}$ will result in significant changes to the strength of the low frequency signal of interest.

When the gains in Equations 3.10 and 3.10 are combined, the resulting gain for the combined circuit shown in Figure 3.13 is

$$
A=\frac{-R_{2}}{R_{1} C_{f b}}\left[\frac{\mathrm{V}}{\mathrm{C}}\right]
$$




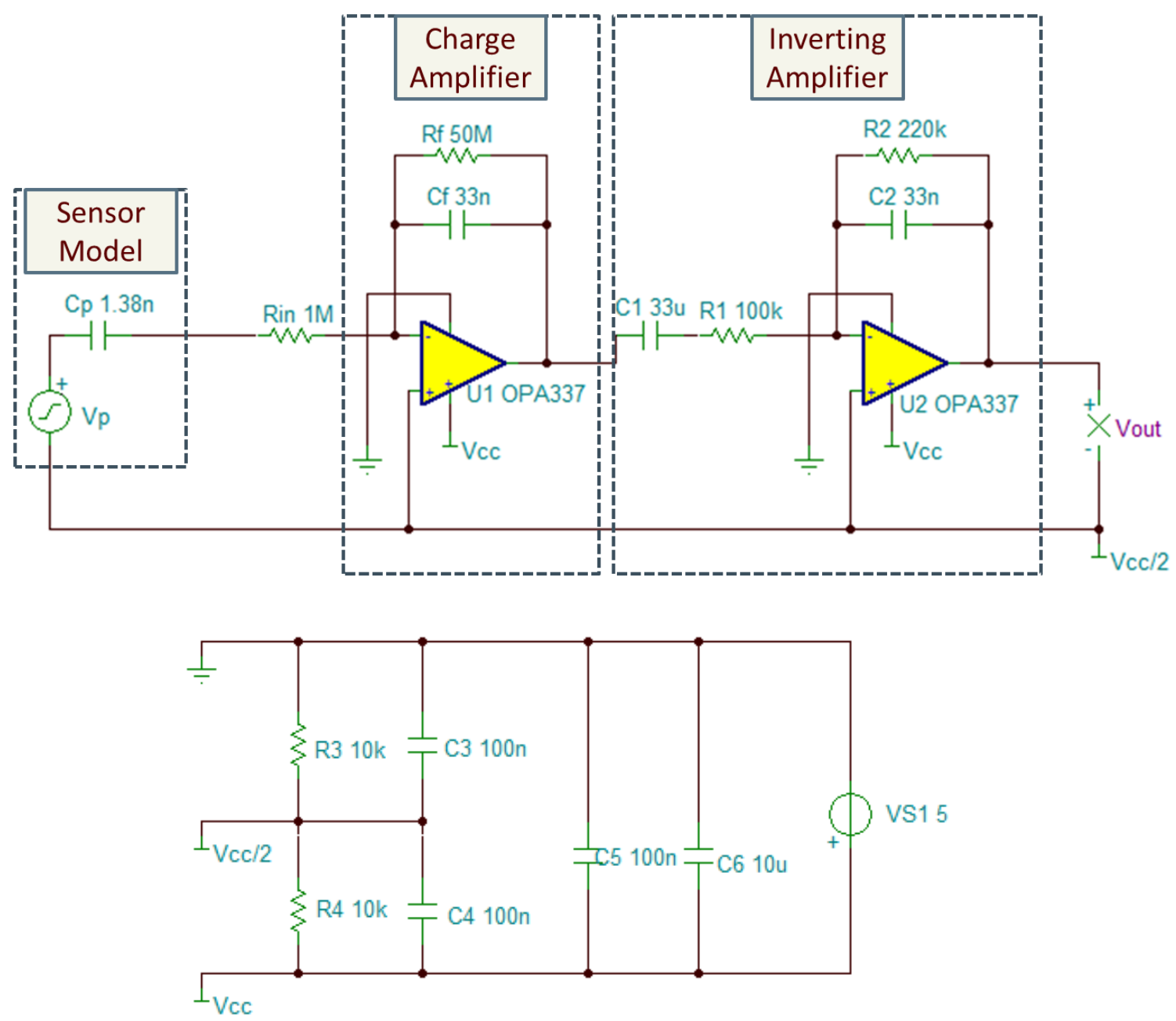

Figure 3.13: Circuit diagram for the two stage signal conditioning circuit. 

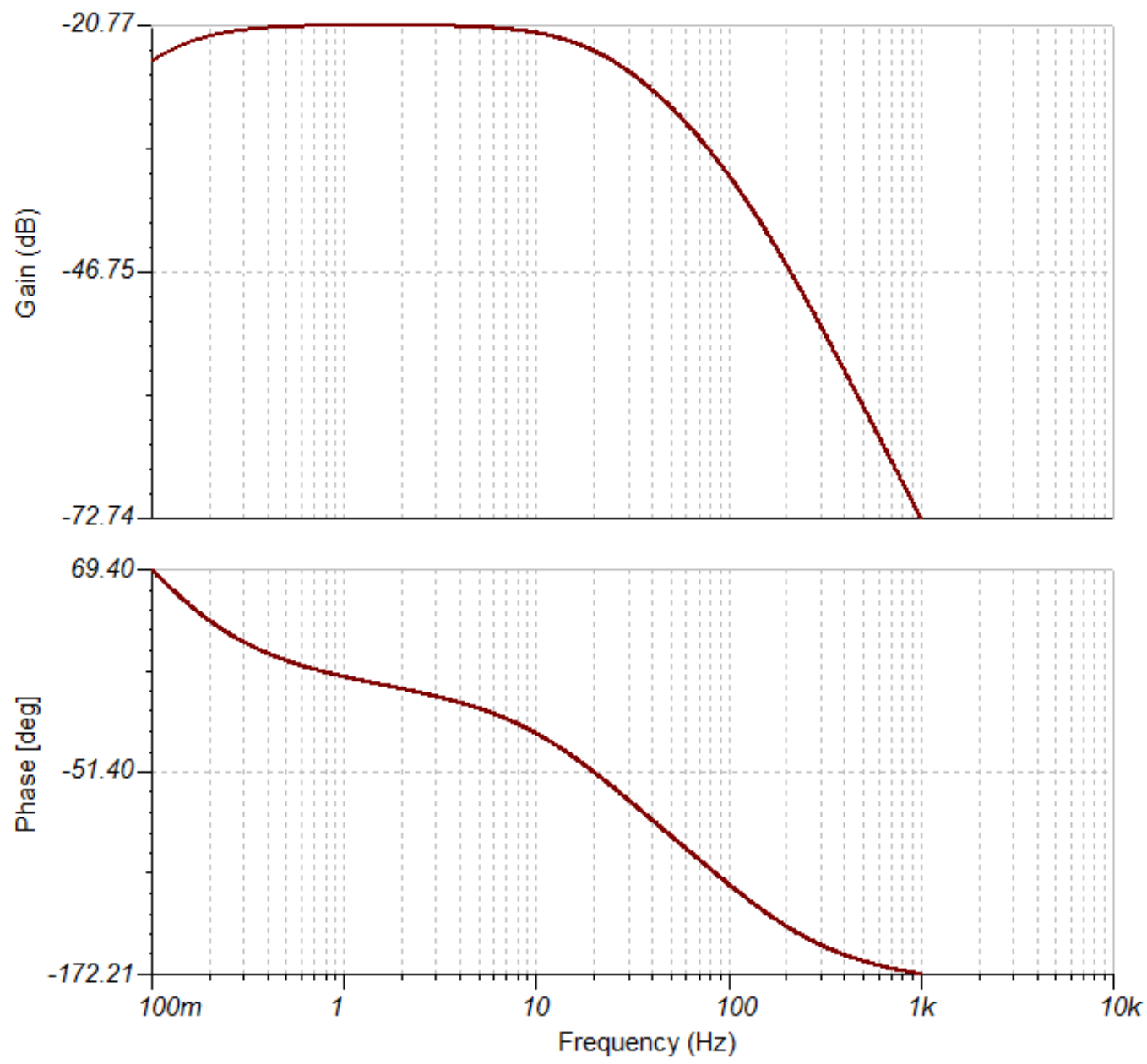

Figure 3.14: Transfer function for signal conditioner, created in TINA-TI. 
The gain and phase diagram for the signal conditioner are shown in Figure 3.14.

In Figure 3.13, the sensor is modeled as a voltage source $V_{p}$ connected in series with a capacitor $C_{p}$. In relative, the mechanical input to the piezoelectric sensor is what creates a voltage difference across the effective capacitor. Also in Figure 3.13, $5 \mathrm{~V}$ analog input from the DAQ is shown as a $V S$. In order to center the output around $2.5 \mathrm{~V}$ as was previously discussed, a voltage divider was created with $R_{3}$ and $R-4$. Capacitors $C_{3}, C_{4}, C_{5}$, and $C_{6}$ are used to reduce noise in the power supply. The differential output that is measured by the DAQ is labeled as $V_{\text {out }}$.

\subsubsection{Operational Amplifier Selection}

With a huge selection of operational amplifiers (op amps) available on the market, some thought had to be put into selecting one that would work within our target parameters and would maintain a high signal-to-noise ratio. In order to be directly powered by the $5 \mathrm{~V}$ output on the DAQ, the operational amplifier must be powered by a single $5 \mathrm{~V}$ supply. To have flexibility to prototype any conditioning circuits on a breadboard and then to later create a printed circuit board of the same circuit, the selected op amp should be available in both through hole and surface mount configurations. Because the standard package is a 8 pin surface mount, space on the prototype board will be optimized by using a dual op amp package. When using dual op amp packages, it should be verified that there is not crosstalk between the amplifiers.

The large impedance of the piezoelectric films elevates concerns about noise from the op amp. Because any input bias (quiescent) current will also be amplified by the circuit, the input bias current should be small. The input resistance should also be very high so that it will match or exceed the high impedance of the sensor. Both of these goals can be achieved by looking at precision op amps. In case we want to use the same op amp as a voltage buffer, the op amp should be unity gain stable. A single supply op amp with rail-to-rail output should have output voltage swings that are within $100 \mathrm{mV}$ of each supply rail [7]. This is desirable for our application.

The company Texas Instruments (Dallas, TX) was selected because of their wide selection and in depth technical support available on their website [68]. Texas Instruments also offers a free circuit modeling software, TINA-TI, which allows for modeling of specific Texas Instruments components. After applying the considerations described above, the op amps OPA 337 and OPA 350 were selected as the best candidates [66, 67]. In the end, OPA337 was selected because of its lower noise at low frequencies and because it is used and analyzed extensively in Bartolome [9].

\subsubsection{Construction of Signal Conditioning Circuit}

The signal conditioner was constructed on a $2.5 " \times 2.5 "$ prototype board. Components were purchased for through-hole configurations and hand soldered to the board. The box came with a convenient enclosure to protect the circuit. The prototype circuit is shown in Figure 3.15. The resistor $R_{2}$ was put into a mount so that it could be changed if the desired 
gain needed to be altered. Two identical boards were made. The connector selected for attachment of the slip ring leads to the circuit board is indicated in Appendix A.

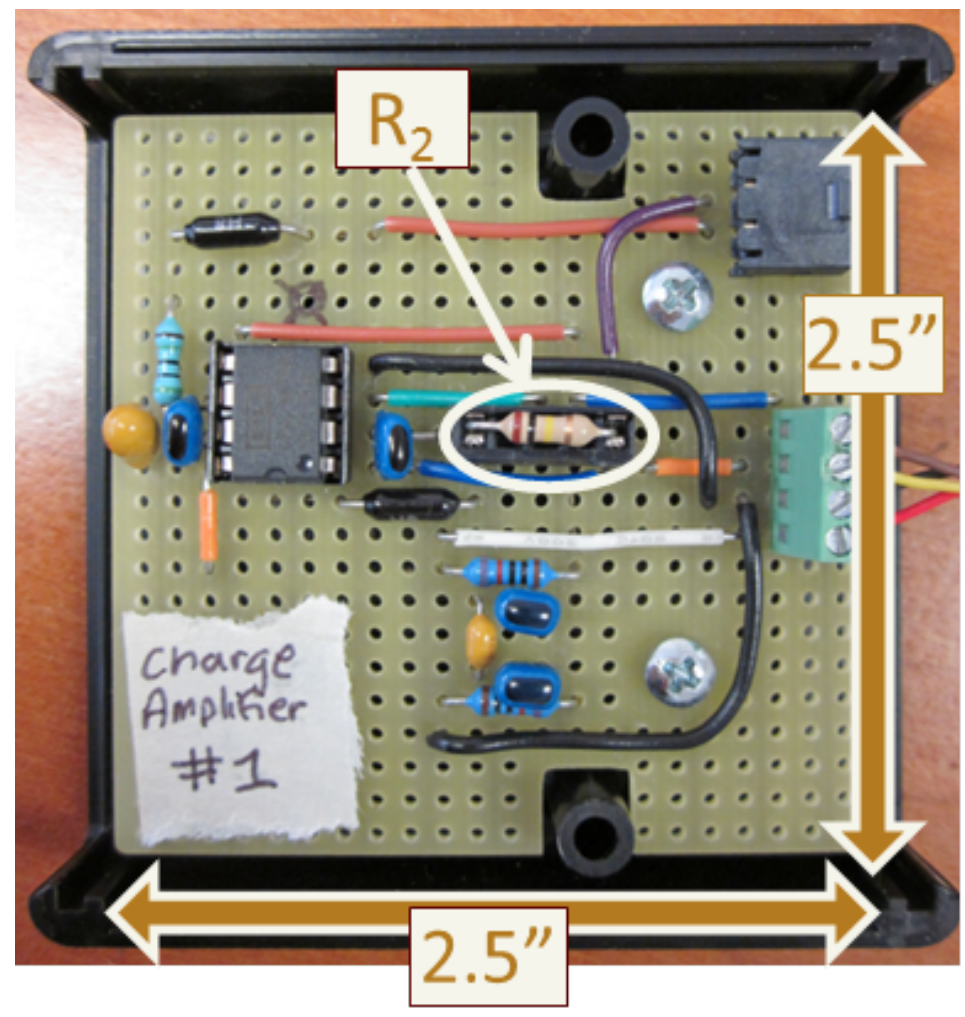

Figure 3.15: Signal conditioner created on a prototype board, placed in an enclosure.

The requirements laid out in Section 3.4.1 were all met. The circuit was successfully constructed using inexpensive, readily available components. The circuit can be attached to the DAQ and powered by only a $+5 \mathrm{~V}$ and ground input. The gain of the circuit is adjustable. Noise from the motor at $125 \mathrm{~Hz}$ is filtered out and the lower frequency signals of interest are amplified. For the circuit values in Figure 3.15, the gain of the circuit calculated from Equation 3.14 is about $67 \mathrm{~V} / \mu \mathrm{C}$, which is consist with the target established in Section 3.4.3.

\subsection{Review of Design of Test System}

In this chapter, the steps of the design process for the robot test system were presented. A robot was successfully selected and constructed that met basic requirements of the study. One tire for each of the two tire sizes was instrumented with a set of piezoelectric film sensors. The signal from the sensors is transferred through a slip ring mounted to the wheel axle of the robot to a signal conditioner, a DAQ, and finally a computer that rides on-board the robot. All of these systems are able to communicate with one another and work together 
effectively. The signal conditioning circuit was designed to extend the time constant of the piezoelectric film sensor and make the output readable by the DAQ. In the next chapter, the methodology designed to test the system is presented. 


\section{Chapter 4}

\section{Testing Methodology}

The third objective of this study was to develop and implement a test procedure for using the robot with the sensors on off-road surfaces. In this section, the testing methodology developed is reviewed. First, the five parameters that make up the test matrix are explained. The preparation and methods of characterization of the test surface are reviewed in Section 4.2. The measurements taken during testing via the motor controller and the DAQ are explained in Section 4.3. Finally, in Section 4.4, the steps of data processing are described.

\subsection{Test Parameters}

In order to investigate the ability of the piezoelectric sensors in the robotic tires to evaluate ground and vehicle characteristics, a series of tests were performed. The test matrix consisted of the following variables:

1. Tire size

2. Applied power

3. Payload

4. Ground surface

5. Sensor type and location

Test parameters 2-3 were selected (1) to determine the sensitivity of the signal recorded by the sensors to changes in these parameters and (2) to determine if differences in ground characteristics could be better detected with a configuration.

Each of the test parameters is described in greater detail in the following sections. 


\subsubsection{Tire Size}

All tests were conducted using the 10" diameter tires and 6" diameter tires that are described and pictured in Section 3.1.5. All tires were kept at an inflation pressure of approximately 26 psi. There were some differences in the testing protocols for the two tire sizes.

At a given speed, the robot would travel further per unit time with the 10" tires than with the 6" tires, owing to the different diameters. In order to prevent the robot from traveling off of the prepared terrain (particularly the ice surface described in Section 4.1.4), the time duration of each test was reduced for the 10" tires. This is explained in more detail in Section 4.1.2. The 10" tires also had a larger mass than the 6" tires, making the static weight of the robot larger. This is described in Section 4.1.3.

Attempts were made to have the sensor locations be consistent for both tires, although in practice, this was not the case. The specific locations of the sensors and how they varied for each tire is described in Section 4.1.5.

\subsubsection{Applied Power}

The robot was run at three power levels: $50 \%, 75 \%$ and $100 \%$ of the maximum power available. This is similar to the power levels tested by Johnson [29].

The power distributed to the robot is determined from the basic equation for power,

$$
P=V I
$$

where $\mathrm{P}$ is power, $\mathrm{V}$ is voltage, and $\mathrm{I}$ is current. The maximum current for the robot was set to $20 \mathrm{~A}$ and the voltage was provided by a $24 \mathrm{~V}$ battery. At the beginning of each test, the controller reported to the computer the available battery voltage which could vary slightly throughout use. In open loop power mode, if the robot encounters resistance to motion (for example due to an obstacle or a change in load), the speed will decrease.

The sequence of power given to the motors for each test was controlled by a custom MicroBasic script, preloaded onto the controller. The scripts used for the 6" and 10" tires are provided in Appendices $\mathrm{C}$ and D, respectively. During the test, the LabView VI would send a serial command to start the script and indicate which power level to use. The script consisted of a specific sequence of commands for each test:

1. Begin at rest (0\% power).

2. Apply $P(\%)$ power for time $T(\mathrm{~s})$.

3. Apply $0 \%$ power for $8 \mathrm{~s}$.

4. Apply $-P(\%)$ power for time $T(\mathrm{~s})$, returning the robot to its approximate starting position.

5. Apply 0\% power.

The approximate distance the robot would travel during the test was determined by the power level $\mathrm{P}$, the duration $\mathrm{T}$ of the applied power, and the diameter of the wheels. The 
Table 4.1: Details of the programmed power sequences for each tire size and applied power.

\begin{tabular}{lcc}
\hline & \multicolumn{2}{c}{ Tire diameter } \\
\cline { 2 - 3 } & $6 "$ & $10 "$ \\
\hline Duration of applied power, T $(\mathrm{s})$ & & \\
$\mathrm{P}=50$ & 12.5 & 7 \\
$\mathrm{P}=75$ & 7 & 4 \\
$\mathrm{P}=100$ & 5 & 3 \\
Max rate of power change $\left(\frac{\% \text { power }}{s}\right)$ & \pm 50 & \pm 100 \\
\hline
\end{tabular}

ice surface had a limited amount of length available for testing. In order to ensure that the robot would remain on the ice surface, $\mathrm{T}$ and the maximum rate of change of power for each tire and power combination was varied and is shown in Table 4.1. The values described in Table 4.1 applied to all testing surfaces. The rate of power change was higher for the 10" tires in order to guarantee at least one full revolution of the wheel at constant velocity while remaining within the distance constraints.

\subsubsection{Payload}

Tests were conducted under two load conditions: (1) no added load and (2) applied $10 \mathrm{lb}$ $(4.54 \mathrm{~kg})$ weight, placed on the vehicle platform under the laptop computer, as shown in Figure 4.1. Because the $10 \mathrm{lb}$ weight was not added in the center of the vehicle, the added weight was not evenly distributed across the wheels. The load on each wheel for each tire and weight configuration was measured using an approximation technique.

Measurements were taken on a Acculab SVI-100E, which has a $100 \mathrm{~kg}$ capacity and 0.02 $\mathrm{kg}$ resolution. The scale was leveled and the accuracy was checked with a weight with a known $50 \mathrm{lb}(22.68 \mathrm{~kg})$ weight. First, the total weight of the robot $W_{\text {total }}$ was measured. Next, the wheels were measured in four pairs: $W_{\text {front }}, W_{\text {rear }}, W_{\text {left }}$, and $W_{\text {right }}$. In order to accomplish this, a wooden block approximately the same height as the scale was placed next to the scale. To measure $W_{\text {front }}$ for example, the front right and front left tires were placed on the scale and the rear left and rear right tires were placed on the wooden block. This method was repeated and the results recorded for the other pairs.

In practice, the sum of $W_{\text {front }}$ and $W_{\text {rear }}$ and the sum of $W_{\text {left }}$ and $W_{\text {right }}$ vary slightly from $W_{\text {total }}$. This is due to the scale and the wooden block not being perfectly level with one another. To make up for this, the weights are adjusted so that the sums add up to the measured $W_{\text {total }}$. The adjusted weights are 


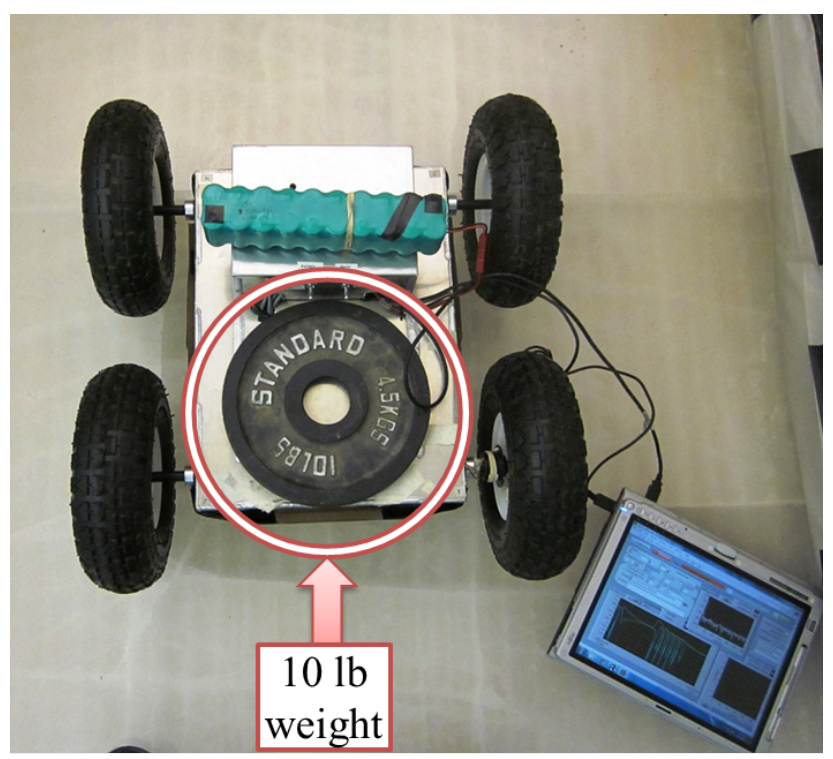

Figure 4.1: Location of $10 \mathrm{lb}$ weight placed under the laptop computer.

$$
\begin{aligned}
W_{\text {front }}^{*} & =W_{\text {front }} \frac{W_{\text {front }}+W_{\text {rear }}}{W_{\text {total }}} \\
W_{\text {rear }}^{*} & =W_{\text {rear }} \frac{W_{\text {front }}+W_{\text {rear }}}{W_{\text {total }}} \\
W_{\text {left }}^{*} & =W_{\text {left }} \frac{W_{\text {left }}+W_{\text {right }}}{W_{\text {total }}} \\
W_{\text {right }}^{*} & =W_{\text {right }} \frac{W_{\text {left }}+W_{\text {right }}}{W_{\text {total }}}
\end{aligned}
$$

where $^{*}$ denotes the adjusted value.

Next, an assumption is made that the differences between $W_{\text {front }}^{*}$ and $W_{\text {rear }}^{*}$ are evenly distributed across the left and right sides. The resulting equations are

$$
\begin{aligned}
W_{F L} & =W_{\text {front }}^{*} \frac{W_{\text {left }}^{*}}{W_{\text {total }}} \\
W_{F R} & =W_{\text {front }}^{*} \frac{W_{\text {right }}^{*}}{W_{\text {total }}} \\
W_{R L} & =W_{\text {rear }}^{*} \frac{W_{\text {left }}^{*}}{W_{\text {total }}} \\
W_{R R} & =W_{\text {rear }}^{*} \frac{W_{\text {right }}^{*}}{W_{\text {total }}} .
\end{aligned}
$$

This method was found to be easier to implement than putting each of the tires individually on the scale. 
These measurements were made for the 6" and 10" tires with and without the added 10 lb weight. The results are given in Section 5.2.1.

\subsubsection{Surfaces}

Testing was carried out on three surfaces: ice, polished cement, and sand. Figure 4.2 shows photographs of the robot on each of the test surfaces.

The two hard surfaces (ice and polished cement) were selected because while both have a smooth texture, they have different coefficients of friction. Both of these surfaces were available at CVeSS at Virginia Tech (Blacksburg, VA). The polished cement was available as the floor of the lab workspace at CVeSS. It is the same cement surface that was tested by Johnson [29]. The ice was prepared as part of an ongoing study in the AVDL, housed within CVeSS. Surface preparation for the two hard surfaces is described in Section 4.2.1. The static coefficient of friction was measured as described in Section 4.2.2.

The sand surface used for testing was on an outdoor recreational sand volleyball court in Blacksburg, VA. Terrain preparation is described in Section 4.2.3. Testing was completed across two days in May 2012. Each day, the cone index was measured using the methodology described in Section 4.2.4.

\subsubsection{Piezoelectric Sensors}

The three locations and types of piezoelectric sensors and the reasons for their selection were previously described in Section 3.2. The configurations are reviewed Table 4.2.

The design of the signal conditioning circuit was described in Section 3.4.4. Preliminary testing revealed that the typical signal from sensor 1 had about twice the amplitude of the signals from sensors 2 and 3 . The amplifier gain of the signal conditioning circuit can be adjusted by changing resistor $R_{2}$, shown in Figures 3.13 and 3.15. The gain of the amplifier was therefore adjusted so that the signals would have similar amplitudes, allowing both signals to take advantage of the full input range of the DAQ.

A piece of Scotch Removable Double Sided Tape was attached to each sensor on the side facing the tire. The wire leads on the piezoelectric sensor were connected to the slip ring, described in Section 3.3.1. The slip ring has six wires, which limits the number of sensors connected to it at a single time to three.

Table 4.2: Piezoelectric sensors and their locations used in testing

\begin{tabular}{llllr}
\hline$\#$ & Type & Lamination & Location & $R_{2}(\mathrm{k} \Omega)$ \\
\hline 1 & DT1-028K/L w/rivets & Unlaminated & Circumferential, along tread & 100 \\
2 & DT1-028K/L w/rivets & Unlaminated & Radial, inner sidewall & 220 \\
3 & LDT1-028K/L w/rivets & Laminated & Circumferential, along tread & 220 \\
\hline
\end{tabular}

Note:\# is for reference within this paper and does not refer to a specific sensor. 


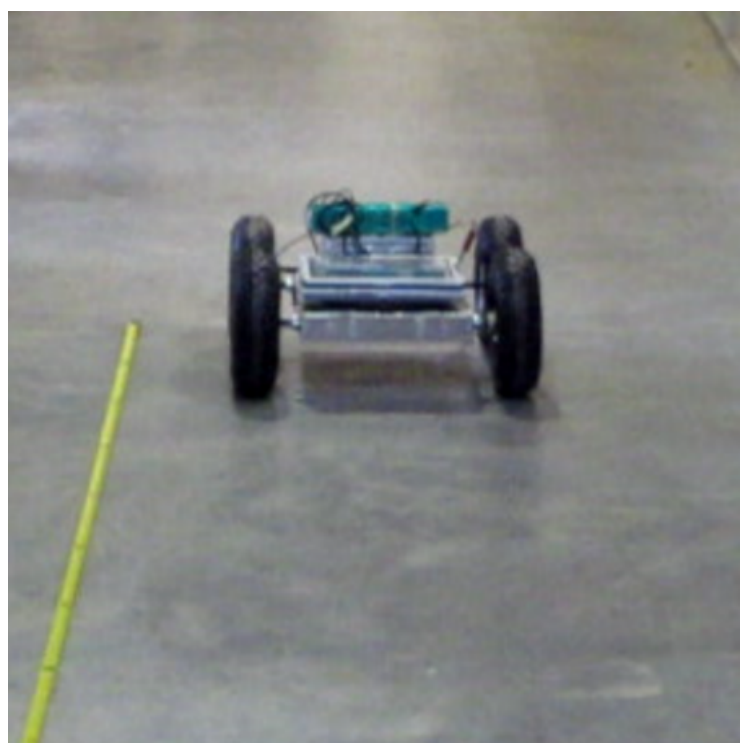

(a)

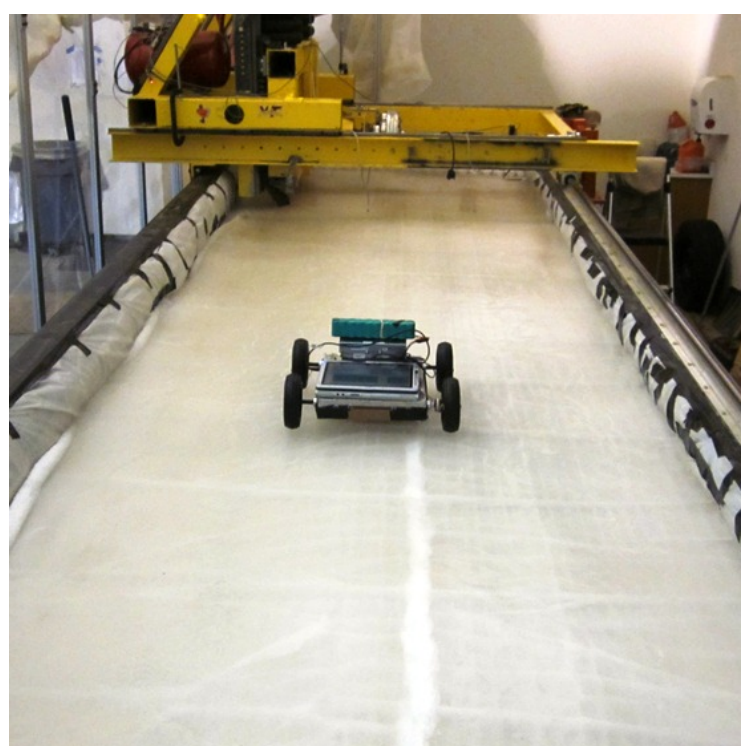

(b)

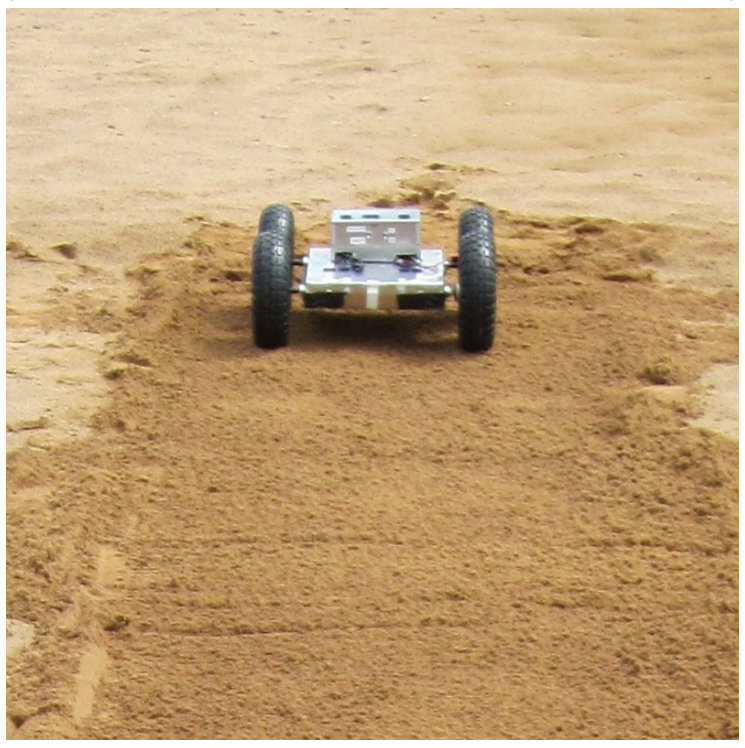

(c)

Figure 4.2: Photograph of robot on test surfaces

(a) polished cement, shown with 10" tires and $10 \mathrm{lb}$ weight, (b) ice, shown with 6" tires, and (c) sand, shown with 10" tires and battery and computer removed (not a testing configuration). 
The maximum sampling rate of $1200 \mathrm{~Hz}$ for the DAQ is reduced by one-half for every additional active channel. Therefore, only two signal conditioning circuits were created and attached to the DAQ for a given test. For each combination of the previously described parameters, two tests were run with Sensors 1 and 2 connected and two tests were run with Sensors 1 and 3 connected. The resulting sample rate was $600 \mathrm{~Hz}$.

\subsection{Surface Preparation and Characterization}

Each of the three surfaces required specific methods of preparation. In this section, the preparation of the two hard test surfaces (polished cement and ice) is described, followed by the measurements taken to characterize the surface.

\subsubsection{Hard Surface Preparation}

A level portion of the indoor polished cement floor was selected that was free from bumps and cracks. The surface was swept and dusted prior to testing.

The ice surface was prepared for another ongoing study in the AVDL. A system of tubes, shown in Figure 4.3, designed for a home ice skating ring cooled the surface. The tubes contain $50 \%$ ethylene glycol and $50 \%$ water. The ice was prepared by a cycle of adding a thin layer of water, allowing it time to freeze, then adding another thin layer. A photograph of the robot on the ice surface, outfitted with the 6" tires, is shown in Figure 4.2b.

\subsubsection{Measurement of Static Coefficient of Friction of Hard Sur- faces}

For the hard surfaces, the static coefficient of friction was measured with a 825A American Slip Meter Horizontal Pull Slipmeter. This instrument uses the testing methodology in ASTM Standard F609-05 to measure the static coefficient of friction [8]. Results are provided in Section 5.1.1.

\subsubsection{Sand Preparation}

A recreational sand volleyball court was used for the tests on sand. The most level portion of the court, determined visually, was selected. The sand was prepared with a 3' wide rake. The rake had teeth 2.5" long, with a width of 0.25 " each and 0.75 " separation between teeth. At the start of testing, sand was displaced to a depth of approximately 3-4", then was smoothed with the rake. A track of sand approximately 4' wide and 15' long was prepared, as can be seen in Figure 4.4a.

All tests for all tires were completed from the same starting area, moving in the same direction. This allowed for any small changes in elevation to be consistent across tests. Each test, the starting position was moved a couple of inches to the side to ensure that the robot would not cross the same section of sand in different tests. If the robot unexpectedly crossed 


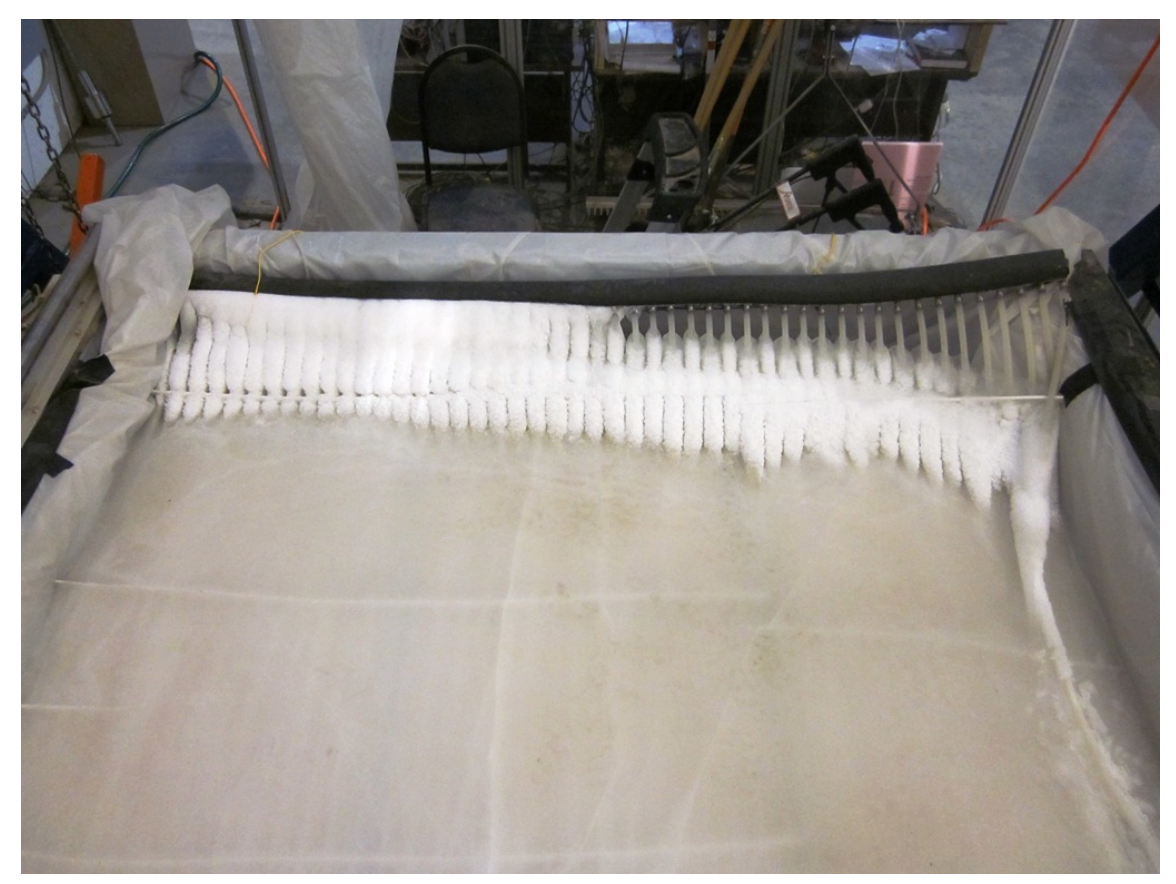

Figure 4.3: Tube system used to create and maintain ice testing surface.

a rut from a previous test, the test data was erased the test was repeated. A series of noncrossing tracks on the prepared sand surface can be seen in Figure 4.4b. When all prepared terrain had been traversed, the testing region was re-raked. Between each test, the tires were wiped with a scrub brush in order to dislodge any sand compacted into the tread.

\subsubsection{Measurement of Cone Index of Sand}

A CP40II Cone Penetrometer was used to measure the cone index of the sand. The cone index is a measurement of the force per unit area of resistance to pushing a required to push a small metal cone into the ground $[6,26,43,71]$. The equation for the cone index CI is

$$
\mathrm{CI}=\frac{F}{A}
$$

where $F$ is the force necessary to push the cone with a face size of $A$ into the terrain $[43,71]$.

Each day of testing, the cone index was taken at 8 locations along the prepared tract of sand. The locations were approximately evenly spaced. The interval on the cone penetrometer was set to $15 \mathrm{~mm}$. The meter averages all of the readings for the cone index over the interval. The cone index measurements are provided in Section 5.1.2.

\subsubsection{Temperature of Tires}

The temperature of the outside surface of the tires was measured and recorded throughout testing using a Craftsman (Hoffman Estates, IL) Model 50466 Non-Contact High Tempera- 


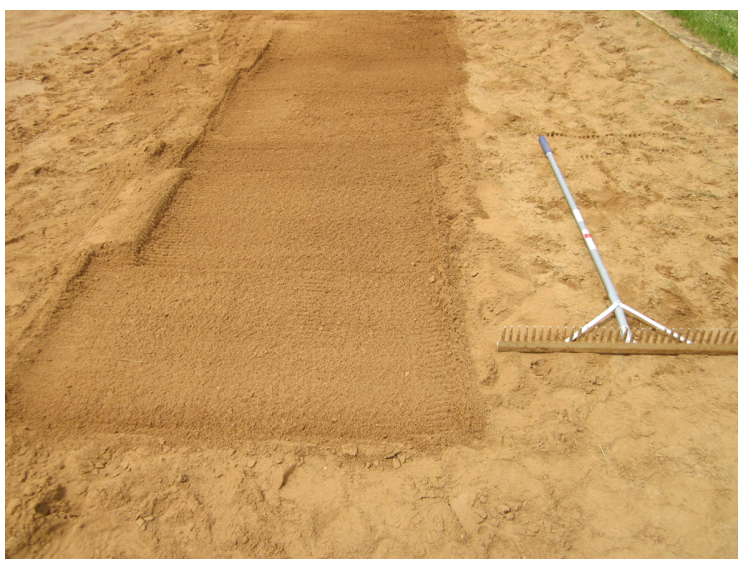

(a)

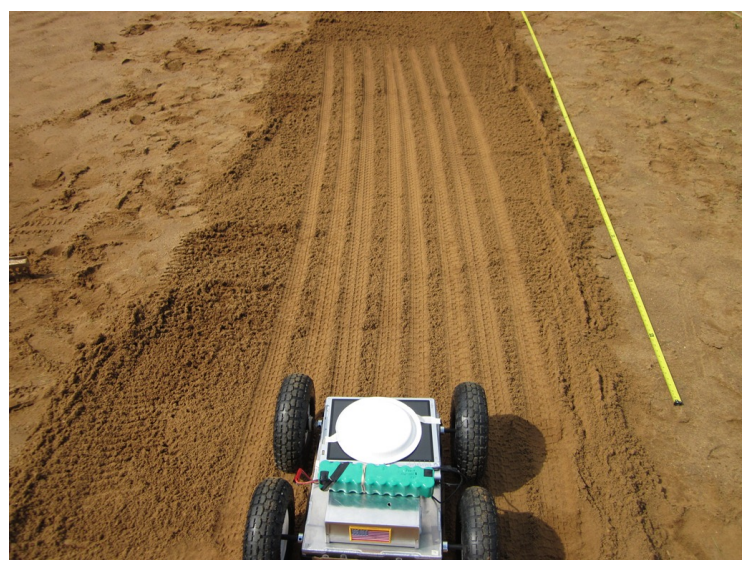

(b)

Figure 4.4: Photographs of prepared sand

(a) before tests with 3' rake shown and (b) after tests with series of non-crossing ruts seen.

ture InfraRed Thermometer. The temperature was recorded for approximately every 2 tests on ice. Because the polished cement and the tire were at room temperature, the temperature was only recorded once at the start of testing. Results are given in Section 5.1.3.

\subsection{Measurements Taken During Each Test}

During the test, the robot and all data collection was controlled by the LabView VI running on the the on-board computer, described in Sections 3.3.4 and 3.3.3. Through the LabView VI, the computer communicated via serial connections with both the motor controller (Section 3.1.4) and the DAQ (Section 3.3.2). The configuration parameters, described Section 4.3.1 pre-set by the operator, were recorded for each test. During the test, measurements were taken for the speed and power levels of the motors and the voltage from the piezoelectric sensors via the DAQ. The parameters and measurements obtained from the motor are described in Section 4.3.2.

\subsubsection{Motor Configuration Parameters From Motor Controller}

The motor controller was connected to the on-board laptop with a USB cable. For each test, the LabView VI sent a command to the motor controller that instructed it to run the preloaded script. The motor controller would then read several of the configuration parameters to the LabView VI. For each motor channel, these parameters were:

- Encoder counts per revolution (PPR)

- High limit for the encoder (PPR)

- Maximum speed (RPM) 
- Maximum acceleration and deceleration $(\mathrm{RPM} / \mathrm{s})$

- Maximum current (A)

- Battery voltage $(10 \mathrm{~V})$

The purpose of recording these parameters was so that the consistency of the robot configuration could be verified between tests.

\subsubsection{Speed and Power Levels From Motor Controller}

Once the configuration parameters were received by the LabView VI from the motor, a command was automatically sent to motor controller to start a particular test sequence. The power sequence, described in Section 4.1.2, would then commence and the DAQ would begin making measurements. During each test, the motor controller read the speed and power level for the right and left sides to the computer at $4 \mathrm{~ms}$ intervals. The speed was measured by the encoders on the front right and front left wheels and given as a relative speed. The relative speed $\omega_{\text {rel }}$ is related to the actual speed $\omega(\mathrm{rad} / \mathrm{s})$ by

$$
\omega_{r e l}=\frac{1000}{60} \frac{\omega}{\omega_{\max }}
$$

where $\omega_{\max }$ is the maximum RPM, configured by the user.

The power was calculated using Equation 4.1 from the current distributed to each side and the battery voltage. The power value provided by the controller was actually the relative power $P_{\text {rel }}$, determined from

$$
P_{\text {rel }}=1000 \frac{P_{\text {mot }}}{P_{\text {max }}}=1000 \frac{V_{\text {batt }} I_{\text {mot }}}{V_{\text {batt }} I_{\text {max }}}
$$

where $P_{m o t}$ is the actual power given to a particular motor, $P_{\max }$ is the maximum theoretical power, based on the battery voltage $V_{\text {batt }}$ and maximum current $I_{\max }$. The motor power is based off of the current distributed to that motor channel $I_{m o t}$. With each speed and power reading, a reading of $t_{m o t}$, the integer number of milliseconds since the start of the test was also recorded.

The average measured power and speed for each test configuration is provided in Section 5.2 .2 .

\subsection{Data Processing}

Once all of the tests were conducted, data analysis was conducted in Matlab (Mathworks, Natick, MA) [33]. The preliminary corrections made for all tests are discussed in Section 4.4.1. Next, each rotation of the wheel was separated. The process for this is given in Section 4.4.2. Finally, the signal from the piezoelectric sensor for each rotation of the wheel was characterized using the methods described in Section 4.4.3. 


\subsubsection{Pre-Processing DAQ Error Correction}

When the differential inputs to the DAQ are short circuited, the output reading should be 0 V. Because of offset error, discussed in Section 3.3.2, the actual reading will be different than $0 \mathrm{~V}$. This error must be accounted for and subtracted out for future tests using that channel. Sensor 1 was always plugged into channel Ai0 of the DAQ, which had an average offset error of $+0.0054 \mathrm{~V}$. Sensors 2 and 3 were always plugged into channel Ai1 which had an average offset error of $-0.0036 \mathrm{~V}$. Corrections for these offset errors were made for all tests.

\section{Gain Correction}

As shown in Table 4.2, the gain of the signal conditioning circuit was different for sensor 1 compared to sensors 2 and 3. Because of the difference in $R_{2}$ in the amplifier circuit (220 vs $100 \mathrm{k} \Omega$ ), the output for all sensor 1 measurements was multiplied by 2.2 .

\section{Encoder Reset Correction}

The robot controller had a set maximum that the encoder counter could reach before it would reset to zero because of the limitations of the number of bits of the counter. When the counter would reset, the speed reading would be extremely high for one reading. To correct for this, a filter was run where if a relative speed measurement varied from the previous measurement by more than 300 , the speed reading would be changed to equal the previous value.

\subsubsection{Separation of Wheel Rotations}

In order to conduct an analysis, each rotation of the wheel needed to be separated. There were several steps in this process that are described here.

\section{Conversion of Data to Evenly Spaced Angles}

The measurements from the DAQ were taken at evenly spaced time intervals. I will refer here to the set of time measurements corresponding to the DAQ measurements as $t_{d a q}$ which are evenly spaced by $\frac{1}{600} \mathrm{~s}$. These times did not necessarily correspond to the approximately evenly spaced time intervals for the measurements of speed and power from the motors, which I will refer to here as $t_{m o t}$. The $t_{m o t}$ times were spaced by approximately $4 \mathrm{~ms}$. This spacing is approximate because there is a small amount of time during which the script runs because the $4 \mathrm{~ms}$ timer is restarted. In order to use all of the recorded measurements together, the data was converted so that all DAQ and motor measurements were approximated at evenly spaced angles. The process for this conversion is as follows:

1. Convert from $\omega_{\text {rel }}$ to $\omega$ using equation 4.11, shown from Figure 4.5a to Figure 4.5b. 
2. Using the MATLAB command cumtrapz, integrate $\omega$ over $t_{m o t}$ to obtain the wheel angle $\theta$, see Figure $4.5 \mathrm{~b}$.

3. Using the MATLAB command interp1, interpolate $\theta$ vs $t_{m o t}$ to $\theta$ vs $t_{d a q}$.

4. Create a matrix of evenly spaced angles ranging from 0 to the maximum angle traveled. For the case shown in Figure 4.5b, this is about 49 rad.

5. Identify the location of the forward and reverse velocity regions and divide data.

6. Use $\theta$ vs $t_{m o t}$ with $\omega$ vs $t_{m o t}$ and $P$ vs $t_{m o t}$ to interpolate $\omega$ and $P$ over the evenly spaced $\theta$ matrix, separately for forward and reverse regions. The result for $\omega$ is shown in Figures $4.5 \mathrm{c}$ and $4.5 \mathrm{~d}$.

7. Use $\theta$ vs $t_{d a q}$ with $V$ vs $t_{d a q}$ to interpolate the DAQ measurements over the evenly spaced $\theta$ matrix, separately for forward and reverse regions.

In the case of the reverse tests, the angle relative to the starting angle would start at a large positive number and decrease to zero. To aide in comparison of data, the measurements for the reverse tests start at zero (corresponding to the beginning of the negative power region) and increase to the maximum $\theta$ (corresponding to the time at the end of the test). This is shown with the symbols in Figure 4.5.

\section{Division of Each Rotation}

The next step was to divide up each rotation of the wheel. This was done by examining the sensor 1 signal. This method allows for the complete rotation to occur within the constant velocity region.

1. Identify from the speed $\omega$, shown in Figure 4.6 a the angular starting position of the constant velocity region (forward or reverse), shown with the red dotted line labeled $\mathbf{i}$ in Figure $4.6 \mathrm{~b}$

2. Find the location of the minimum value of the sensor 1 reading that occurs within 1 rotation $(2 \pi \mathrm{rad})$ ahead of the start of the constant velocity region. The minimum is labeled with iii in Figure 4.6b, which falls between the lines labeled $\mathbf{i}$ and ii.

3. Step $1 / 2$ rotation ahead of this point. This will be the location of the start of the first rotation, labeled iv in Figure 4.6b.

4. Divide up the segments for every rotation until reaching the end of the constant velocity region, shown by the black dash-dotted lines in Figure 4.6b.

5. Repeat for the reverse test.

Once the rotations were separated for sensor 1 , the rotations had to be separated for whichever other sensor (2 or 3 ) was used for that test. The locations of the divisions were taken from the divisions for sensor 1, shifted by the known about of angle between the location of the sensors on the tires. These angles are given in Section 5.3.1. 


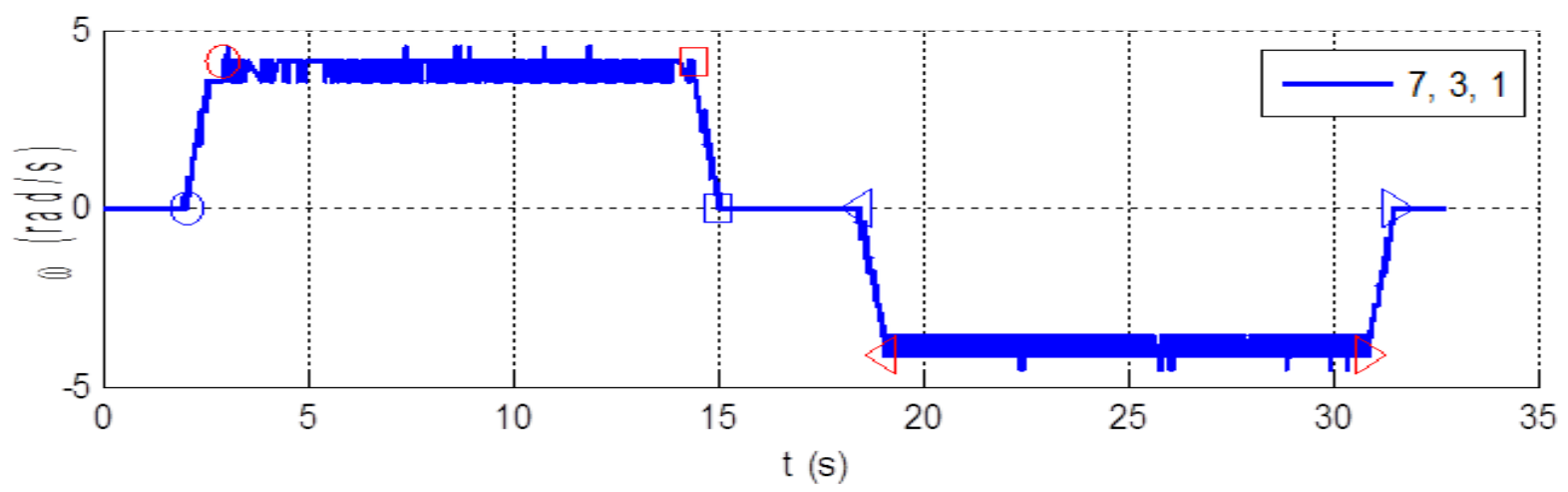

(a)

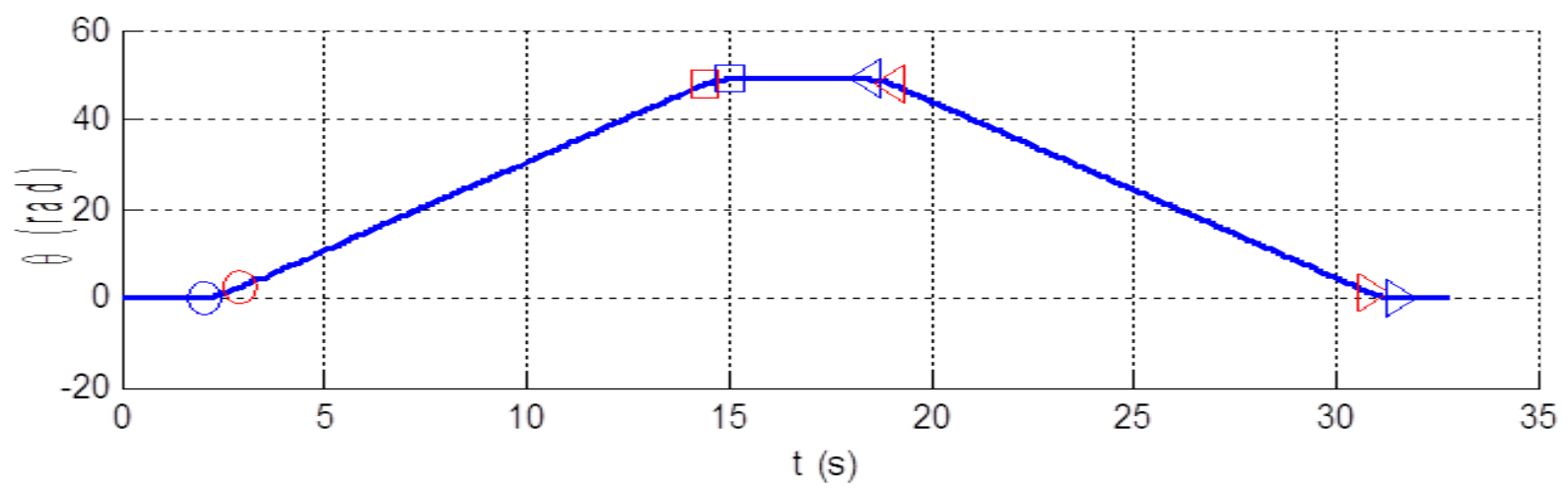

(b)

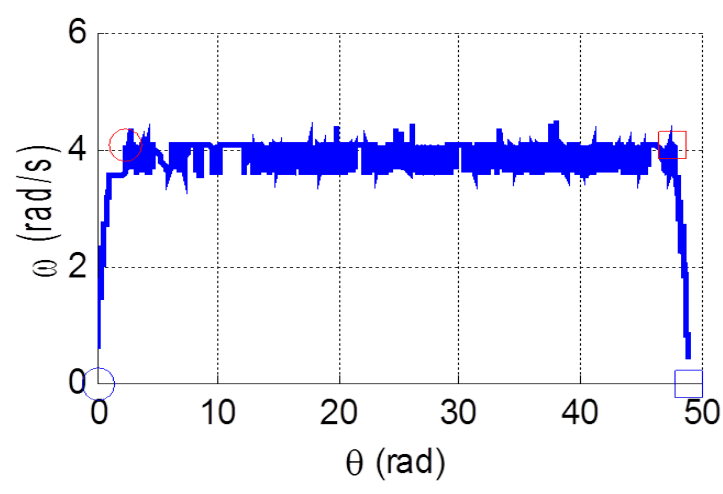

(c)

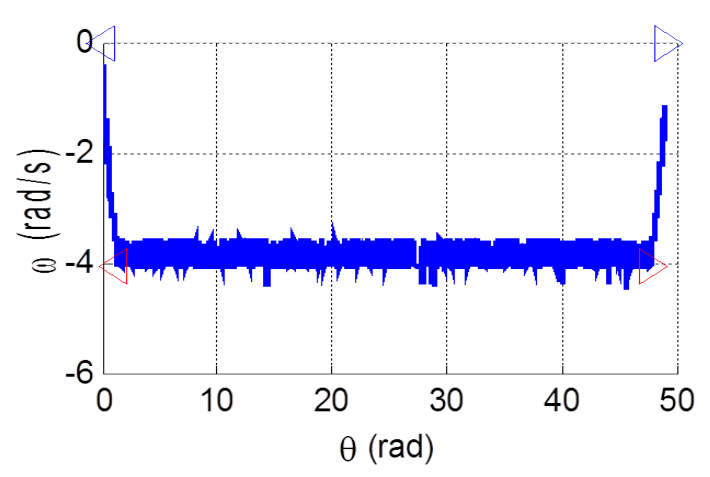

(d)

Note: This example shows the 6" tires with no added load on $50 \%$ applied power on cement.

Figure 4.5: Graphs showing an example of conversion from speed vs time to speed vs angle. (a) speed vs time, (b) angle vs time, (c) speed vs angle (forward only), and d) speed vs angle (reverse only) 


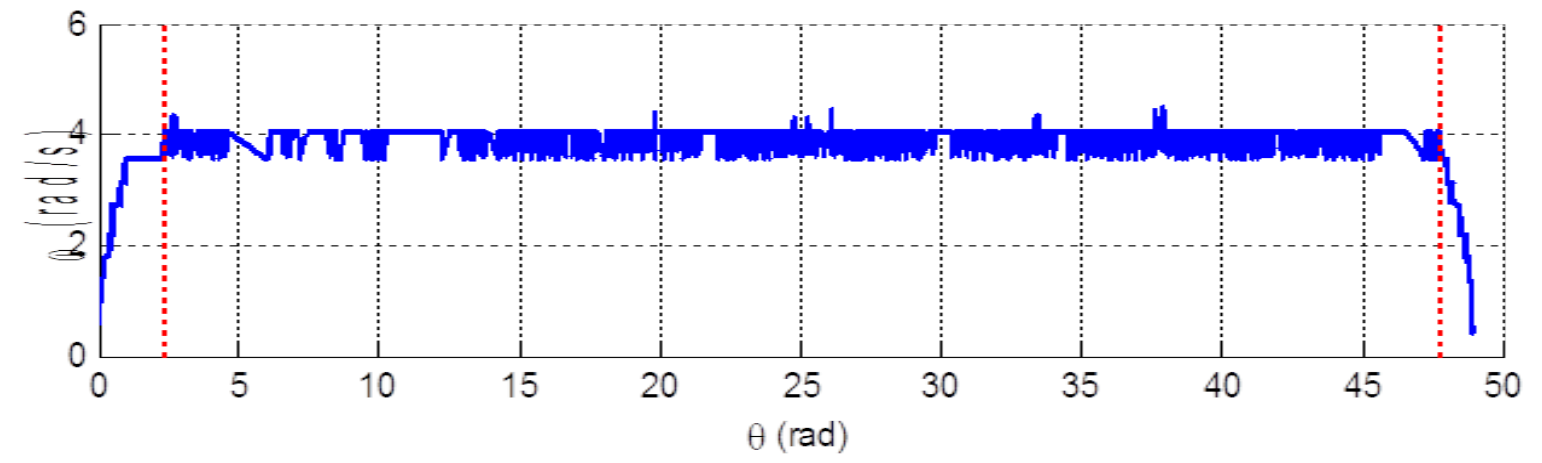

(a)

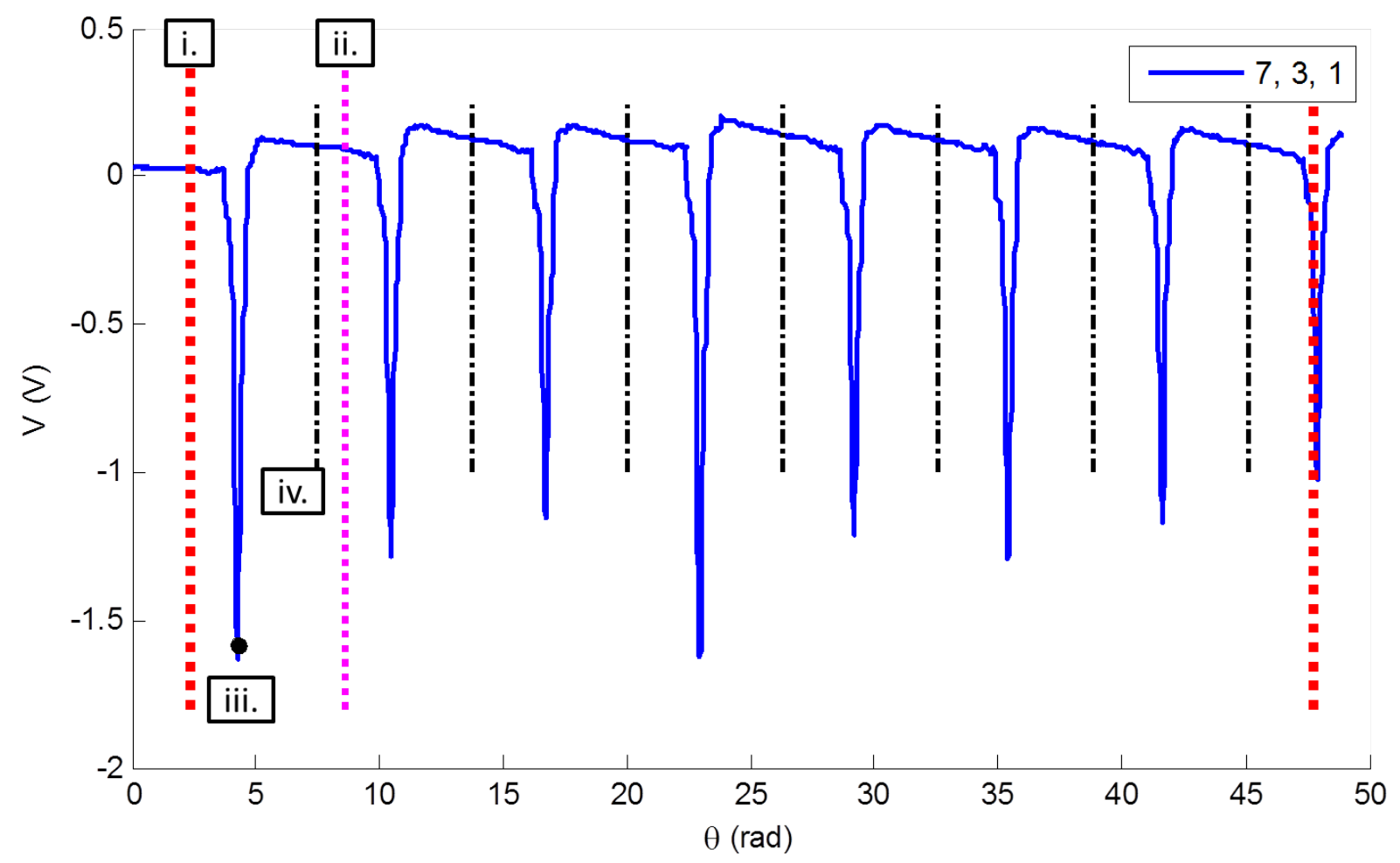

(b)

Note: This example shows the 6" tires with no added load at $50 \%$ applied power on cement. Lowercase roman numerals are described in the test.

Figure 4.6: Graphs showing an example of separation of angle rotations.

(a) speed vs time and (b) angle vs time 


\subsubsection{Signal Characterization}

In order to characterize the signal from each rotation of the wheel, two general parameters were of interest: the amplitude and the width. Selecting a single, simple description of the amplitude and width that would work for all signal shapes was not easy. In this section, I will show some atypical examples that introduced the need for further pre-processing. After this processing, the amplitude and width could be determined.

\section{Measurement of Signal Amplitude}

Measuring the amplitude of the signal could be taken to mean either the maximum range of the signal or maximum absolute value of the signal. Figure $4.7 \mathrm{a}$ demonstrates a case where the sensor had a signal before the start of the test that was still present due to the time constant of the circuit. This caused each individual rotation to appear on a slope. Neither the range nor the maximum absolute value of the signal for any rotation would really capture the characteristic of interest. Therefore, a correction process was introduced. This correction process is also important for the method later introduced to characterize the signal width.

The first step was to correct for the overall slant. The sensor measurements for each rotation were corrected so that the first and last value were the same. This was done with a linear correction. In reality, the offset is exponential by time, but this may require converting all of the measurements back to time intervals in order to correct. I believe the linear approximation will suffice and it is easier to implement. The result is the appearance the the curve has been rotated. This is demonstrated in Figures $4.7 \mathrm{~b}$ and $4.7 \mathrm{c}$ by comparing the lines labeled 'original' and 'rotated'.

The results for the average amplitude of each test condition and a discussion is presented in Section 5.3.3.

The next step is to correct for the offset. In this step, the rotated curve is shifted downward so that the maximum value occurs at $0 \mathrm{~V}$. This is demonstrated in Figures $4.7 \mathrm{~b}$ and $4.7 \mathrm{c}$ by comparing the lines labeled 'rotated' and 'rotated, shifted'.

\section{Measurement of Signal Width}

Several methods were considered for evaluating the width of the signal in a simple, straightforward manner. My original thought was to use the region spanned by the maximum and minimum of the first derivative. Problems with this method arose because many of the signals had a small double peak phenomena. This can be seen in Figure $4.7 \mathrm{c}$, where the most positive slope probably occurs just near the first small extrema, rather than encompassing the entire signal.

Alternatively, a method called the Full Width at Half-Maximum (FWHM) was considered. This method to characterize a signal is used in many fields, for example in image processing to determine the boundaries between and sizes of objects. Figure 4.8 contains a demonstration this technique. The maximum $V$ value in the segment is located. From there, the nearest $\theta$ values in the positive and negative directions that have voltage measurements 


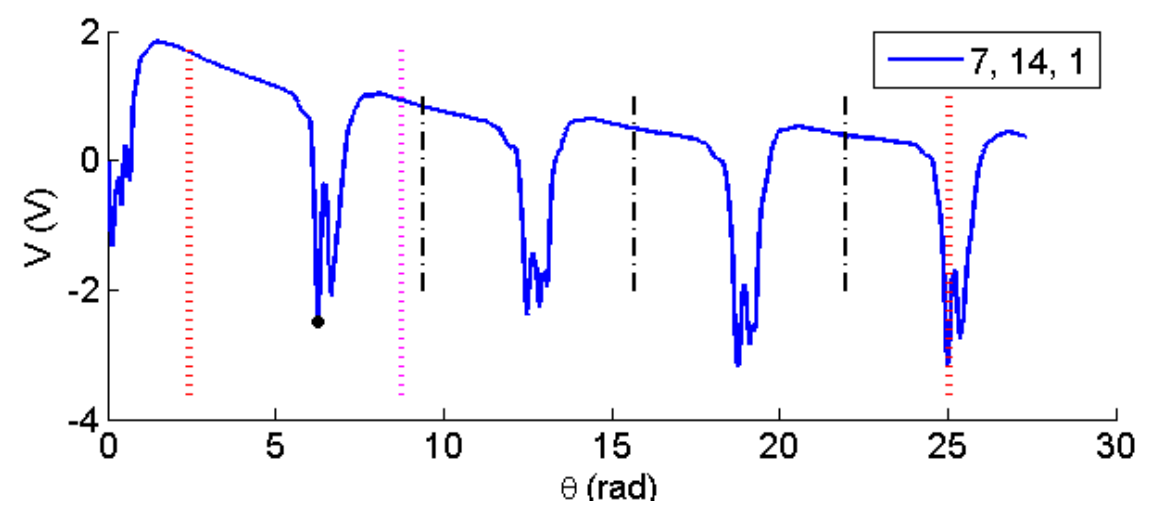

(a)

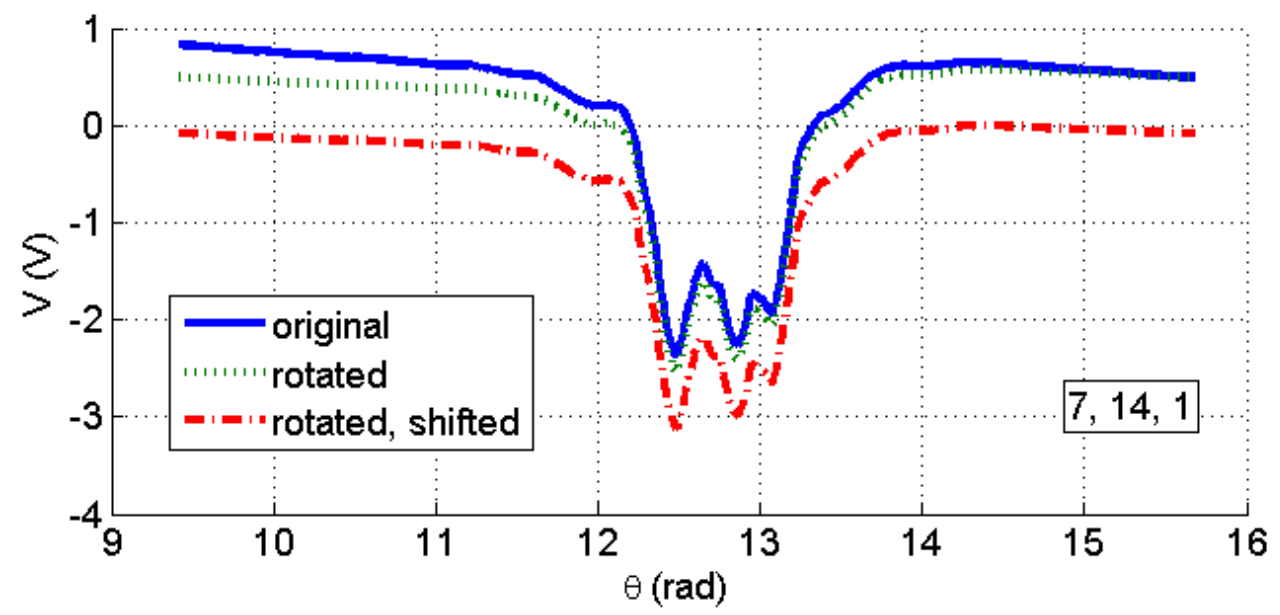

(b)

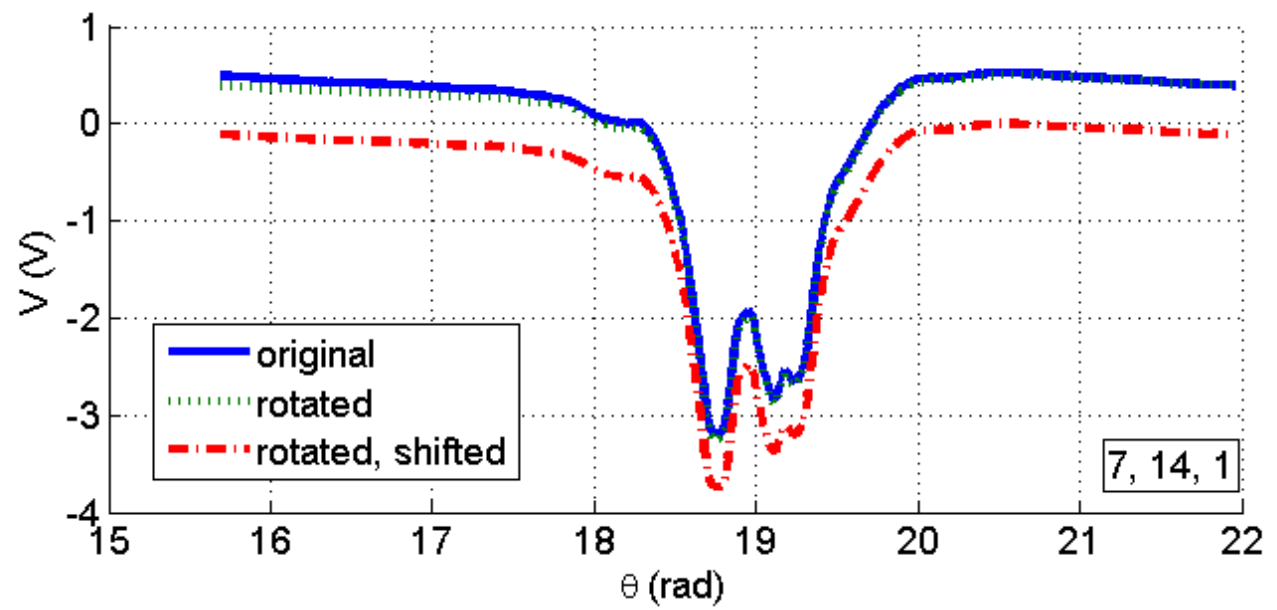

(c)

Note: This example is from the 10" tires with $10 \mathrm{lb}$ payload at $75 \%$ power on cement.

Figure 4.7: Graphs illustrating the process of shifting the signal measured during each rotation of the wheel so that any slants and offsets are corrected.

(a) Original full length signal, (b) Shifting of rotation 1 and (c) Shifting of rotation 2 


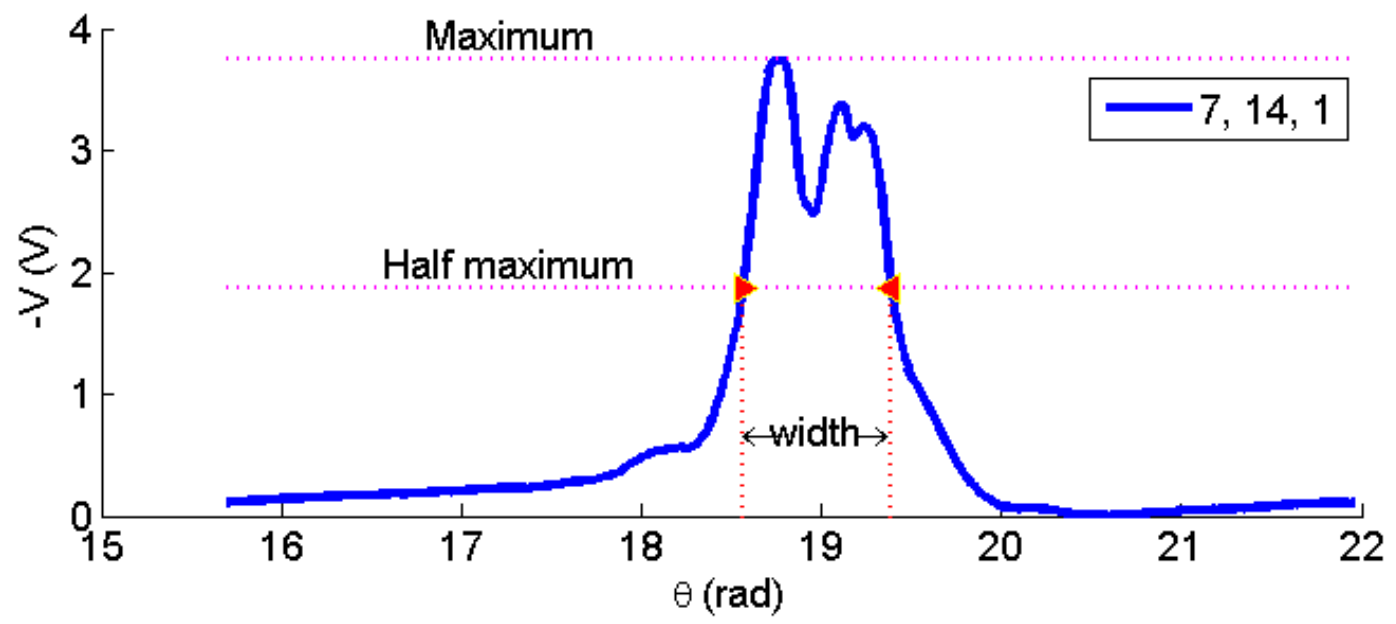

Note: This is the same segment of signal shown in Figure 4.7c, but the y axis has been reversed to be consistent with the name 'maximum' of the technique.

Figure 4.8: Graph demonstrating the full width at half maximum method to characterize the width of the signal for each rotation.

at half of the maximum value. The difference between these $\theta$ values is then the width of the segment.

In practice, some problems arose with the FWHM technique. These problems, the modifications considered for the method, and the results are discussed in Section 5.3.4. 


\section{Chapter 5}

\section{Results and Discussion}

This chapter contains a presentation and discussion of the results from the test series described in Chapter 4. The tests conducted to characterize the surface are presented in Section 5.1. In Section 5.2, results for the load, speed, and power measured for the robot are given. A summary and discussion of the measurements from the piezoelectric sensors is provided in Section 5.3. Finally, the limitations to the present study are discussed in Section 5.4.

\subsection{Surface Characterization}

This section contains the results for characterizing the surfaces. For the hard surfaces (ice and cement), the static coefficient of friction was measured. For sand, the cone index was measured.

\subsubsection{Coefficient of Static Friction for Hard Surfaces}

Measurements were taken for the coefficient of friction on the ice and the polished cement floor in several different directions by the same operator. On the polished cement floor, the coefficient of static friction was found to be $0.72-0.75$. This is less than the value given in Wong [71] for dry cement, which is 0.8-0.9. Within the category of cement and asphalt, there is a range of macro and microtextures. Polished cement is the smoothest of these, which may explain the lower measured coefficient of static friction.

The measured coefficient of static friction on the ice was in the range of 0.15 to 0.18 . This is slightly more than the value of 0.1 provided in Wong [71].

\subsubsection{Cone Index of Sand}

The cone index was measured on both days of testing. Unfortunately, due to technical problems, the data from the first day was erased. On the second day, testing was conducted with the 6" tires. The cone index of the sand was measured on the prepared sand before the

first test was conducted. Following completion of the last test, the cone index was measured 


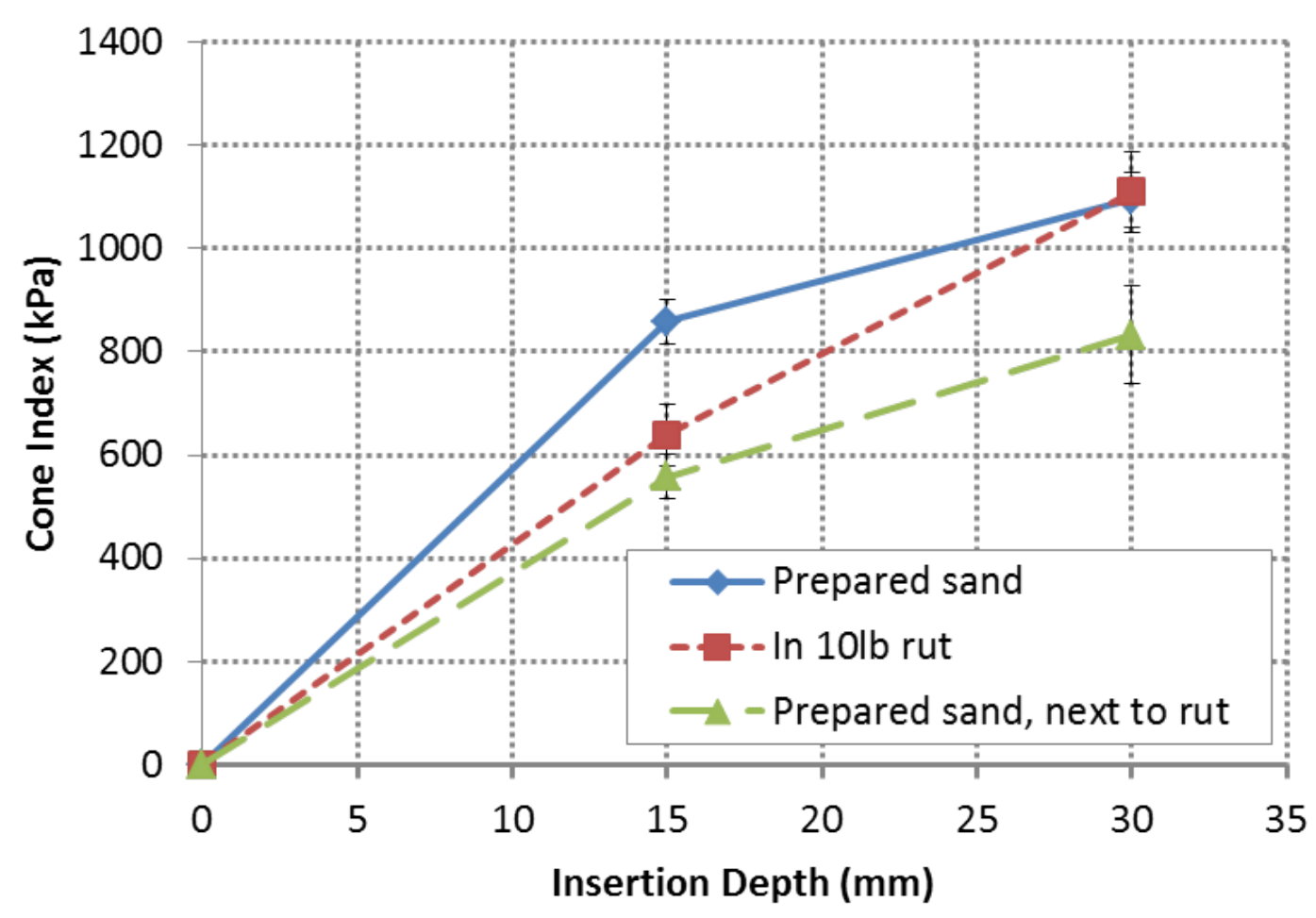

Note: Error bars indicate the standard error.

Figure 5.1: Cone index measurements for sand on the second day (6" tires) of testing.

again. For the final tests, a set of cone index measurements was taken inside one of the ruts created by the 6" tires with $10 \mathrm{lb}$ load. A set of measurements was then taken in the prepared but untraversed sand in positions next to the measurements in the rut. The results for these measurements are shown in Figure 5.1. When the cone penetrometer senses that the insertion speed is lower than a specified threshold, the run is aborted. This occurs if the ground becomes too hard to push through. Typically, the runs were aborted around $35 \mathrm{~mm}$ insertion depth.

The measurement for the cone index on the prepared sand was different at the end of testing compared to the beginning of testing. The cone index was greater within the rut than alongside of the rut, which is as would be expected.

\subsubsection{Temperature}

The robot and tires were stored at approximately room temperature $\left(68-72^{\circ} \mathrm{F}\right)$ prior to testing. The temperature of the tire could change throughout testing due to environmental factors such as air temperature, ground temperature, and sunlight. Sliding friction at the tire-ground interface can generate heat at the tire surface. Testing for the 6" tires and the 10" tires was completed on different days for each of the three surfaces. The range of 
Table 5.1: Range of temperature measurements for top each tire size

\begin{tabular}{ccc}
\hline & $\begin{array}{c}\text { Tire } \\
\text { Temperature }\left({ }^{\circ} \mathrm{F}\right)\end{array}$ & $\begin{array}{c}\text { Ground } \\
\text { Temperature }\left({ }^{\circ} \mathrm{F}\right)\end{array}$ \\
\hline Cement & & \\
6" Tires & 72 & $\times$ \\
10" Tires & 72 & \\
Ice & & $14-20$ \\
6" Tires & $52-65$ & $18.5-19.4$ \\
10" Tires & $50-70$ & \\
Sand To- Tires & $90-114$ & $80-100$ \\
6" To- Tires & $70-80$ & $55-67$ \\
\hline
\end{tabular}

Notes: Measurements taken between tests on the outside surface of top of the tire with a Craftsman 50466 InfraRed Thermometer. No measurements were taken for the polished cement floor.

temperature values measured for the tire surface and the ground surface for each tire size and surface type combination are presented in Table 5.1.

In Section 2.2.4, the temperature sensitivity of PVDF piezoelectric sensors was discussed. Three types of temperature effects should be considered: (1) permanent decay of the piezoelectric strain constant as a result of storage at elevated temperatures, (2) reversible increases or decreases of the piezoelectric strain constant as a result of increases or decreases in temperature, and (3) charge generation as a result of temperature change (pyroelectricity). Because the sensors were stored at or near room temperature between tests, it is not expected that there was long term permanent decay of the piezoelectric strain constants. The temperature effects for each set of tests is discussed here.

The measured temperature was the outside surface of the tire. The temperature of the piezoelectric sensor mounted to the inside surface of the tire is not known. The heat transfer through the tire depend on the thermal properties and thickness of the tire material. The temperature of the piezoelectric sensors was likely closer to the room temperature than the measured value for the outside surface of the tire.

On ice, it was found that the surface temperature of the top of the tire decreased by about $2^{\circ} \mathrm{F}$ during each test. The ice temperature increased throughout each set of tests (a set refers to all of the tests with a certain tire size and surface) which may have been due to increases in the temperature of the air in the room. The robot was removed from the ice half way through each set of tests in order to allow the temperature of the tires to rise. This helped to reduce the range of temperatures at which the tires were tested. It is assumed that the temperature changes of the tire were due primarily to the cool ice surface. Therefore, the portion of the tire that was touching the ice surface between tests was probably cooler than the top of the tire where the measurements were taken. 
It is not known if the cooler temperature of the ice caused a change in temperature of the sensor during each rotation through the contact patch. If it did, then any measured signal for a sensor on the tread may be due to this temperature change. The cooler temperature of the tire on ice compared to on other surfaces may have caused reduction of the piezoelectric strain constants and therefore a reduced signal.

The polished cement surface was indoors in a climate controlled area. The temperature of the tires was only measured once during testing and was found to be $72^{\circ} \mathrm{F}$. It is therefore not expected that temperature had an effect on the observed signal.

Testing of the 10" tires on sand was completed on an cloudy afternoon with an air temperature around $56^{\circ} \mathrm{F}$. The temperature of the tires and the sand surface decreased throughout testing as the air temperature cooled into the evening. The 6" tires on sand were tested mid-day on a sunny day with an air temperature around $70^{\circ} \mathrm{F}$. The temperature of both the sand and the tire increased throughout testing as the sun heated the surfaces. If the sun was primarily heating the top surface of the tire, the bottom surfaces may have been cooler.

Similar to the ice, it is not known how the heat from the sand and the sun may have been transfered to the sensor during each rotation. Any temperature changes that did occur during a rotation would have caused a signal to develop. Throughout test on the sand, elevated temperatures may have caused an increase in the piezoelectric strain constant which would cause stronger signals to be produced as a result of mechanical strain.

\subsection{Measurements of the Robot}

Several characteristics of the robot were known and recorded for each test. In this section, those measurements from the robot are presented, which include the speed, power, and load on each tire.

\subsubsection{Static Load on Each Tire}

For each of the four payload-tire test configurations, the static load was approximated using a set of measurements described previously. The results from these measurements are given in Table 5.2. From these results, we see that the 10" tires were heavier than the 6" tires, which would be expected.

\subsubsection{Power and Wheel Speed}

Approximately every $4 \mathrm{~ms}$ during the tests, the speed and power of the motor was read from the motor controller to the LabView VI. While the motor may have been commanded to go at a certain power level, because the system was operating in an open loop, the result was that it did not always go at the specified level. In Tables 5.3 and 5.4, the average values for speed and power are provided for the 6" and 10" tires, respectively. Because the speed and power readings for the reverse velocity region were negative, these averages represent 
Table 5.2: Weight distribution across four wheels of the robot, based on tire and payload configuration.

\begin{tabular}{lccccc}
\hline & \multicolumn{2}{c}{ 6" Tires } & & \multicolumn{2}{c}{$10 "$ Tires } \\
\cline { 2 - 3 } \cline { 5 - 6 } & Payload $=0 \mathrm{lb}$ & Payload $=10 \mathrm{lb}$ & & Payload $=0 \mathrm{lb}$ & Payload $=10 \mathrm{lb}$ \\
\hline$W_{F L}(\mathrm{~kg})$ & 3.34 & 5.04 & & 4.11 & 5.82 \\
$W_{F R}(\mathrm{~kg})$ & 3.35 & 5.08 & & 4.13 & 5.86 \\
$W_{R L}(\mathrm{~kg})$ & 3.45 & 4.02 & & 4.22 & 4.78 \\
$W_{R R}(\mathrm{~kg})$ & 3.46 & 4.04 & & 4.24 & 4.82 \\
\cline { 2 - 3 }$W_{\text {total }}(\mathrm{kg})$ & 13.60 & 18.18 & 16.70 & 21.28 \\
\hline
\end{tabular}

Note: Loads for the 10" tires with $10 \mathrm{lb}$ of payload were calculated from the other three configurations, not measured.

Table 5.3: Speed and power for 6" tires

\begin{tabular}{lcccccccc}
\hline & \multicolumn{3}{c}{ Wheel Speed $(\mathrm{rad} / \mathrm{s})$} & & \multicolumn{3}{c}{$\% P_{\max } \times 10$} \\
\cline { 8 - 9 } & $\mathrm{P}=50$ & $\mathrm{P}=75$ & $\mathrm{P}=100$ & $\mathrm{P}=50$ & $\mathrm{P}=75$ & $\mathrm{P}=100$ \\
\hline Payload =0 lb & & & & & & & \\
$\quad$ Ice & 3.97 & 7.02 & 10.62 & & 374.9 & 655.5 & 998.6 \\
$\quad$ Cement & 3.93 & 6.95 & 10.51 & & 374.9 & 655.4 & 999.2 \\
$\quad$ Sand & 4.01 & 7.16 & 10.84 & & 374.9 & 655.9 & 999.0 \\
Payload =10 lb & & & & & & & \\
$\quad$ Ice & 3.89 & 6.93 & 10.52 & & 374.9 & 655.2 & 998.4 \\
$\quad$ Cement & 3.94 & 6.96 & 10.54 & & 374.9 & 655.5 & 999.4 \\
$\quad$ Sand & 3.88 & 7.00 & 10.66 & & 374.9 & 655.8 & 999.4 \\
\hline
\end{tabular}

the average of the absolute value. The average was only taken for values occurring in the constant velocity regions.

\section{Differences in Power}

For the applied power level of $50 \%$, the result was actually that approximately $37.5 \%$ of $P_{\max }$ was delivered in all cases. For the applied power level of $75 \%$, the actual delivered power was approximately $65.5 \%-65.6 \%$ of $P_{\max }$. The case where the applied power was $100 \%$, the actual power delivered was very close to that, approximately $99.8 \%-99.9 \%$ of $P_{\max }$. It is important to note that $P_{\max }$ may have varied throughout a set of tests and between tests as the power level of the battery changed. 
Table 5.4: Speed and power for 10" tires

\begin{tabular}{lcccccccc}
\hline & \multicolumn{3}{c}{ Wheel Speed $(\mathrm{rad} / \mathrm{s})$} & & \multicolumn{3}{c}{ Power $\left(\% P_{\text {max }} \times 10\right)$} \\
& $P=50$ & $P=75$ & $P=100$ & & $P=50$ & $P=75$ & $P=100$ \\
\hline Payload =0 lb & & & & & & & \\
$\quad$ Ice & 4.05 & 7.14 & 10.80 & & 374.8 & 655.6 & 998.9 \\
$\quad$ Cement & 3.94 & 6.99 & 10.60 & & 374.8 & 655.5 & 998.0 \\
$\quad$ Sand & 3.74 & 6.74 & 10.15 & & 374.7 & 655.7 & 998.2 \\
Payload = 10 lb & & & & & & & \\
$\quad$ Ice & 3.98 & 7.08 & 10.63 & & 374.8 & 655.6 & 998.3 \\
$\quad$ Cement & 3.87 & 6.90 & 10.45 & & 374.9 & 655.2 & 998.7 \\
$\quad$ Sand & 3.55 & 6.52 & 9.85 & & 374.9 & 655.4 & 998.2 \\
\hline
\end{tabular}

\section{Effect of Applied Power on Wheel Speed}

For both tire sizes and all test configurations, higher wheel speeds were recorded for higher power levels. This is consistent with expectations.

There was more variance seen between payload, surface, and tire size combinations for the average wheel speed compared to the variance seen between tests for the average power.

For two wheels with the same level of applied power, differences in wheel speed can suggest differences in motion resistance or differences in the level of slip at the tire-ground interface. In general, lower speeds could indicate greater motion resistance or less slip. Higher speeds could indicate lower motion resistance or more slip. This can be seen in Equations 2.1 and 2.2 .

\section{Effect of Payload on Wheel Speed}

Greater payload will increase the rolling resistance and therefore decrease the wheel speed. By Equation 2.3, increases in payload will result in increases in $F_{x p e a k}$. This may keep slip levels low for a given power level. Both greater rolling resistance and lower slip should result in a lower velocity.

For all test conditions on sand and ice, the wheel velocity was lower with the 10 lb payload, as was expected. On cement, the addition of the $10 \mathrm{lb}$ payload resulted in lower speeds for the 10" tires (as expected) but slightly higher speeds with the 6" tires. This suggests that any changes in rolling resistance or slip for the 6 " tires that should have resulted in decreased wheel speed were not significant.

Because the 6" tires on ice did not appear to have experienced a significant change in rolling resistance with the addition of a $10 \mathrm{lb}$ weight, it is reasonable to assume that the tires would not experience a change in rolling resistance on ice. Therefore, the decrease in wheel speed on ice experienced by the 6" tires with the addition of a $10 \mathrm{lb}$ weight may be due to a decrease in slip. For the 10" tires, the decrease in wheel speed could be due to an increase in rolling resistance or a decrease in slip. 


\section{Effect of Tire Type on Speed}

The tread pattern of the 10" tires suggests that these tires were designed to offer traction on off-road surfaces. It was therefore expected that the 10" tires would experience less slip and lower speeds on sand compared to the 6" tires. The results were consistent with this expectation.

The relative grip of the tires on hard surfaces and the level rolling resistance is not known. The results show that the wheel speed on ice was faster for the 10" tires compared to the 6 " tires. On cement, the wheel speed was faster for the 10" tires compared to the 6 " tires only in the cases where there was no added payload. When the $10 \mathrm{lb}$ payload was added, the 10" tires were slower than the 6" tires.

\section{Effect of Surface Type on Wheel Speed}

For the 6" tires with no added payload and the 10" tires with and without added payload, the wheel speed was higher on ice than on cement. Due to the lower coefficient of friction of the ice compared to the cement, it is believed that the tires reached higher levels of slip at the same power level. With the $10 \mathrm{lb}$ payload added, the wheel speed for the 6" tires became lower on the ice compared to the cement. The reason for this is not known.

It was expected that the wheel would experience greater rolling resistance on sand compared to ice or cement which would lead to lower wheel speeds. On the other hand, the tire may not achieve a high level of grip with the sand, resulting in increased slip and higher wheel speed. The speed measurements may indicate whether the rolling resistance or the slip had a greater effect on wheel speed for each case. For the 10" tires, lower speeds were recorded on sand compared to cement for all cases. This suggests that the tires had good grip with the sand (low slip) and/or experienced high rolling resistance. For the 6" tires, the speed was higher on the sand than the cement for most cases. This suggests that the 6" tires had higher slip on sand than on cement and may have experienced less rolling resistance than the 10" tires. The exception to this was the test with the 6" tires and $10 \mathrm{lb}$ payload at $50 \%$ applied power. This was the only test with the 6" tires were the wheel speed was lower on sand than on cement. In this case, the wheel may have gotten better traction thanks to the higher load and experienced greater rolling resistance.

\section{Summary of Speed and Power Results}

The resulting power output of the motor was typically less than the applied level, but the resulting power was generally consistent across test conditions. In future studies, a closed loop power mode could be used to offer greater control over the power level.

The measurements of wheel speed suggest that the 10" tires had high slip on the ice and low slip on the sand. The results also suggest that the 10" tires experienced greater rolling resistance on sand than cement. The results from the 6" tires are more varied. Without added payload, the results suggest that the 6" tires had higher slip on the ice than the cement. With the added payload, the wheel speed was lower on ice than on cement. With 
the exception of the $10 \mathrm{lb}$ case at $50 \%$ applied power, the wheel speed was faster on sand than on cement, suggesting a higher level of slip and lower grip.

\subsection{Results from Piezoelectric Sensors}

The purpose of the tests was to determine what information could be extracted from the piezoelectric sensor measurements. In this section, the measurements taken from these sensors are presented along with a discussion of the results.

\subsubsection{Sensor Placement}

It turned out to be challenging to place the sensors in a specific location on the inside of the tire. Despite being cleaned with rubbing alcohol, the tire interior has very low surface energy, making it difficult to bond at an adhesive. After positioning the sensors in a desired location, the process of inserting and inflating the tubes would sometimes move the sensors out of place. The result was that the sensors were not always aligned straight with the longitudinal axis and the placement of the sensors relative to one another was not consistent between the 6" and 10" tires. Efforts were made to check the sensor placement by tapping on the tires and monitoring the signal output, but this method could not detect very small changes in location.

The precise location of each sensor was not known until after testing was completed and the tires could be deflated and tubes removed. After several weeks of being pressed into the tire, the double sided tape had adhered to the wall and the position of the sensor was able to be identified. A paint pen was used to mark the location of the sensors on the outside of the tire. From the markings, the location of the sensors relative to one another was determined, as well as the orientation of sensors 1 and 3 relative to the longitudinal axis. These angles are provided in Table 5.5. The angles were determined by taking photographs of the paint pen markings and then using the image analysis software Image J (NIH, Bethesda, MD) [53] to measure the angles.

Table 5.5: Angular differences in location between the piezoelectric sensors.

\begin{tabular}{ccc}
\hline & 6" tires & 10" tires \\
\hline$\theta_{12}(\mathrm{rad})$ & 0.08 & 1.44 \\
$\theta_{13}(\mathrm{rad})$ & 1.01 & 2.46
\end{tabular}

Note: $\theta_{i j}$ is the angle by which sensor i leads sensor $\mathrm{j}$ while the robot is moving in the forward direction. For a diagram of these angles, see Figure 3.7. 


\subsubsection{Qualitative Analysis of Measurements from Piezoelectric Sen- sors}

A representative signal from each sensor for a single rotation of the tire is shown in Figure 5.2. An image of the location of the sensor along the tread pattern is shown in the inset of Figures 5.2a, 5.2b, 5.2e, and 5.2f.

The signals collected from sensor 2 on the sidewall of the 10" tires were very weak for all test conditions. This can be seen in Figure 5.2d. It is not known if this was because the signal was in fact weak or if there was a bad connection between the sensor and the signal conditioner. It is possible that a strong signal was not picked up because of the small size of the sensor relative to the length of the sidewall. Whatever the reason may be, no analysis was conducted on sensor 2 for the 10" tires. The size and shape of the weak signal made it difficult to use the previously established methods to determine the amplitude and the width.

Some qualitative observations can be made from viewing these curves. For the 6" tires, the narrowest signal is from sensor 2 (Figure 5.2a), which is mounted on the sidewall. This makes sense because orientation of the sensor means that it spends the least amount of rotation aligned with any portion of the contact patch. Because of the weak signals for sensor 2 for the 10" tires, similar observations cannot be made.

For sensors 1 and 3, positioned along the tread, characteristics of the tread pattern are visible in the output signal. If the portion of the outside of the tread that has the tread pattern (in the grey areas of the inset diagrams) is more rigid and is in contact with the ground, we would expect less bending, greater normal pressure, and greater shear forces to occur as those areas pass through the contact patch. The result is that the strain of the sensors is not smooth throughout the contact patch. Figure 5.2e shows three small bumps near the center of the signal. The tread pattern shown in the inset shows three repetitions of the 6" tread pattern along the length of the sensor. Likewise, Figure 5.2f shows two larger bumps at the bottom of the signal. The tread in the inset shows two repetitions of the 10" tread pattern. The size of the local bumps is larger for the 10" tires than for the 6" tires. This may be because the tread pattern for the 10" tires is more defined.

It should be noted that throughout other tests, the number of bumps at the bottom of the curve was consistent, however the relative magnitudes varied. The double (or sometimes triple) bump phenomena, like that seen clearly in Figure 5.2b was not as pronounced for tests on sand. An explanation for this is that the sand caused the load to be distributed even in the recessed areas of the tread, therefore reducing variations in pressure and deflection throughout the length of the contact patch.

We can see that the measurements are only in one direction and do not go dramatically down and up from the original level. This is different than the results found by Yi and Liang [73]. The reason for this lies in the signal conditioner. While the signal conditioner is not explicitly described in the aforementioned paper, it can be inferred that the purpose of the conditioner was not to increase the time constant. Therefore, Yi and Liang [73] was measuring changes in strain, rather than strain directly. For our study, the signal conditioner worked to lengthen the time constant, allowing for a static signal to be recorded for short 


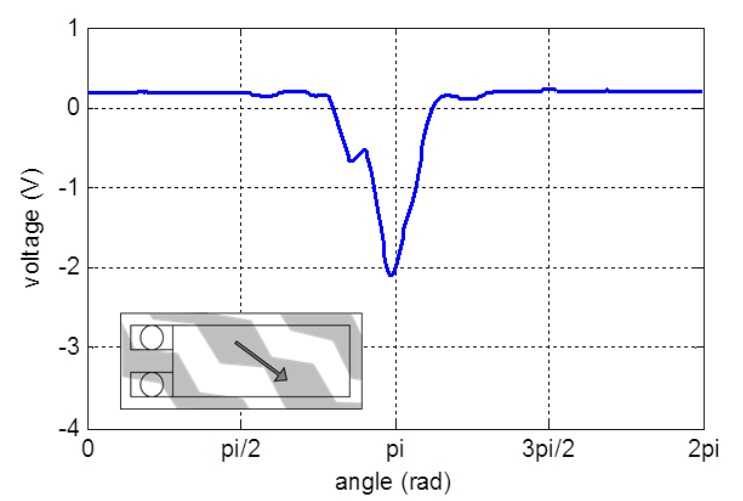

(a)

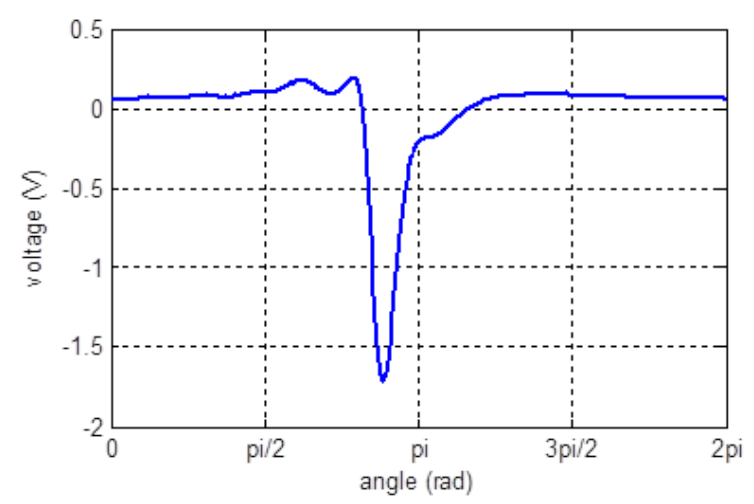

(c)

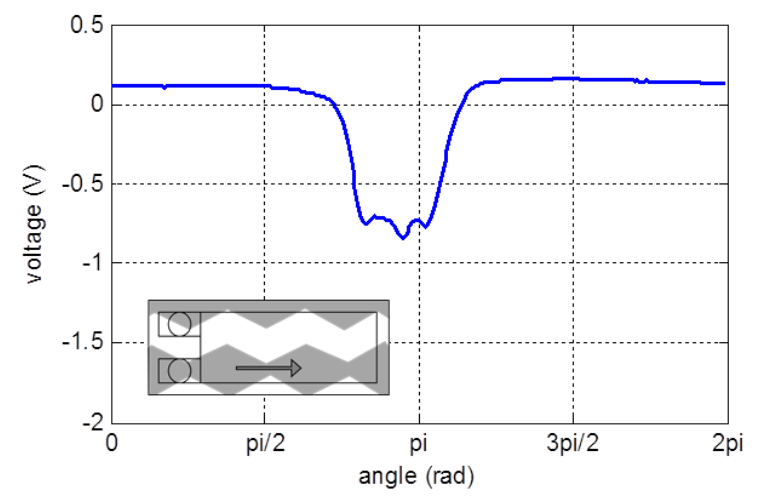

(e)

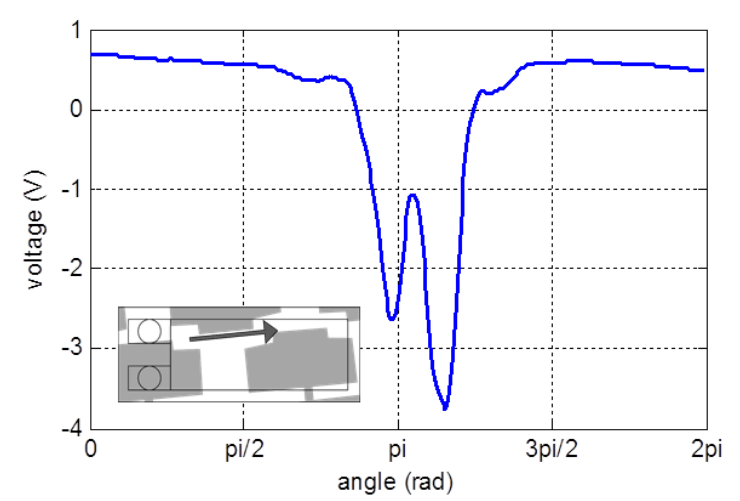

(b)

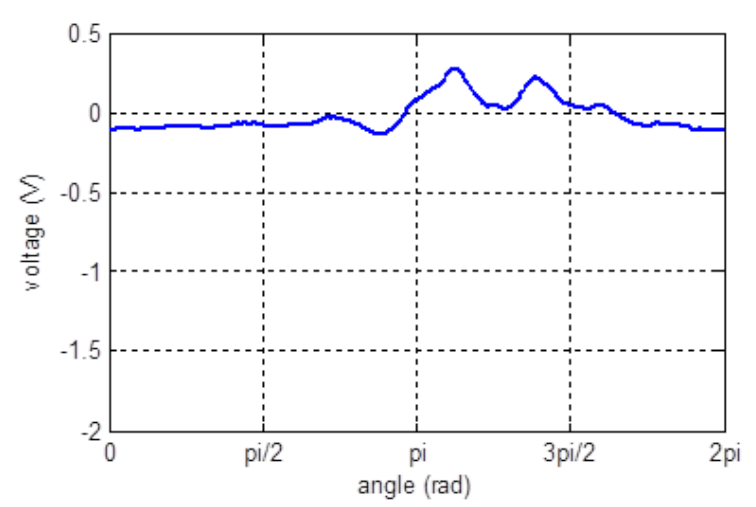

(d)

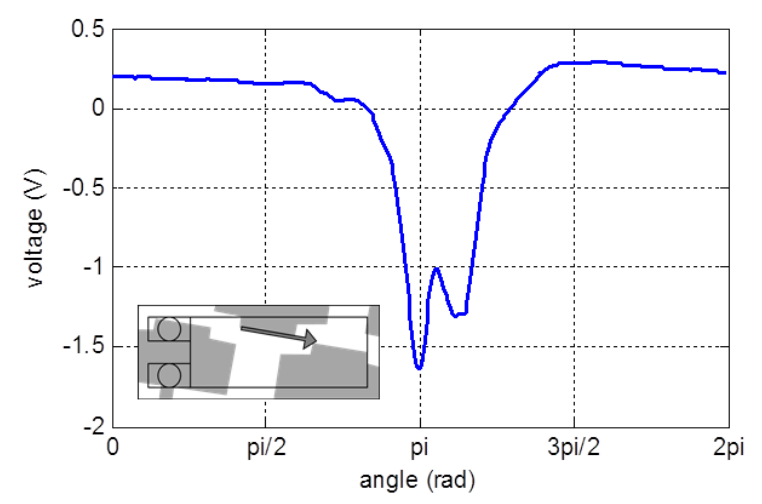

(f)

Note: The inset shows the location of the circumferential sensors along the tread pattern. The arrow shows the direction of the rotation, where the head of the arrow would be the first to enter the contact patch.

Figure 5.2: Representative curves, taken from $10 \mathrm{lb}$ payload, $100 \%$ power, on cement.

(a) Sensor 1 (unlaminated, circumferential), 6" tires, (b) sensor 1 (unlaminated, circumferential), 10" tires, (c) sensor 2 (unlaminated, radial), 6" tires, (d) sensor 2 (unlaminated, radial), 10" tires, (c) sensor 3 (laminated, circumferential), 6" tires, and (d) sensor 3 (laminated, circumferential), 10" tires. 
intervals of time. This means that we could expect the derivative of the curves measured here to match the approximate shape measured by Yi and Liang [73].

\subsubsection{Signal Amplitude}

One of the characteristics used to describe the signals was the amplitude. In the Figures 5.3, 5.4, and 5.5, the average amplitude for each test condition is compared as a bar graph. To determine the average amplitude, a representative trial was selected for each test condition. The amplitude of the signal during each rotation in the constant forward velocity region was calculated and the average value is presented in the figures. The individual values of amplitude for each rotation are shown as symbols on top of the bar graphs.

The amplitude is typically larger for all sensors with the $10 \mathrm{lb}$ payload compared to the $0 \mathrm{lb}$ payload. This is expected, because with increased load, there is increased deflection and normal load. No clear trend was seen in the amplitudes for tests conducted at different power levels. In general, when comparing matched test conditions from the 6 " tires and the 10" tires (sensors 1 and 3 only), the amplitude of the sensor signal was higher for the 10" tires.

In Section 3.2.2, it was explained that sensor 1 is an unlaminated film placed in a circumferential direction along the tire tread. It is expected that this sensor will be sensitive to both normal pressure in the contact patch and shear forces $F_{x}$ and $F_{y}$ that are transferred through the tire and adhesive. Sensor 2 is in the same location and is laminated. It was expected that the signal from sensor 2 would be most sensitive to normal pressure along the contact patch. Sensor 3 is unlaminated and is located on the sidewall. This placement was planned so that the sensor would measure sidewall deflection.

Comparing the amplitude of the sensor 1 measurements across different surfaces, the most pronounced differences can be seen with the 6" tires. Other than the $50 \%$ power at $0 \mathrm{lb}$ payload case, all other cases for this test condition show that the signal amplitude for sensor 1 is higher on cement than on ice. While the reasons for this trend cannot be confirmed, a theory is presented here. The differences seen may be caused by differences in the longitudinal force that is transferred through the tire tread. The maximum longitudinal

force, $F_{x \max }$, would be lower for the ice than the cement due to the lower coefficient of friction. If sensor 1 is sensitive to $F_{x}$ and the 6 " tires are slipping on ice but not on cement (and therefore have a lower $F_{x}$ value), then the signal would be lower for the ice than the cement. This is not supported by the speed results which showed that the wheel speed of the 6" tires with $10 \mathrm{lb}$ payload was actually slower on ice compared to cement. However, without knowledge of the vehicle velocity, the true slip value remains unknown.

The same trend lower sensor 1 amplitudes on ice is not seen for the 10" tires. This may be due to differences in how effectively the longitudinal force at the tire-ground contact patch is transferred through the tire tread and the adhesive to the sensor. This is supported by two observations: (1) the tread was thicker and stiffer on the 10" tires than the 6" tires and (2) the double sided tape used between the sensor and the tire did not adhere as strongly to the 10 " tires as it did to the 6" tires.

In general, sensor 1 had lower amplitude on sand than on cement for both tire sizes. With 


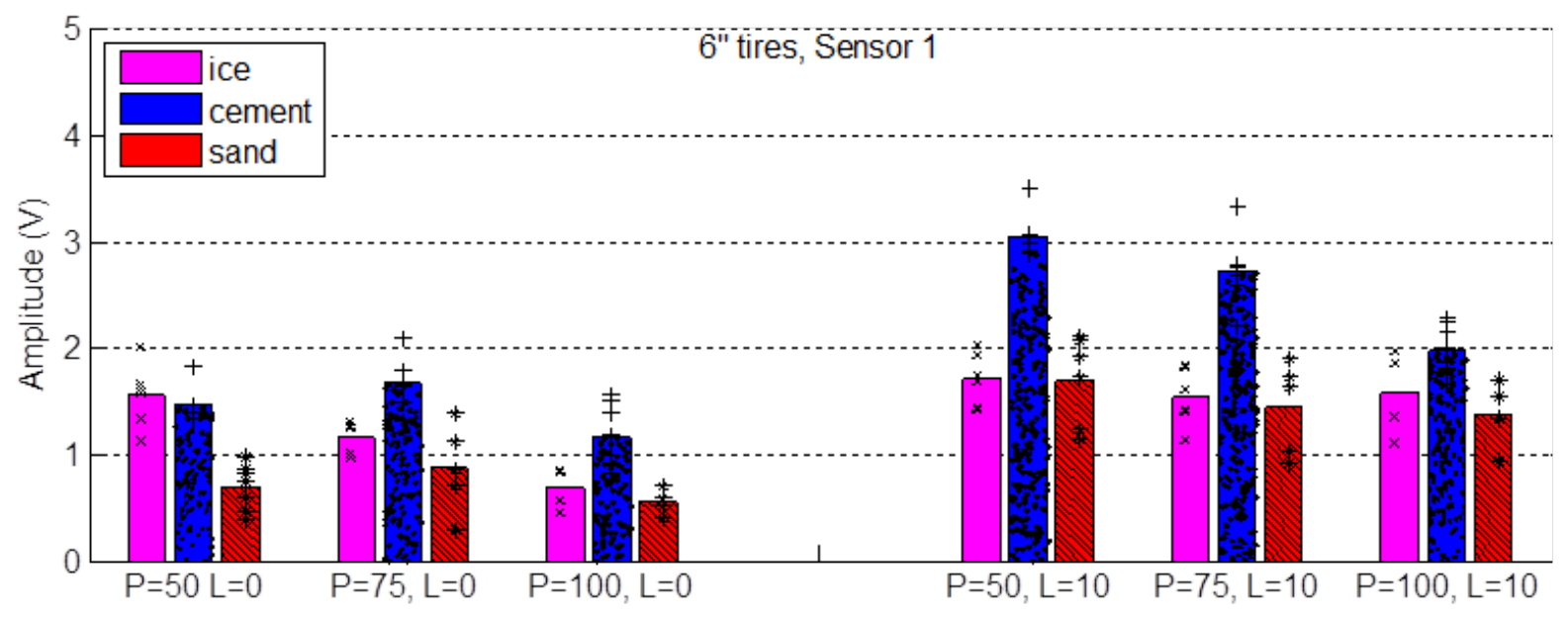

(a)

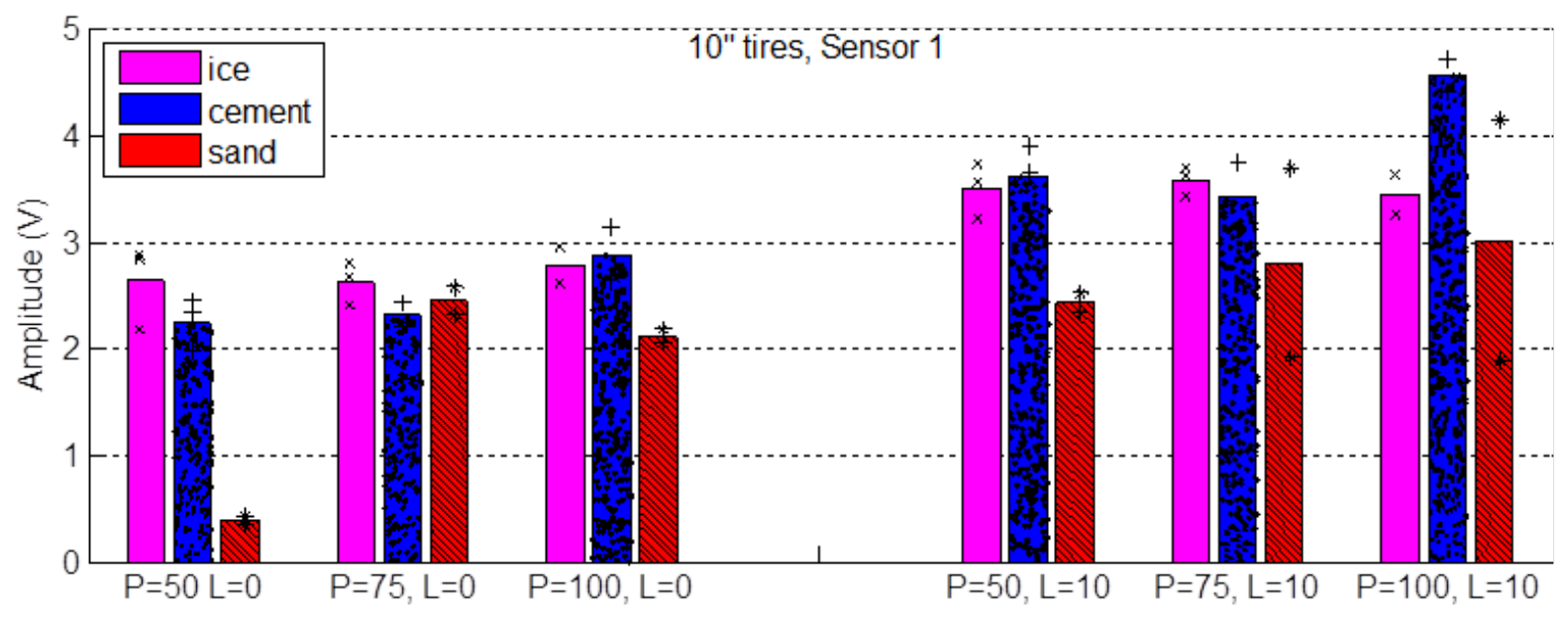

(b)

Figure 5.3: Bar graph of average maximum amplitude for sensor 1 (unlaminated, circumferential) for each test condition.

(a) 6" tires, (b) 10" tires 


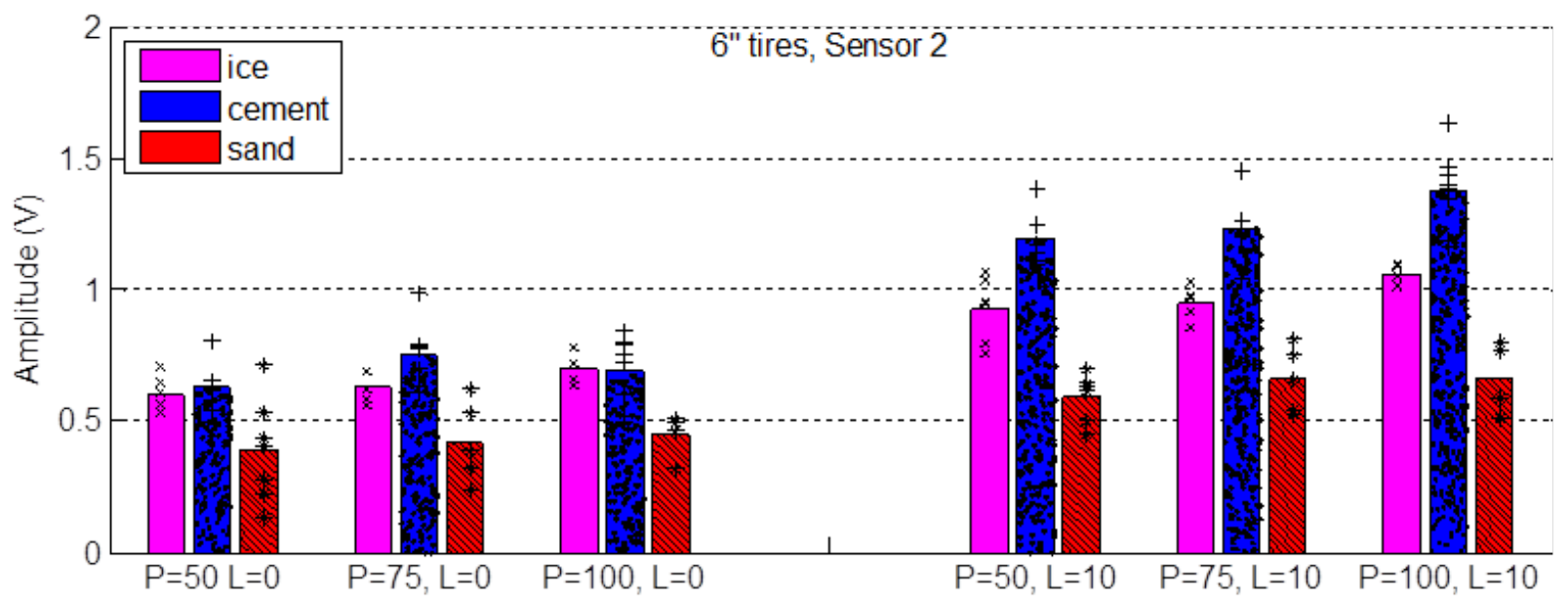

Figure 5.4: Bar graph of average maximum amplitude for sensor 2 (unlaminated, radial) for each test condition for the $6 "$ tires.

a larger contact patch on sand compared to cement or ice, the normal load is distributed across a wider area leading to a lower average pressure. From visual inspection, it was determined that the width of sensors was smaller than the the width of the contact patch on sand, but not on ice and cement. If the contact patch is wider than the sensor, then the total normal pressure on sensors located on the tread will be reduced.

For the 6" tires, the same trend between amplitudes for sensor 1 on cement and sand are seen for sensor 2 and to a lesser extent for sensor 3. This makes sense because it is believed that all of these sensors are sensitive to normal pressure which is in turn related to sidewall and tread deflection. No data was analyzed for sensor 2 on the 10" tires. For sensor 3 on the 10 " tires, the average amplitude was similar across test conditions and no consistent trend was seen.

\subsubsection{Signal Width}

The other characteristic used to describe the signals was the full width at half maximum (FWHM). In the Figures 5.6, 5.7, and 5.8, the average FWHM for each test condition is compared as a bar graph. The average FWHM was determined in the same manner that the average amplitude was determined, as described in Section 5.3.3.

For different applied power levels, little difference in the width is seen across the tests. The exception to this is sensor 1 for the 10" tires, seen in Figure 5.6a. There, a trend toward having a smaller width at higher power levels is seen, particularly on sand.

Between the three surfaces, the average FWHM is greatest on sand for all sensors and test conditions with the 6" tires and most sensors and test conditions with the 10" tires. The contact patch length is not expected to be substantially different on the ice compared to cement. The contact patch length on the sand is larger than on the hard surfaces. If 


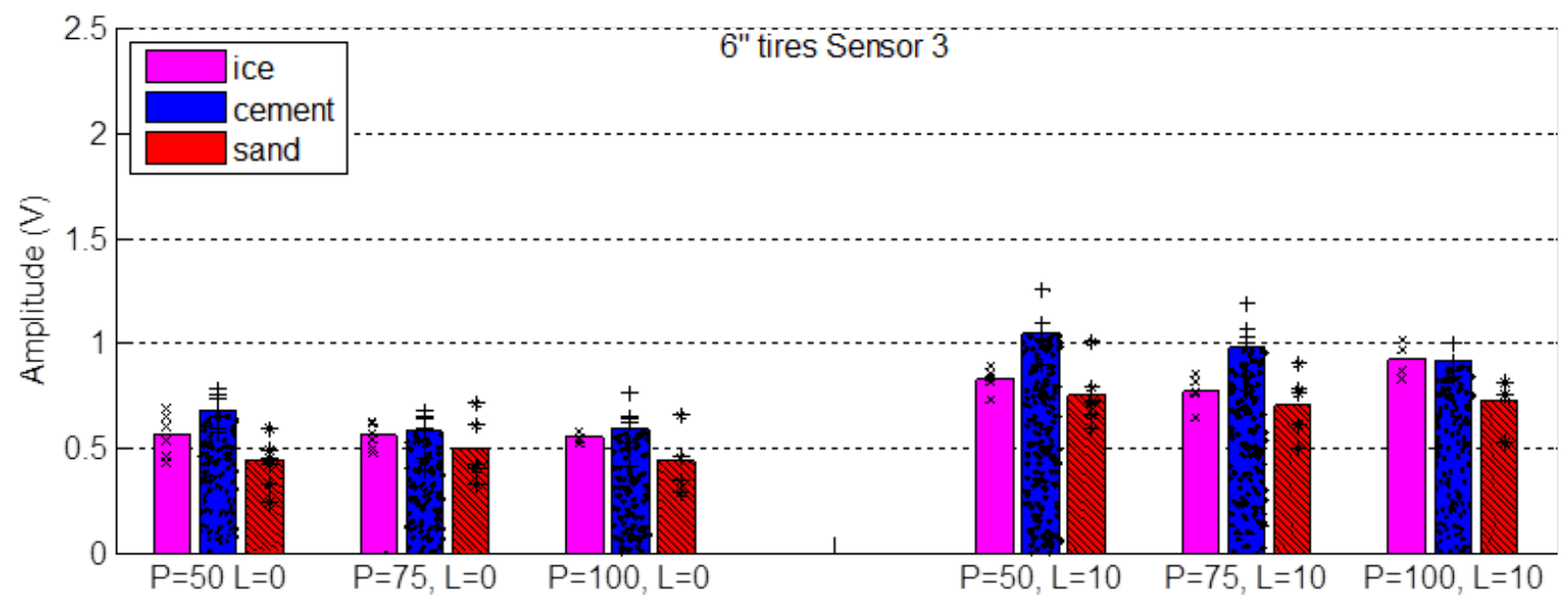

(a)

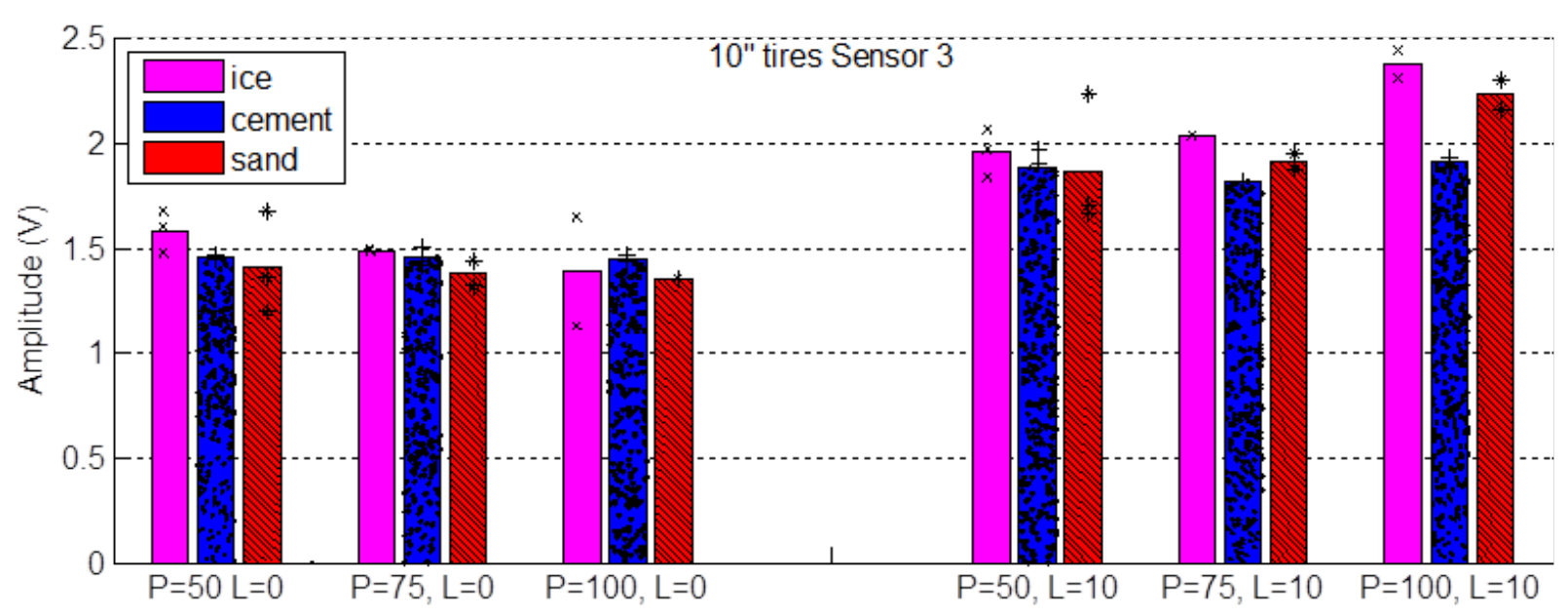

(b)

Figure 5.5: Bar graph of average maximum amplitude for sensor 3 (laminated, circumferential) for each test condition.

(a) 6" tires, (b) 10" tires 


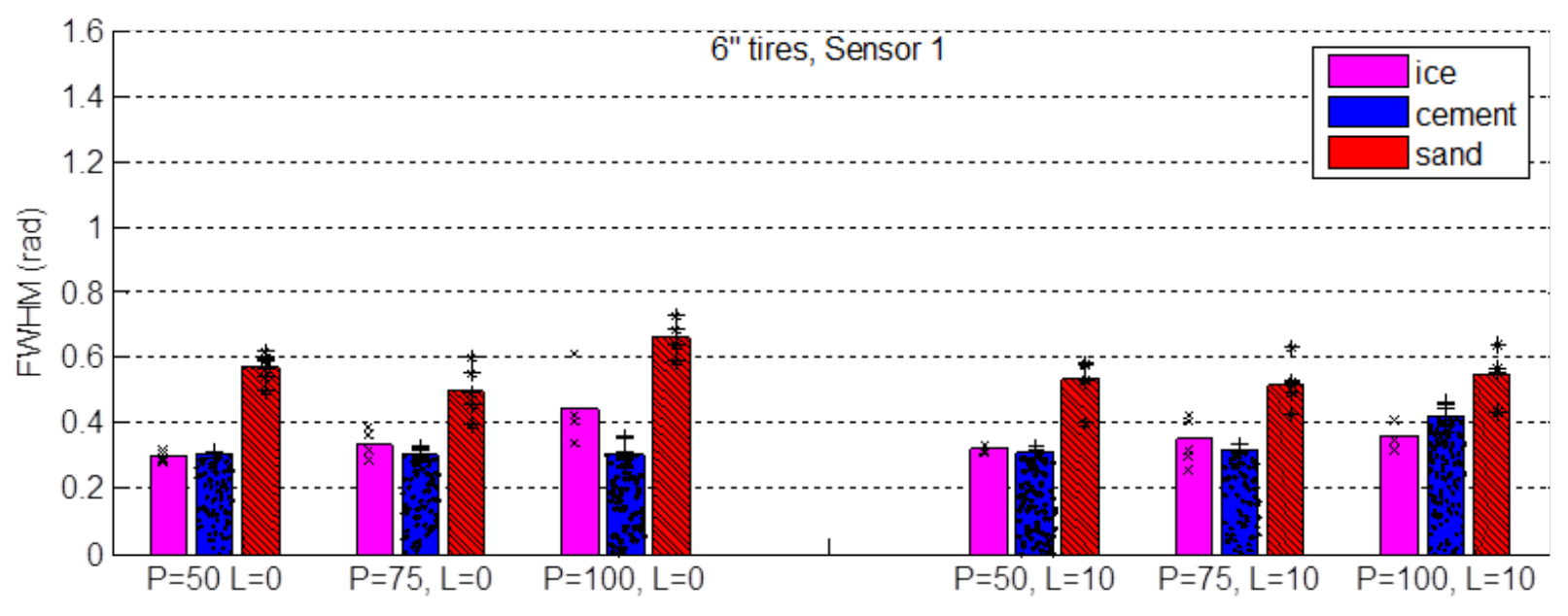

(a)

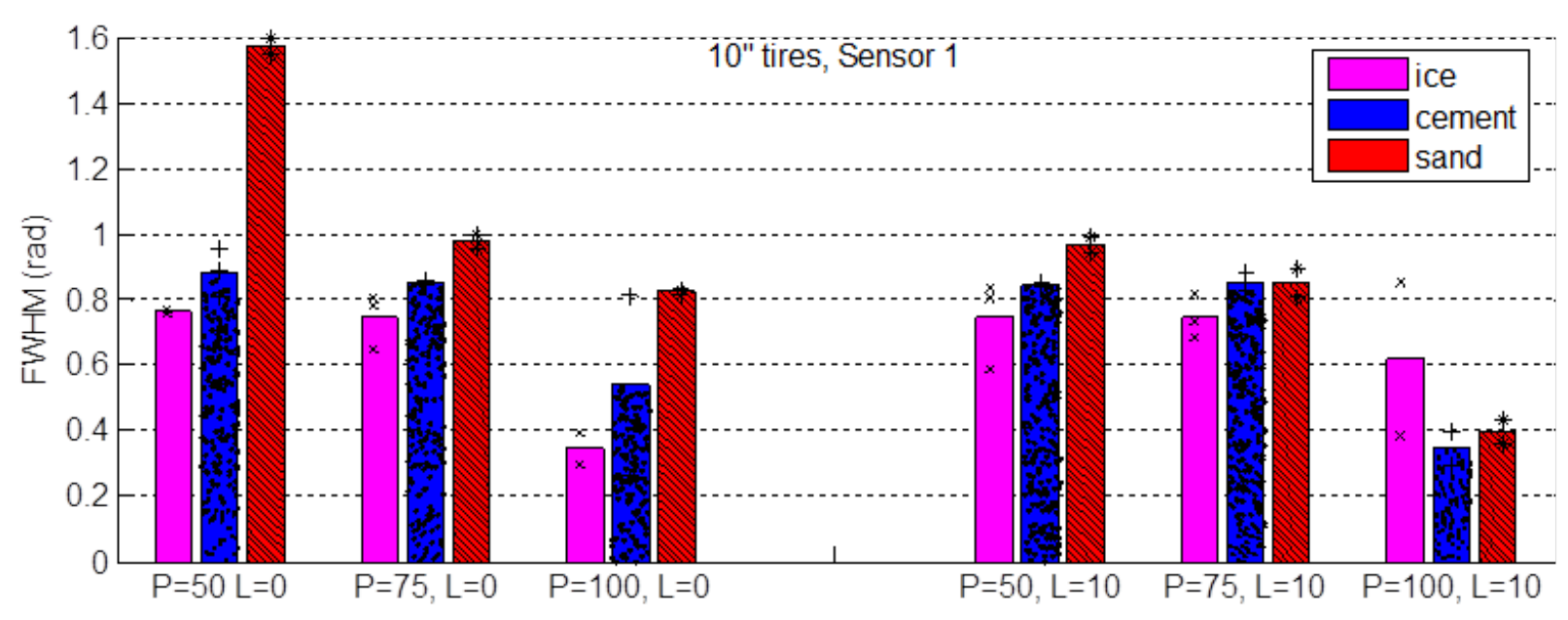

(b)

Figure 5.6: Bar graph of average sensor 1 FWHM for each test condition. (a) 6" tires, (b) 10" tires 


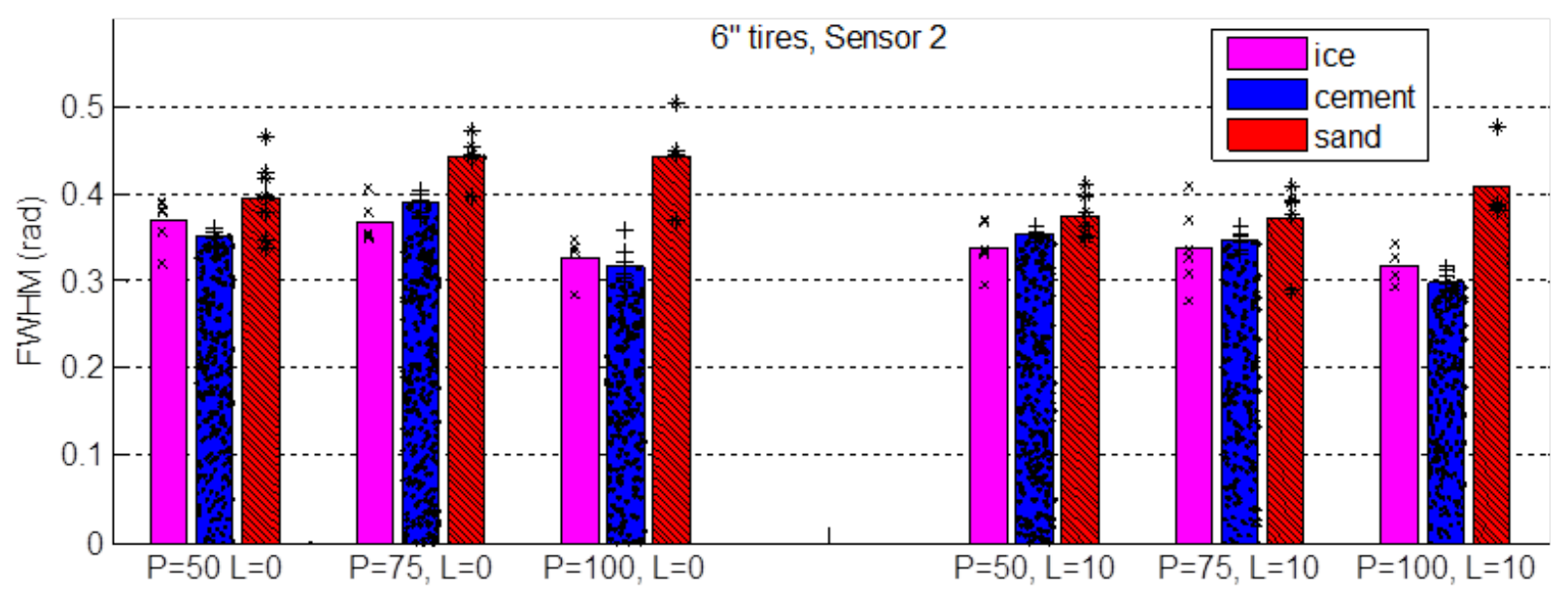

Figure 5.7: Bar graph of average sensor 2 FWHM for each test condition on 6" tires.

sensors 1 and 3 are sensitive to normal pressure and deflection and the normal pressure and deflection are greater within the contact patch than outside of the contact patch, then it would make sense that the width of the signal for sensors 1 and 3 would be larger on sand compared to ice and cement. If sensor 2 is sensitive to sidewall deflection and the sidewall deflects more when passing through the contact patch, it also makes sense that the width of the signal for sensor 2 would be larger on sand than on ice and cement. This is confirmed by the results for the 6 " tire that show a wider signal for all three sensors.

For the 10" tires, the results are more variable. As was seen in Figure 5.2, the sensor 1 and 3 signals for the 10" tires picked up a lot of variability due to the tread pattern. In some cases, it was seen that the algorithm for the full width at half maximum was measuring different characterizing different parts of the signal as the width. For example, if there was a very strong double peak phenomena and one of the peaks was much larger than the other, the width algorithm may only have registered the width of the first peak, rather than the width of the entire signal. This is what may have caused some measurements of the same test condition to be very different, for example the 10 " tires with $P=100 \%$ and $10 \mathrm{lb}$ payload, seen in Figure 5.6b.

\subsubsection{Summary of Results}

With the exception of sensor 2 on the 10" tires, all of the sensors measured signals in a consistent form throughout tests. The amplitude of the analyzed sensor measurements was greater for the cases with added payload, suggesting that the sensors were capable of measuring normal pressure or deflection.

A theory was presented to interpret the results that correlated well with the results for the 6" tires. A lower amplitude of the sensor 1 signal may indicate a lower tractive force 


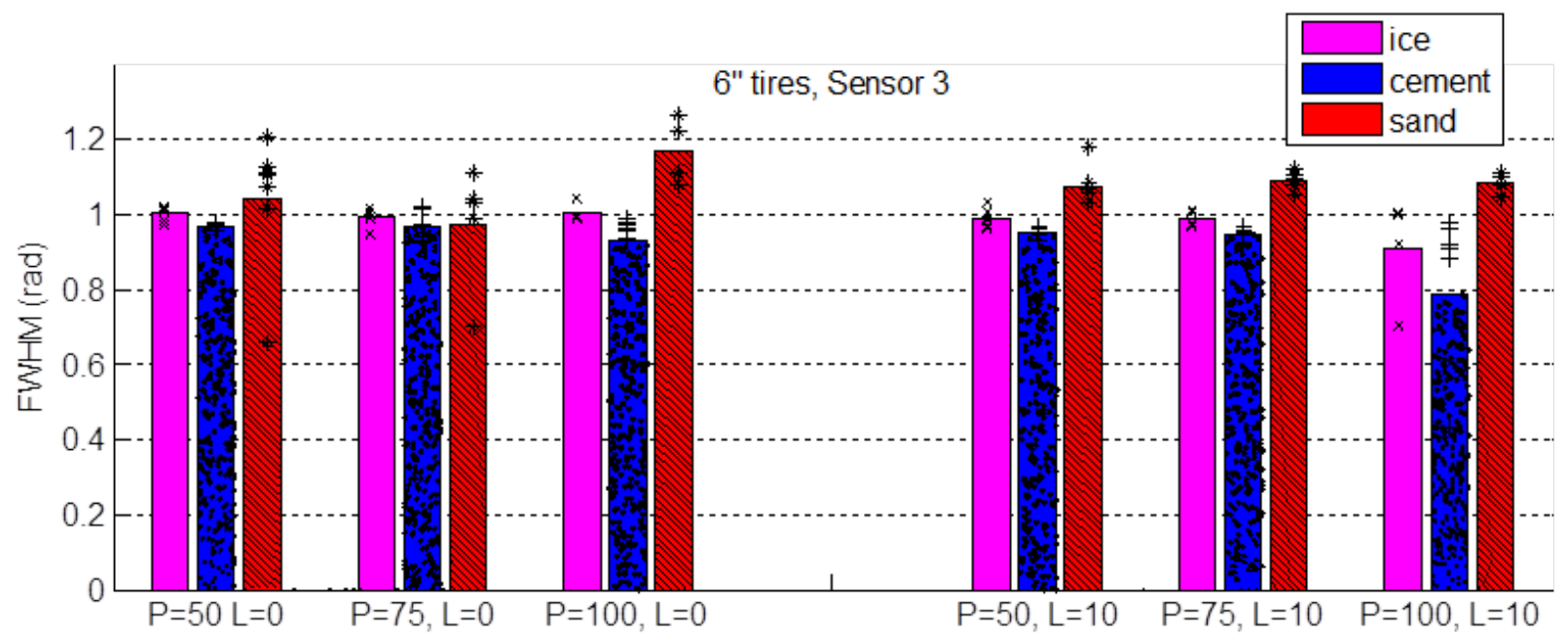

(a)

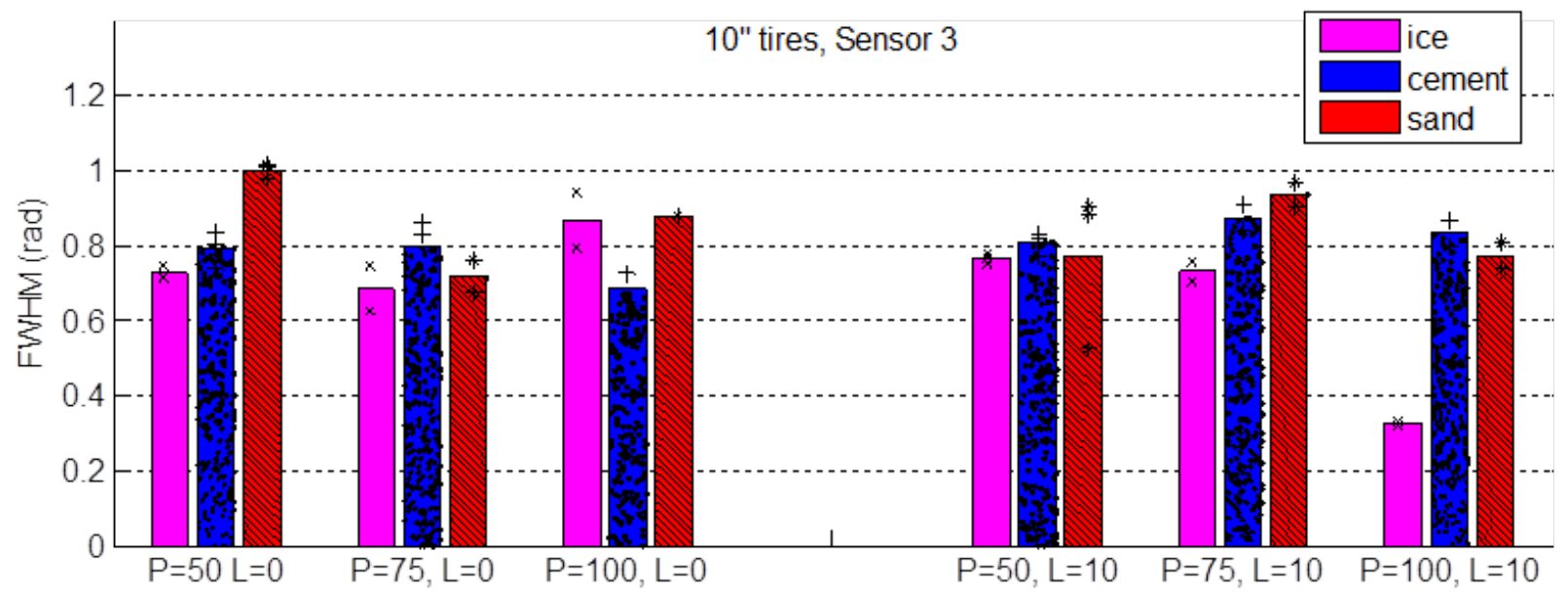

(b)

Figure 5.8: Bar graph of average sensor 3 FWHM for each test condition. (a) 6" tires, (b) 10" tires 
and/or a wider contact patch. To differentiate between these effects, the width of the sensor 1 signal can be examined. Lower tractive force will not change the width of the signal, but a larger contact patch will. Thus, from the amplitude and width of the sensor 1 measurements, characteristics of the ground surface can be determined.

For the 10" tires, none of the sensor placements picked up consistent trends between ice and cement. This may be due to the transfer of the shear force through the tire tread being reduced for the 10" tires compared to the 6" tires. Furthermore, the width measurements were not consistent with one another for the 10" tires. This may be due to the large size of the tread which in turn had a dramatic effect on the shape of the signal, making it difficult to characterize the width using a single simple algorithm.

\subsection{Limitations}

The assertions made in the preceding discussion are limited because no steps were taken to verify the tire deflections or directly measure the forces experienced by the tire. Furthermore, the level of slip is unknown because the linear vehicle velocity was not measured. The differences in the ability of the laminated and unlaminated sensors to measure normal and shear forces has not been quantified.

After testing was completed, it was determined that the sensors were not aligned properly with the circumferential direction along the tread. This further limits the ability to directly compare measurements taken from different tires and from the laminated and unlaminated sensors along the tread.

The relatively small number of surfaces tested limits the ability to generalize any observations to other types of terrain. Furthermore, the differences of the measured cone index for the prepared terrain demonstrate the lack of control over the sand test surface. Differences in parameters seen between tests on sand could be due to the differences in preparation. The tests for the 6" tires and the 10" tires on sand were conducted on different days. It was determined from visual inspection that the sand was more moist on the day of testing the 10 " tires. It must be considered that any differences seen between the 6" tires and the 10" tires on sand may be due to the difference in the moisture content rather than differences in the tires.

While the temperature of the outside surface of the tires was able to be measured between tests, it is not known how the heat transfers through the tires. 


\section{Chapter 6}

\section{Conclusion}

This chapter begins with an analysis of the progress toward the objectives originally presented in Section 1.2 and ends with suggestions of future work to be completed.

\subsection{Evaluation of Progress Toward Objectives}

In the following sections, the work completed and described in this paper is presented in the context of the four objectives of the study.

\subsubsection{Evaluation of Objective 1}

The first objective of the study was to, "Develop a low cost small wheeled mobile robot for intelligent tire testing". A semi-custom robot kit from SuperDroid Robots was selected, purchased, and assembled. The differential steered robot features two sets of pneumatic tube-type tires with 6" and 10" diameters. The robot can be controlled by either a handheld radio transmitter or by a pre-programmed script loaded onto the motor controller. There is space on the robot platform for a signal conditioner, DAQ, and laptop computer, which enable data collection for intelligent tire testing. The motor controller on the robot can record speed from two encoders placed on the motors and transmit this information to the laptop computer. Objective 1 was successfully met.

\subsubsection{Evaluation of Objective 2}

The second objective of this study was to, "Instrument the tires of the robot with sensors to characterize the tire-ground interaction". One tire of each of the two types tested was instrumented with three low cost piezoelectric sensors from Measurement Specialties. The three sensor configurations used were: 1) unlaminated circumferential (on the tread), 2) unlaminated, radial (on the sidewall), and 3) laminated, circumferential, (on the tread). The signal from the sensors was transferred from the tire through a slip ring, provided by Moog Components Group. The signal conditioning unit consisted of a charge 
amplifier followed by an inverting amplifier. The circuit transferred the charge accumulated on the surface of the piezoelectric sensor to a voltage that could be measured by the DAQ. It also elongated the time constant of the circuit, allowing for measurements of not purely strain changes. The signal conditioning circuit was constructed on a $2.5 " \times 2.5$ " prototype board and was shown to successfully reduce electronic noise from the motors and scale the signal output to be within the limits of the DAQ unit.

The results of the test procedure carried out suggest that the sensors were able to recognize components of the tire-ground interaction, however the exact interaction is now known. Therefore, some of the criterion for Objective 2 was met, while it is uncertain if it was fully met.

\subsubsection{Evaluation of Objective 3}

The third objective of this study was to, "Develop and implement a test procedure for using the robot with the sensors on off-road surfaces". A series of tests were planned and conducted to test the robot and the piezoelectric sensors in the tires. The test sequence consisted of straight forward movement at a set power level followed by a straight reverse movement at the same power level. This test sequence was written into a MicroBasic code that was loaded onto the Roboteq SDC2130 motor controller. The script allowed for the power sequence to be run at any one of three power levels. A robust and highly configurable LabView VI was designed to communicate with both the DAQ and the motor controller. Through LabView, data logging from the DAQ and the controller could be started and stopped together, in coordination with the power sequence running on the robot. The testing procedure was carried out on three surfaces: ice, polished cement, and sand, at two payloads: 0 lbs and $10 \mathrm{lbs}$, for two tire sizes.

The testing was successful and demonstrated the ability of the robotic test system to operate in a controlled manner on both hard and deformable surfaces while collecting data. The LabView VI can be used in future tests to communicate with the motor controller and/or the DAQ. The VI can be easily modified for use with other DAQ models or for recording data from additional channels. This test procedure was successful and can be easily reproduced for future tests, which indicates that objective 3 was met.

\subsubsection{Evaluation of Objective 4}

The fourth an final objective was to, "Investigate the possible information that can be gathered from these sensors regarding the tire-ground interaction". Data was successfully gathered from 5 of the 6 piezoelectric sensors. Sensor 2 on the 10 " tire had a very weak signal and it is unknown if this was due to a bad connection or whether it was reading a true phenomena. A qualitative analysis of the signals from each sensor was conducted and presented in this document. To characterize the signal from the sensor for each tire rotation in a quantitative way, two parameters were chosen: the amplitude and the full width at half maximum (FWHM). 
Differences in these parameters for the different payload levels were seen for most test conditions, suggesting that the sensors are picking up some useful information regarding load or tire deflection. For the different surfaces, more differences in amplitude and FWHM were seen for the 6" tires than the 10" tires.

An investigation was conducted into the use of piezoelectric sensors in the wheels of the test system. Results indicate that the sensors can be used to elucidate some information about the tire-ground interaction, but more work is needed to get specific ground parameters. The basic intent of objective 4 was met, but there is still work that can be done in this area.

\subsection{Recommendations for Future Work}

A test system has been created and the potential to use it as a platform for intelligent tire development has been demonstrated, but future studies can get more out of the system by obtaining more specific information about the operating parameters and test conditions. Ultimately, the information gathered from any tire sensors will be most useful as part of a network of sensors in the tire and on the robot itself. Additional sensors on the robot can be used in coordination with the tire sensors or can be used as part of a prototype system to validate measurements from the tires. Examples of other parameters about the robot that would be helpful to obtain in future tests are drawbar pull, vehicle speed, wheel speed of each of the four wheels, the precise energy consumption of each motor, and the absolute rotational position of each wheel.

Three sensor configurations were tested. The results suggest that the unlaminated sensor placed circumferentially along the tread may have the most potential to be used to characterize the tire-ground interaction. Future tests should include a sensor in this configuration. The other sensor configurations may be useful as part of a larger system of sensors.

Vehicle velocity and wheel slip are needed for validation and interpretation of piezoelectric sensor results. Johnson [29] also tried to use a LIDAR system previously developed by Ahmadian and Holton [5] to measure wheel slip. It was found that the measurements were degraded by noise due to attachment to the small robotic system. Future work is needed to identify a reliable method to measure wheel slip and/or vehicle velocity for small wheeled robots.

More tests need to be conducted to characterize the material and thermal properties of the tires. Tests should also be conducted to determine the precise response of each of the piezoelectric sensors types to strain in each principal direction. Once the properties of the sensors and the tires have been established, the sensor should be mounted in the tires and additional tests should be conducted to characterize the interaction between the piezoelectric sensors and the tires. These tests could include putting the tire on a hydraulic actuator or dynamometer to determine the response of the sensors to different stresses at the tire-ground interface.

In the study presented in this document, the sensors were attached to the tire with a piece of double sided tape. The tape did not adhere well to the inside surface of the tire, resulting in the sensors being moved out of their intended position when the tires were 
inflated. Furthermore, the poor adhesion may have affected the transfer for longitudinal forces from the tire-ground interface to the sensor placed along the tread. In future work with these tires and robots, the adhesion method should be improved. This may require modification of the inside surface of the tire (for example, smoothing it).

A pressure pad system made by TekScan is owned by the AVDL that could be used in future tests to validate assertions about the normal pressure distribution in the contact patch. This was attempted by Johnson [29] for a $7.4 \mathrm{~kg}$ robot, but it was found that pad was not sensitive enough. The robot used in our study has a minimum weight of $13.6 \mathrm{~kg}$.

The analyses conducted in the present study were categorical in nature (ex. low vs high payload, soft vs hard surface) rather than quantitative. Future expansion of the test matrix should include a wider range of deformable surfaces that are very controlled in nature. Other commercially available tires could be selected for testing that vary from one another in only a few measurable parameters as was done in Taylor [65] for larger tires. These changes may allow the characteristics of the measured signals to be directly correlated to certain tire or surface parameters.

The test matrix can be expanded to include controlled turning maneuvers. In order to do this, it may be preferable to introduce a second motor controller so that each of the four motors can monitored and powered independently. If turning maneuvers are tested, placement of sensors in the lateral direction along the tread should be considered.

Continuing work with this test system has already begun with efforts to implement uniaxial and/or triaxial accelerometers into the tire treads. Once the characteristics of the tire-ground interaction that can be determined by the accelerometers and/or the piezoelectric sensors are identified, the sensor measurements will be implemented as part of a control system to improve vehicle mobility. 
Appendices 


\section{Appendix A}

\section{Connectors Used on Robot}

The selection of appropriate connectors for an signal connections is very important in any test system. The connectors must be sized appropriately for the diameter of the wire attached, be rated for the expected current, and must have a noise level acceptable for the application. Because of this importance, the connectors added to the robot are listed in Table A.1.

Table A.1: Connectors added to the robot test system.

\begin{tabular}{llll}
\hline Location and Type & Brand & Line & Series \\
\hline Piezo to Slip Ring & & & \\
Receptable & Molex & Micro-Fit & 43645 \\
Female Contact & Molex & Micro-Fit & 43030 \\
Plug & Molex & Micro-Fit & 43640 \\
Male Contact & Molex & Micro-Fit & 43031 \\
Slip Ring to Board & & & \\
Receptable & Molex & Micro-Fit & 43645 \\
Female Contact & Molex & Micro-Fit & 43030 \\
Right Angle Plug & Molex & Micro-Fit & 43650 \\
Encoder & & & \\
Receptable & Molex & Micro-Fit & 43645 \\
Female Contact & Molex & Micro-Fit & 43030 \\
Plug & Molex & Micro-Fit & 43640 \\
Male Contact & Molex & Micro-Fit & 43031 \\
Motor Cable & & & \\
Receptacle & Molex & Mini-Fit Jr & 5557 \\
Female Contact & Molex & Mini-Fit Jr & 5556 \\
Plug & Molex & Mini-Fit Jr & 5559 \\
Male Contact & Molex & Mini-Fit Jr & 5558 \\
\hline
\end{tabular}




\section{Appendix B}

\section{LabView Virtual Instrument}

The front panel for the custom built LabView VI is shown in Figure B.1. 


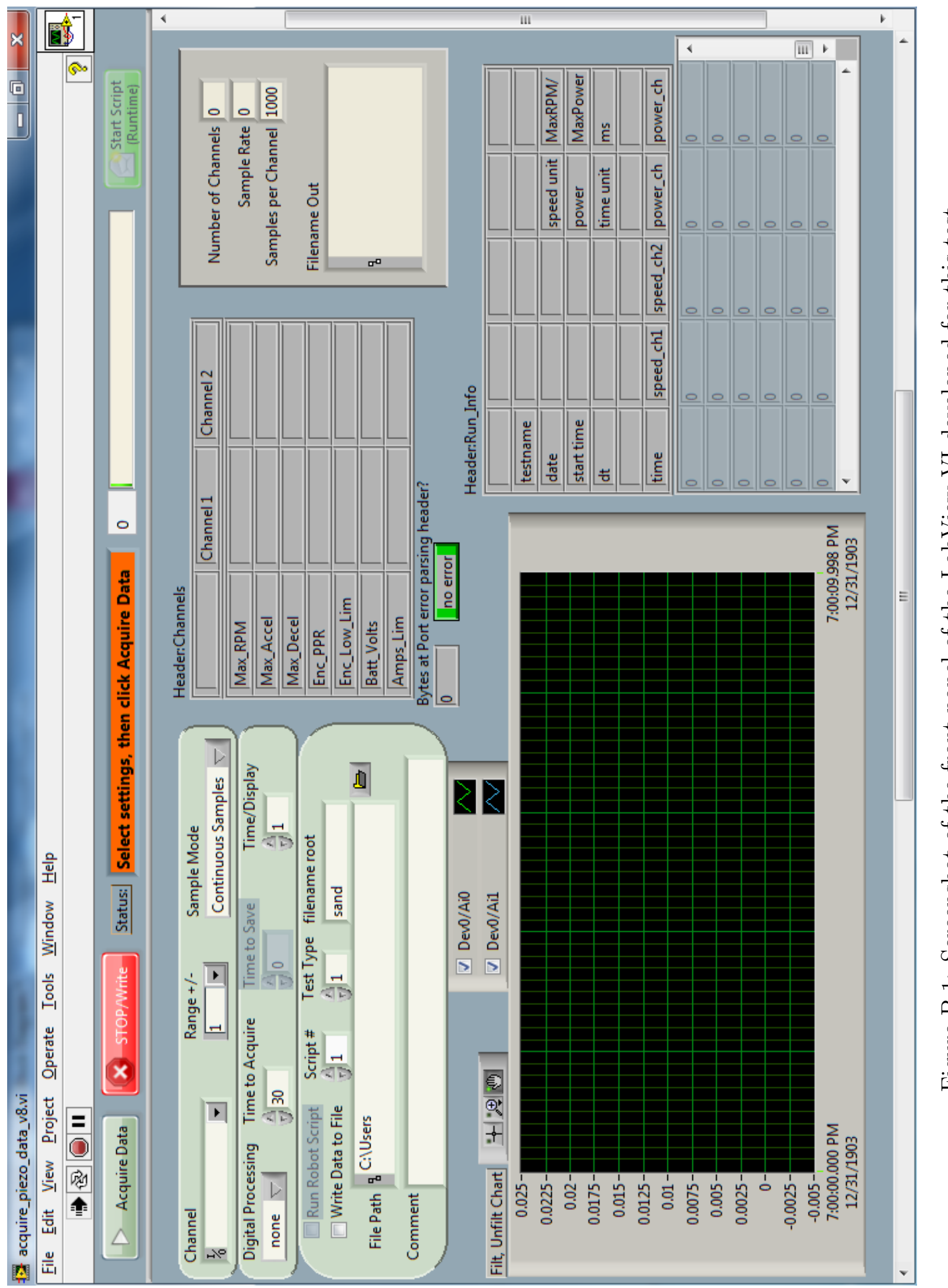

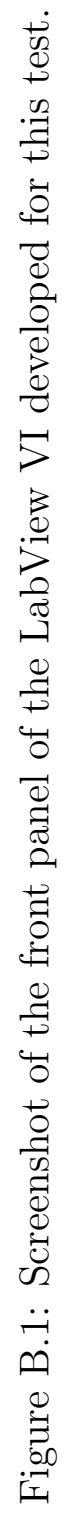




\section{Appendix $\mathrm{C}$}

\section{MicroBasic Script for 6" Tires}

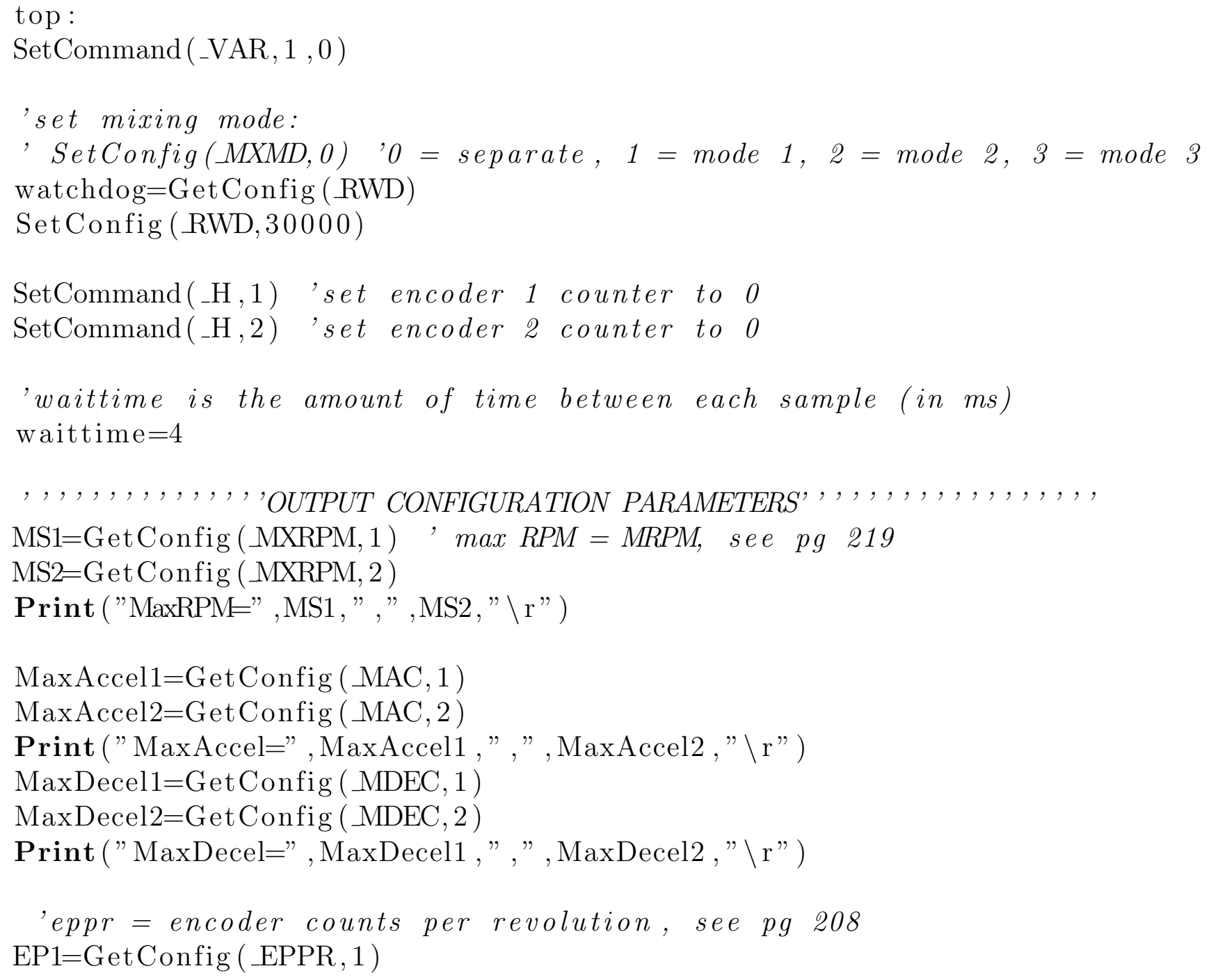


$\mathrm{EP} 2=$ GetConfig $($ EPPR, 2$)$

Print ("EncPPR=",EP1, , , ,EP2, "\r")

EHL1=GetConfig (_ELL, 1) 'encoder high limit

EHL2=GetConfig ( _ELL , 2)

Print ("EncLimits=",ELL1," ," ELL2,"\r")

bvolts $1=$ GetValue $\left(\_\mathrm{V}, 1\right)$

bvolts $2=$ GetValue $\left(\_\mathrm{V}, 2\right)$

Print ("Batt=", bvolts1,", , bvolts2, "\r")

ALim1=GetConfig $($ ALIM, 1$)$

ALim2=GetConfig ( ALIM , 2)

Print ("Alim=",ALIM1,", , ALIM2," \r")

'WAIT FOR START SIGNAL FROM COMPUTER

While GetValue $($ VAR, 1$)=0$

wait (10)

End While

Print ("started $\backslash n ")$

wait (waittime)

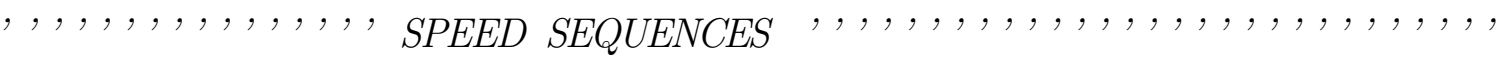

'in open loop, mixing mode 1, "go" for channel 1 sets motor command 1

'for state, 1 means timer has reached zero, 0 means timer is running

GoSub Baseline

If GetValue $($ VAR, 1$)=1$

SetTimerCount $(2,12500)$

'value is part of 1000, for open loop this is the relative power, 'for closed loop this is the relative speed

SetCommand (_GO, 1, 500)

Do

GoSub Output

Wait (waittime)

Loop While GetTimerState $(2)<1$

GoSub Baseline

GoSub Baseline 
SetTimerCount $(2,12500)$

SetCommand(_GO, 1, -500)

Do

GoSub Output

Wait (waittime)

Loop While GetTimerState $(2)<1$

ElseIf GetValue(_VAR, 1) $=2$

SetTimerCount $(2,7000)$

SetCommand(_GO, 1, 750)

Do

GoSub Output

Wait (waittime)

Loop While GetTimerState $(2)<1$

GoSub Baseline

GoSub Baseline

SetTimerCount $(2,7000)$

SetCommand(_GO, 1, -750)

Do

GoSub Output

Wait (waittime)

Loop While GetTimerState $(2)<1$

ElseIf GetValue(_Var, 1$)=3$

SetTimerCount $(2,5000)$

SetCommand(_GO, 1, 1000)

Do

GoSub Output

Wait (waittime)

Loop While GetTimerState $(2)<1$

GoSub Baseline

GoSub Baseline

SetTimerCount $(2,5000)$

SetCommand(_GO, 1, - 1000)

Do

GoSub Output

Wait (waittime)

Loop While GetTimerState $(2)<1$ 


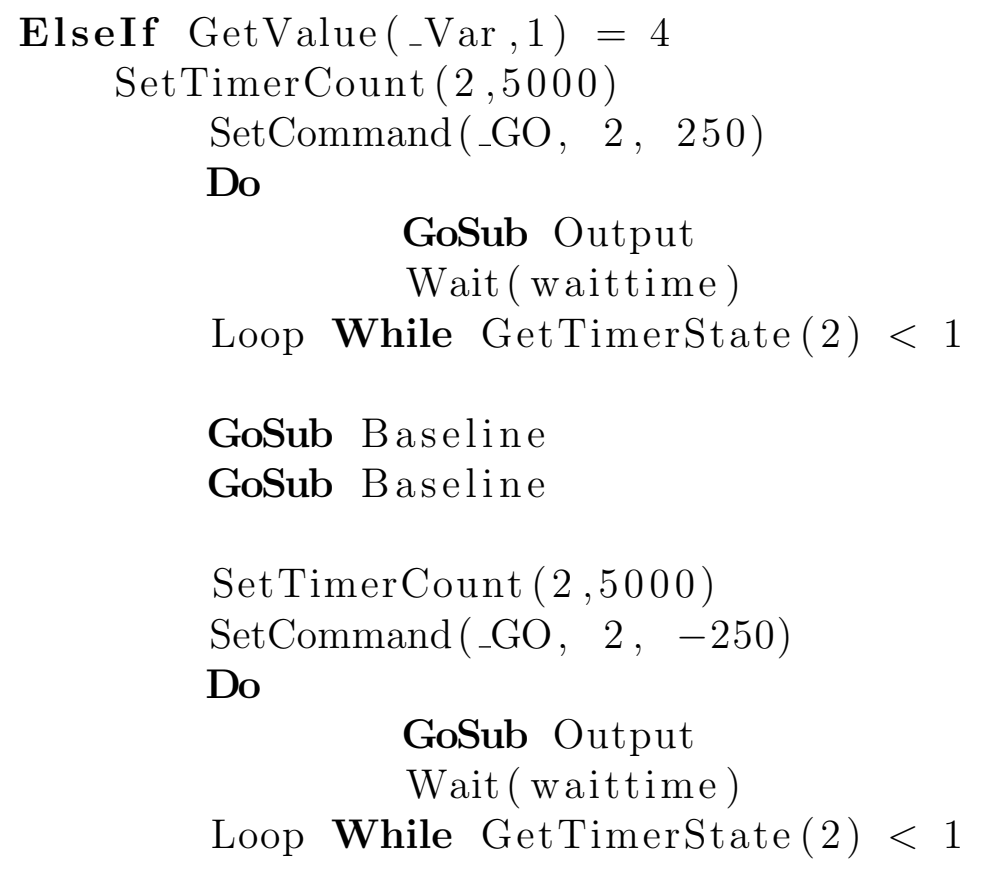

\section{End If}

GoSub Baseline

\section{ENDING OF SCRIPT}

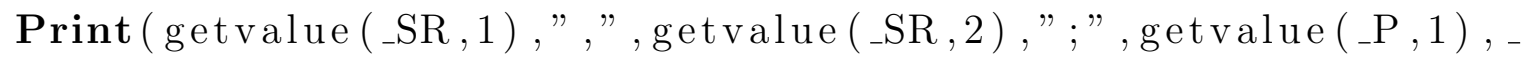

$$
\text { ", , getvalue (_P }, 2), " \text {; \nSTOP } \backslash n ")
$$

SetCommand (_GO, 1, 0)

SetConfig (RWD, watchdog)

\section{Terminate}

\section{Output:}

$$
\begin{aligned}
& \text { '32 characters per } 1 \mathrm{~ms} \text { time slot. } \\
& \text { 'Output Speed Values } \\
& \text {,'S }=\text { speed in } R P M \\
& ,{ }_{-} S R=\text { speed as } 1 / 1000 \text { of max } \\
& { }_{-} A=\text { motor amps }
\end{aligned}
$$

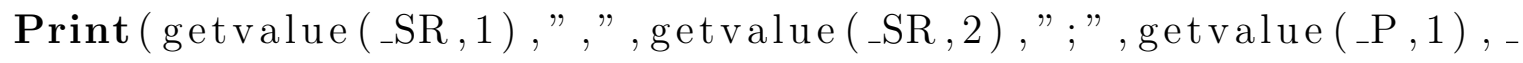




$$
\text { ", , getvalue (_P , 2), ; \\”) }
$$

\section{Return}

Baseline:

SetTimerCount $(1,2000)$

SetCommand (_GO, 1, 0)

SetCommand (_GO, 2, 0)

SetCommand (_H , 1)

SetCommand (_H, 2)

Do

GoSub Output

Wait (waittime)

Loop While GetTimerState $(1)<1$

\section{Return}




\section{Appendix D}

\section{MicroBasic Script for 10" Tires}

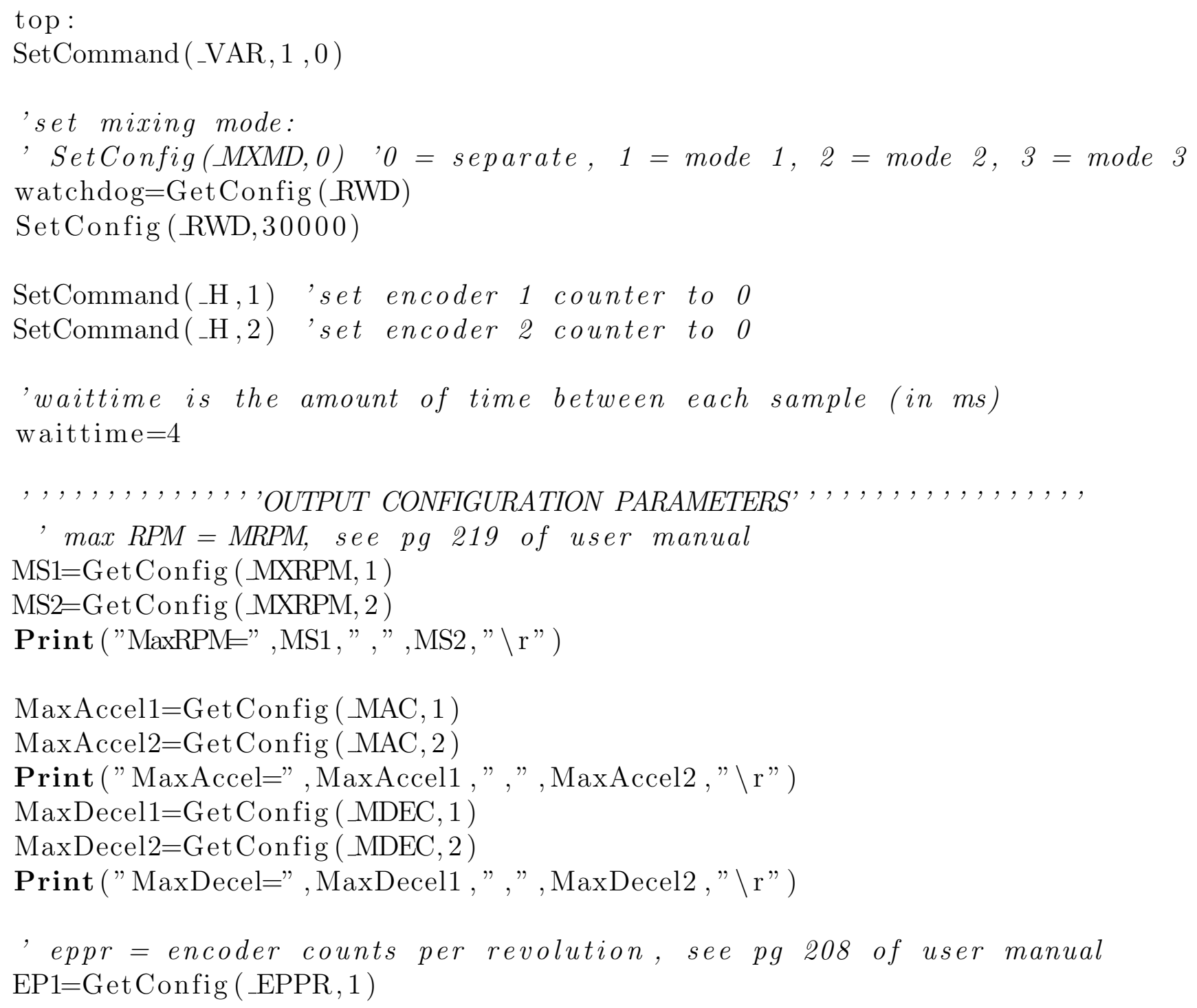


$\mathrm{EP} 2=$ GetConfig $($ EPPR, 2$)$

Print ("EncPPR=",EP1, , , ,EP2, "\r")

EHL1=GetConfig (_ELL, 1) 'encoder high limit

EHL2=GetConfig ( _ELL , 2)

Print ("EncLimits=",ELL1," ," ELL2,"\r")

bvolts $1=$ GetValue $\left(\_\mathrm{V}, 1\right)$

bvolts $2=$ GetValue $\left(\_\mathrm{V}, 2\right)$

Print ("Batt=", bvolts1,", , bvolts2, "\r")

ALim1=GetConfig $($ ALIM, 1$)$

ALim2=GetConfig ( ALIM , 2)

Print ("Alim=",ALIM1,", , ALIM2," \r")

', , , , , , , , , , ,WAIT FOR START SIGNAL FROM COMPUTER

While GetValue $($ VAR, 1$)=0$

wait (10)

End While

Print ("started $\backslash n ")$

wait (waittime)

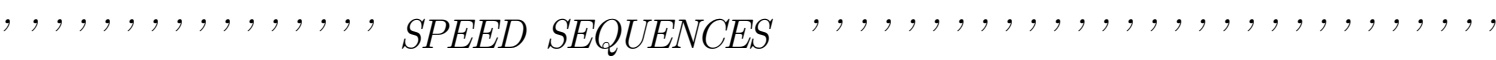

'in open loop, mixing mode 1, "go" for channel 1 sets motor command 1

'for state, 1 means timer has reached zero, 0 means timer is running

GoSub Baseline

If GetValue $($ VAR, 1$)=1$

SetTimerCount $(2,7000)$

'value is part of 1000, for open loop this is the relative power, 'for closed loop this is the relative speed

SetCommand(_GO, 1, 500)

Do

GoSub Output

Wait (waittime)

Loop While GetTimerState $(2)<1$

GoSub Baseline

GoSub Baseline 
SetTimerCount $(2,7000)$

SetCommand(_GO, 1, -500)

Do

GoSub Output

Wait (waittime)

Loop While GetTimerState $(2)<1$

ElseIf GetValue(_VAR, 1) $=2$

SetTimerCount $(2,4000)$

SetCommand(_GO, 1, 750)

Do

GoSub Output

Wait (waittime)

Loop While GetTimerState $(2)<1$

GoSub Baseline

GoSub Baseline

SetTimerCount $(2,4000)$

SetCommand(_GO, 1, -750)

Do

GoSub Output

Wait (waittime)

Loop While GetTimerState $(2)<1$

ElseIf GetValue(_Var, 1$)=3$

SetTimerCount $(2,3000)$

SetCommand(_GO, 1, 1000)

Do

GoSub Output

Wait (waittime)

Loop While GetTimerState $(2)<1$

GoSub Baseline

GoSub Baseline

SetTimerCount $(2,3000)$

SetCommand(_GO, 1, -1000)

Do

GoSub Output

Wait (waittime) 
Loop While GetTimerState $(2)<1$

ElseIf GetValue (_Var, 1$)=4$

SetTimerCount $(2,3000)$

SetCommand (_GO, 2, 250)

Do

GoSub Output

Wait (waittime)

Loop While GetTimerState $(2)<1$

GoSub Baseline

GoSub Baseline

SetTimerCount $(2,3000)$

SetCommand(_GO, 2, -250)

Do

GoSub Output

Wait (waittime)

Loop While GetTimerState $(2)<1$

\section{End If}

GoSub Baseline

Print (getvalue (_SR , 1) , , , , getvalue (_SR , 2) , ; ; , getvalue (_P , 1) , _

$$
\text { ", ", getvalue (_P , 2), "; \nSTOP \n") }
$$

SetCommand (_GO, 1, 0)

SetCommand (_GO, 2, 0)

SetConfig (RWD, watchdog)

\section{Terminate}

Output:

$$
\begin{aligned}
& \text { '32 characters per } 1 \mathrm{~ms} \text { time slot. } \\
& \text { 'Output Speed Values } \\
& ,-S=\text { speed in RPM } \\
& ,-S R=\text { speed as } 1 / 1000 \text { of max } \\
& , A=\text { motor amps }
\end{aligned}
$$




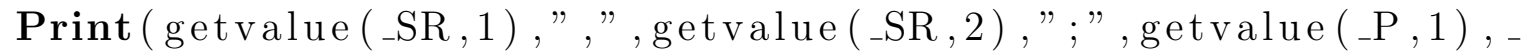
", , getvalue (_P , 2), " ; \n")

\section{Return}

\section{Baseline :}

SetTimerCount $(1,2000)$

SetCommand (_GO, 1, 0)

Do

GoSub Output

Wait (waittime)

Loop While GetTimerState $(1)<1$

SetCommand (_H, 1)

SetCommand (_H, 2)

\section{Return}




\section{Bibliography}

[1] APOLLO: Intelligent Tyre Systems - State of the Art and Potential Technologies. Deliverable D7. 2003.

[2] APOLLO: Final Report Including Technical Implementation Plan (Annex) Deliverable 22/23. 2005.

[3] 3M. Scotch Removable Double Sided Tape, $6673 / 4$ in $\mathrm{x} 400$ in, 2013. URL http://solutions.3m.com/wps/portal/3M/en_US/Marine/ Home/Products/Catalog/?PC_7_RJH9U5230GE3E02LECIE20S4K7000000_nid= 1TG0F7QJPKgsN4BX5SJJFCg1NM92GWTM31bl.

[4] 3M Industrial Adhesives and Tapes Division. Repositional Tapes, Datasheet, 2011. URL http://multimedia.3m.com/mws/mediawebserver? 66666UuZjcFSLXTtlxTV5XM6EVuQEcuZgVs6EVs6E666666--.

[5] M. Ahmadian and C. Holton. US Patent 7,705,972 B2. Doppler Sensor For the Derivation of Torsional Slip, Friction and Related Parameters, 2010.

[6] American Society of Agricultural and Biological Engineers. Procedures for Using and Reporting Data Obtained with the Soil Cone Penetrometer. ASABE Standards, ASAE EP542:1053-1055, 2006.

[7] Analog Devices. Op Amp Inputs, Outputs, Single-Supply, and Rail-to-Rail Issues. pages $1-12,2009$.

[8] ASTM. Standard Test Method for Using a Horizontal Pull Slipmeter (HPS). F609-05: $1-3,2013$.

[9] E. Bartolome. Signal Conditioning for Piezoelectric Sensors. Technical Report Q1, Texas Instruments, Dallas, TX, 2010. URL http://www.ti.com/lit/an/slyt369/slyt369. pdf.

[10] B. Blixhaven and T. Kvisteroey. US Patent 7,523,656 B1. Tire Sensor System and Monitoring Method, 2009. 
[11] F. Cheli, E. Leo, S. Melzi, and E. Sabbioni. On the Impact of Smart Tyres On Existing ABS/EBD Control Systems. Vehicle System Dynamics, 48(Supplement 1):255-270, Dec. 2010.

[12] O. Chuy Jr, E. G. Collins Jr, W. Yu, and C. Ordonez. Power Modeling of a Skid Steered Wheeled Robotic Ground Vehicle. In IEEE International Conference on Robotics and Automation, pages 4118-4123, Kobe, Japan, May 2009. IEEE.

[13] L. Ding, Z. Deng, H. Gao, K. Nagatani, and K. Yoshida. Planetary Rovers Wheel-Soil Interaction Mechanics: New Challenges and Applications for Wheeled Mobile Robots. Intelligent Service Robotics, 4(1):17-38, Dec. 2010.

[14] E. M. DuPont, C. a. Moore, E. G. Collins, and E. Coyle. Frequency Response Method for Terrain Classification in Autonomous Ground Vehicles. Autonomous Robots, 24(4): 337-347, Jan. 2008.

[15] M. Ekdahl, S. Nord, M. Kohler, T. Husemann, and C. Hartweg. D4: User Needs, Application Scenarios and System Requirements. Technical report, 2006.

[16] G. Erdogan. New Sensors and Estimation Systems for the Measurement of Tire-Road Friction Coefficient and Tire Slip Variables. Doctoral thesis, University of Minnesota, 2009.

[17] G. Erdogan, L. Alexander, and R. Rajamani. A Novel Wireless Piezoelectric Tire Sensor for the Estimation of Slip Angle. Measurement Science and Technology, 21(1):015201, Jan. 2010.

[18] G. Erdogan, L. Alexander, and R. Rajamani. Estimation of Tire-Road Friction Coefficient Using a Novel Wireless Piezoelectric Tire Sensor. IEEE Sensors Journal, 11(2): 267-279, Feb. 2011.

[19] S. C. Ergen, A. Sangiovanni-Vincentelli, X. Sun, R. Tebano, S. Alalusi, G. Audisio, and M. Sabatini. The Tire as an Intelligent Sensor, IEEE Council on Electronic Design Automation, 2009.

[20] Gears Educational Systems. The Surface Mobility Platform, 2012. URL http://www. gearseds.com/surface_mobility_platform.html.

[21] J. M. Giustino. System and Method for Predicting Tire Forces Using Tire Deformation Sensors, Dec. 2000.

[22] F. M. Gondal. Embedded Wireless Sensor Network for Aircraft/Automobile Tire Structural Health Monitoring. Master's thesis, Virginia Tech, Blacksburg, Va, 2007.

[23] H. Gu, Y. Zhao, and M. L. Wang. A Wireless Smart PVDF Sensor For Structural Health Monitoring. Structural Control and Health Monitoring, 12(3-4):329-343, July 2005. 
[24] F. Gustafsson. Slip-Based Tire-Road Friction Estimation. Automatica, pages 1-29, 1997.

[25] K. Iagnemma, S. Kang, H. Shibly, and S. Dubowsky. Online Terrain Parameter Estimation for Wheeled Mobile Robots With Application to Planetary Rovers. IEEE Transactions on Robotics, 20(5):921-927, Oct. 2004.

[26] ICT International Pty Ltd. CP40II Cone Penetrometer Operation Manual. Armidale, NSW, Australia, 1.0 edition.

[27] G. Ishigami, G. Kewlani, and K. Iagnemma. Predictable Mobility. IEEE Robotics \&6 Automation Magazine, pages 61-70, Dec. 2009.

[28] R. N. Jazar. Tire Dynamics. In Vehicle Dynamics: Theory and Application, chapter 3, pages 95-163. Springer US, Boston, MA, 2008.

[29] C. P. Johnson. Comparative Analysis of Lightweight Robotic Wheeled and Tracked Vehicle. Master's thesis, Virginia Polytechnic Institute and State University, 2012.

[30] J. Karki. Signal Conditioning Piezoelectric Sensors. Technical Report SLOA033A, Texas Instruments Inc, Dallas, TX, 2000.

[31] A. Kay. Analysis and Measurement of Intrinsic Noise in Op Amp Circuits. Technical report, Texas Instruments Inc, Dallas, TX.

[32] G. Krick. Radial and Shear Stress Distribution Beneath Rigid Wheels and Pneumatic Tyres on Yielding Soils With Regard to Tyre Deformation. Journal of Terramechanics, 6(3):73-98, 1969.

[33] MathWorks. MATLAB Version 8.0, Computer Program, 2012.

[34] R. Matsuzaki and A. Todoroki. Wireless Monitoring of Automobile Tires for Intelligent Tires. Sensors, 8(12):8123-8138, Dec. 2008.

[35] Measurement Computing Corporation. PMD-1208LS USB-based Analog and Digital I/O Module User's Guide, Measurement Computing Corporation, 2005.

[36] Measurement Computing Corporation. Data Acquisition Handbook. Norton, MA, 3rd edition, 2012.

[37] Measurement Specialties Inc. Piezo Film Sensors Technical Manual. Rev E, 2008.

[38] Measurement Specialties Inc. Piezo Film Product Guide and Price List, 2012.

[39] G. Meirion-Griffith and M. Spenko. An Empirical Study of the Terramechanics of Small Unmanned Ground Vehicles. In IEEE Aerospace Conference, pages 1-6. IEEE, Mar. 2010. 
[40] A. Meitzler, H. Tiersten, A. Warner, F. Welsh III, D. Berlincourt, and G. Coquin. IEEE Standard on Piezoelectricity, Standards Committee of the IEEE Ultrasonics, Ferroelectrics and Frequency Control Society, 1988.

[41] Moog Components Group. Slip Rings With Through-Bores, Datasheet, 2012. URL http://www.moog.com/products/slip-rings/ commercial-industrial-slip-rings/slip-rings-with-through-bores/ sra-73683/.

[42] K. S. Moon, H. Liang, J. Yi, and B. Mika. Tire Tread Deformation Sensor and Energy Harvester Development for "Smart Tire" Applications. SPIE Proceedings of Sensors and Smart Structures Technologies for Civil, Mechanical, and Aerospace Systems, 6529 (65290K):1-12, Apr. 2007.

[43] T. Muro and J. O'Brien. Terramechanics. Swets \& Zeitlinger B.V., Lisse, The Netherlands, 2004.

[44] K. Nagatani, A. Ikeda, K. Sato, and K. Yoshida. Accurate Estimation of Drawbar Pull of Wheeled Mobile Robots Traversing Sandy Terrain Using Built-in Force Sensor Array Wheel. In Intelligent Robots and Systems (IROS). IEEE/RSJ International Conference on Intelligent Robots and Systems, pages 2373-2378, St. Louis, MO, Oct. 2009.

[45] National Instruments. LabView 2012, Computer Program, 2012.

[46] Northern Tool and Equipment. Knobby Tire on Wheel, 2013. URL http://www . northerntool.com/shop/tools/product_18845_18845.

[47] O. Oflaz. US Patent 7,343,787 B2. Piezoelectric Tire Sensor and Method, 2008.

[48] L. Ojeda. Terrain Trafficability Characterization With a Mobile Robot. In Proceedings of SPIE, volume 5804, pages 235-243. SPIE, 2005.

[49] L. Ojeda and J. Borenstein. Terrain Characterization and Classification With a Mobile Robot. Journal of Field Robotics, 23(2):103-122, 2006.

[50] E. J. Pinto. A Three Dimensional Discretized Tire Model for Soft Soil Applications. Master's thesis, Virginia Tech, Blacksburg, Va, 2012.

[51] A. Pohl and F. Seifert. Wirelessly Interrogable Surface Acoustic Wave Sensors For Vehicular Applications. IEEE Transactions on Instrumentation and Measurement, 46 (4):1031-1038, 1997.

[52] R. Raper and A. Bailey. Inflation Pressure and Dynamic Load Effects on Soil Deformation and Soil-tire Interface Stresses. Transactions of the ASAE, 38(3):685-689, 1995. 
[53] W. Rasband. ImageJ, Computer Program. U. S. National Institutes of Health, 2012. URL http://imagej.nih.gov/ij/.

[54] L. R. Ray, D. C. Brande, and J. H. Lever. Estimation of Net Traction for DifferentialSteered Wheeled Robots. Journal of Terramechanics, 46(3):75-87, 2009.

[55] Roboteq Inc. SDC2130/SDC2150 Motor Controller Datasheet, Datasheet, 2012. URL http://roboteq.com/brushed-dc-motor-controllers/ sdc2130-sdc2150-dual-20a-brushed-dc-motor-controller.

[56] J. T. Roth. Measurement of Soil-Tire Interface Stresses. Master's thesis, Iowa State University, 2010.

[57] T. Ruscher. Personal Interview, 2013.

[58] H. Schwanghart. Measurement of Contact Area, Contact Pressure and Compaction Under Tires in Soft Soil. Journal of Terramechanics, 28(4):309-318, 1991.

[59] Shayang Ye Industrial Co. Ltd. Magnetic Encoders, Datasheet, 2010.

[60] Shayang Ye Industrial Co. Ltd. IG42GM 01\&02 Type, Datasheet, 2011.

[61] A. Shirinov and W. Schomburg. Pressure sensor from a PVDF film. Sensors and Actuators A: Physical, 142(1):48-55, Mar. 2008.

[62] J. Sirohi and I. Chorpa. Fundamental Understanding of Piezoelectric Strain Sensors. Journal of Intelligent Material Systems and Structures, 11(4):246-257, Apr. 2000.

[63] SuperDroid Robots Inc. Heavy Duty 4WD All Terrain Mobile Robot Platform, 2012. URL http://www . superdroidrobots . com/shop/item . aspx?itemid=1477.

[64] D. Swanson. Signal Processing for Intelligent Sensor Systems, volume 4 of Signal Processing and Communications. Marcel Dekker, New York, NY, 1 edition, Apr. 2000.

[65] B. Taylor. Experimental Evaluation and Semi-Empirical Modeling of the Tractive Performance of Rigid and Flexible Wheels on Lunar Soil Simulant. Master's thesis, Virginia Tech, Blacksburg, Va, 2009.

[66] Texas Instruments. MicroSIZE, Single-Supply CMOS Operational Amplifiers MicroAmplifier Series, Datasheet, 2005.

[67] Texas Instruments. High-Speed, Single-Supply, Rail-to-Rail Operational Amplifiers. MicroAmplifier Series, Datasheet, 2012.

[68] Texas Instruments. Amplifiers and Linear, 2013. URL http://www.ti.com/lsds/ti/ analog/amplifiersandlinears/documents.page. 
[69] S. Trolier-McKinstry and P. Muralt. Thin Film Piezoelectrics for MEMS. Journal of Electroceramics, 12(1/2):7-17, Jan. 2004.

[70] A. Tuononen. On-Board Estimation of Dynamic Tyre Forces From Optically Measured Tyre Carcass Deflections. International Journal of Heavy Vehicle Systems, 16(3):362, 2009.

[71] J. Y. Wong. Theory of Ground Vehicles. John Wiley \& Sons, Inc., Hoboken, NJ, 4 edition, 2008.

[72] J. Yi. A Piezo-Sensor-Based Smart Tire System for Mobile Robots and Vehicles. IEEE/ASME Transactions on Mechatronics, 13(1):95-103, Feb. 2008.

[73] J. Yi and H. Liang. A PVDF-Based Deformation and Motion Sensor: Modeling and Experiments. IEEE Sensors Journal, 8(4):384-391, Apr. 2008.

[74] O. Yilmazoglu, M. Brandt, J. Sigmund, E. Genc, and H. L. Hartnagel. Integrated InAs/GaSb 3D Magnetic Field Sensors For "The Intelligent Tire". Sensors and Actuators, 94:59-63, 2001.

[75] R. N. Yong and M. A. Foda. Tribology Model for Determination of Shear Stress Distribution Along the Tyre-Soil Interface. Journal of Terramechanics, 27(2):93-114, 1990.

[76] M. E. Yüksekkaya, W. Oxenham, and M. Tercan. Analysis of Mechanical and Electrical Noise Interfacing the Instrument During Data Acquisition for Measurement of Surface Properties of Textile Fibers. 57(12):2885-2890, 2008. 